Florida International University FIU Digital Commons

11-12-2009

\title{
Effective Reconfigurable Antenna Designs to Enhance Performance and Enable Wireless Powering
}

Shishir S. Punjala

Florida International University, spunj001@fiu.edu

DOI: $10.25148 /$ etd.FI09120829

Follow this and additional works at: https://digitalcommons.fiu.edu/etd

Part of the Electromagnetics and Photonics Commons, and the Other Electrical and Computer Engineering Commons

\section{Recommended Citation}

Punjala, Shishir S., "Effective Reconfigurable Antenna Designs to Enhance Performance and Enable Wireless Powering" (2009). FIU Electronic Theses and Dissertations. 108.

https://digitalcommons.fiu.edu/etd/108 


\title{
FLORIDA INTERNATIONAL UNIVERSITY
}

Miami, Florida

\section{EFFECTIVE RECONFIGURABLE ANTENNA DESIGNS TO ENHANCE} PERFORMANCE AND ENABLE WIRELESS POWERING

A dissertation submitted in partial fulfillment of the

\author{
requirements for the degree of \\ DOCTOR OF PHILOSOPHY \\ in \\ ELECTRICAL ENGINEERING \\ by
}

Shishir Shanker Punjala 


\section{To: Dean Amir Mirmiran}

College of Engineering and Computing

This dissertation, written by Shishir Shanker Punjala, and entitled Effective Reconfigurable Antenna Designs to Enhance Performance and Enable Wireless Powering, having been approved in respect to style and intellectual content, is referred to you for judgment.

We have read this dissertation and recommend that it be approved.

Kia Makki

Syed Ahmed

Deng Pan

Kang Yen

Niki Pissinou, Major Professor

Date of Defense: November 12, 2009

This dissertation of Shishir Shanker Punjala is approved.

Dean Amir Mirmiran

College of Engineering and Computing

Dean George Walker University Graduate School

Florida International University, 2009 
C Copyright 2009 by Shishir Shanker Punjala

All rights reserved. 


\section{DEDICATION}

To my father, mother and sister 


\section{ACKNOWLEDGMENTS}

I would like to express my gratitude to the Telecommunications and Information Technology Institute at FIU for providing me with a family oriented environment that made this research possible. I am indebted to all the faculty members and researchers, who have supported, helped, encouraged, and advised me throughout the course of my research.

This dissertation would not have been into existence if it was not for my advisor Prof. Niki Pissinou, who has not only mended my misguided approach to research, but also served as a role model for me to live a successful and happy life as a researcher. She made sure that I am always focused and never deviate from the objectives of research. I would like to specially thank Dr. Kia Makki for his guidance, support and encouragement. If it hadn't been for Dr. Makki's advice at critical junctures of my career, I would have never crossed over from being a Masters student to a PHD student. My special thanks to Dr. Kang Yen, Dr. Deng Pan and Dr. Syed M. Ahmad who have taken out their valuable time to evaluate my dissertation.

Without my mother's love and guidance and my sister's support, I would never have been able to finish this dissertation. 


\section{ABSTRACT OF THE DISSERTATION \\ EFFECTIVE RECONFIGURABLE ANTENNA DESIGNS TO ENHANCE PERFORMANCE AND ENABLE WIRELESS POWERING \\ by}

\section{Shishir Shanker Punjala}

Florida International University, 2009

Miami, Florida

Professor Niki Pissinou, Major Professor

With the increase in traffic on the internet, there is a greater demand for wireless mobile and ubiquitous applications. These applications need antennas that are not only broadband, but can also work in different frequency spectrums. Even though there is a greater demand for such applications, it is still imperative to conserve power. Thus, there is a need to design multi-broadband antennas that do not use a lot of power. Reconfigurable antennas can work in different frequency spectrums as well as conserve power. The current designs of reconfigurable antennas work only in one band. There is a need to design reconfigurable antennas that work in different frequency spectrums. In this current era of high power consumption there is also a greater demand for wireless powering. This dissertation explores ideal designs of reconfigurable antennas that can improve performance and enable wireless powering. This dissertation also presents lab results of the multi-broadband reconfigurable antenna that was created. A detailed mathematical analyses, as well as extensive simulation results are also presented. The novel reconfigurable antenna designs can be extended to Multiple Input Multiple Output (MIMO) environments and military applications. 


\section{TABLE OF CONTENTS}

\section{CHAPTER}

1 Introduction 1

1.1 Background

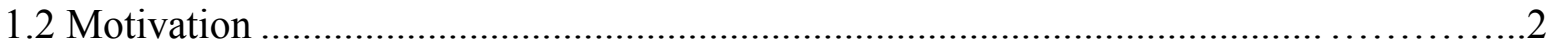

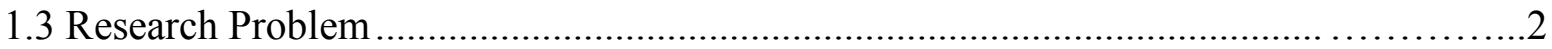

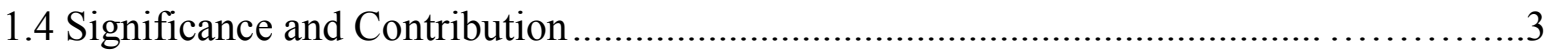

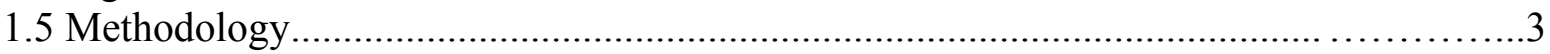

1.6 Organization of the Dissertation...................................................................

2 Related Work

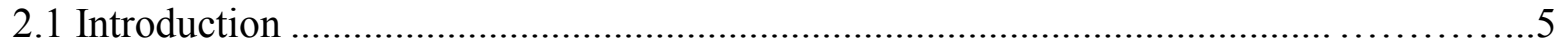

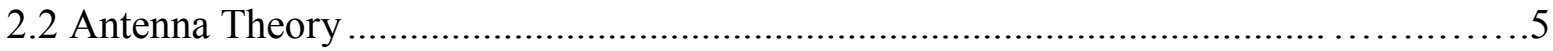

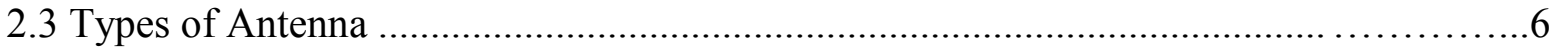

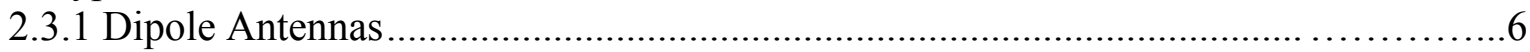

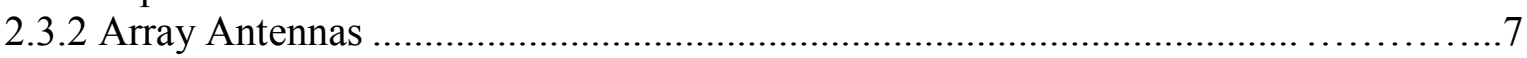

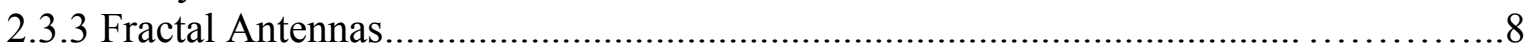

2.3.4 Microstrip Antennas ..............................................................................

2.3.5 Microstrip Fractal Patch Antennas .................................................................

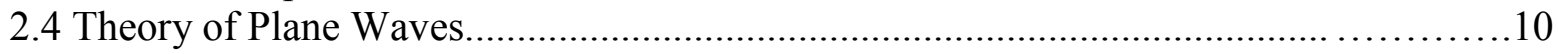

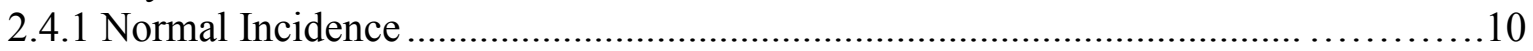

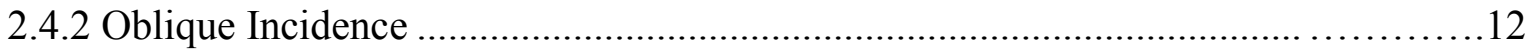

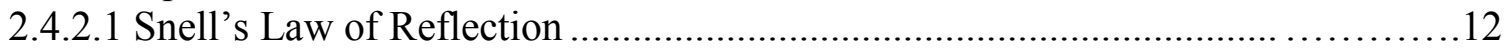

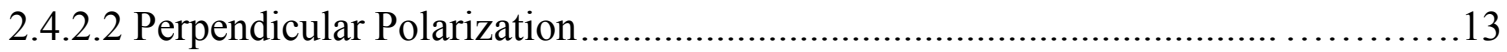

2.4.2.3 Parallel Polarization........................................................................... 14

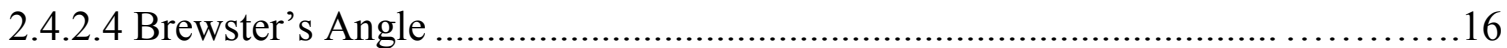

2.4.2.4.1 Perpendicular Polarization .................................................................. 16

2.4.2.4.2 Parallel Polarization ............................................................................ 16

2.5 Permittivity of Concrete ................................................................................. 17

2.6 Radio Frequency Spectrum ................................................................................. 19

2.6.1 Extremely Low and Very Low Frequencies (ELF \& VLF) $(<30 \mathrm{KHz}) \quad \ldots \ldots \ldots \ldots . . .19$

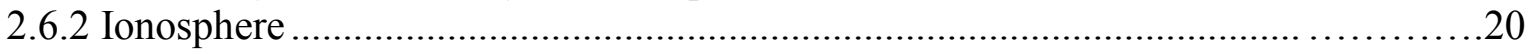

2.6.3 Low and Medium Frequencies (LF \& MF) $(30 \mathrm{KHz}$ to $3 \mathrm{MHz}) \ldots \ldots \ldots \ldots \ldots \ldots \ldots \ldots . . . \ldots . . . . . .20$

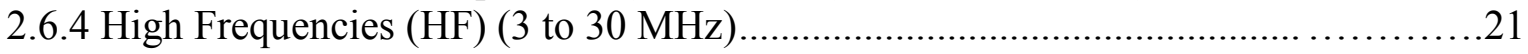

2.6.5 Very High Frequencies and Ultrahigh Frequencies (VHF \& UHF) (30 MHz

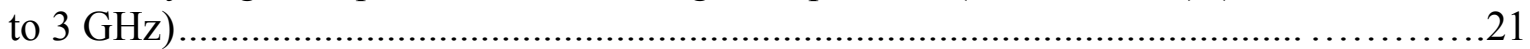

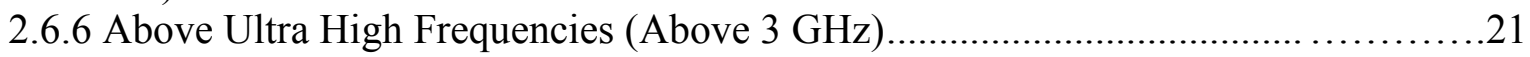

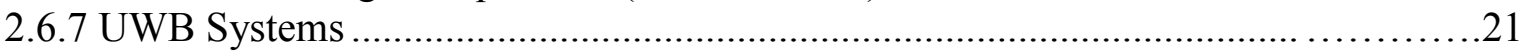

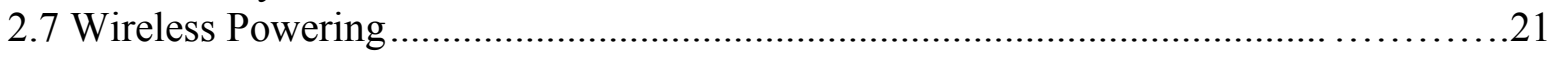

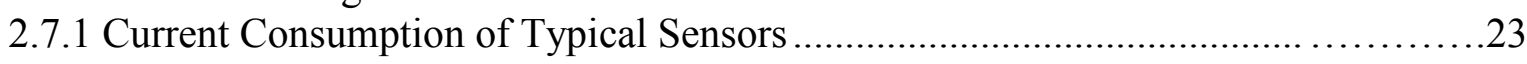

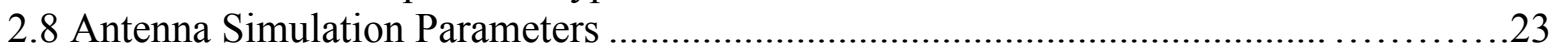

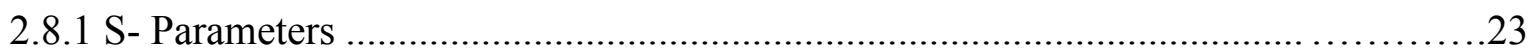

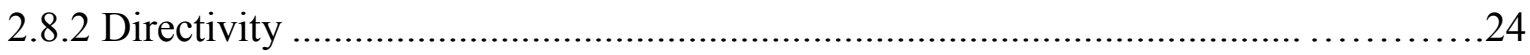

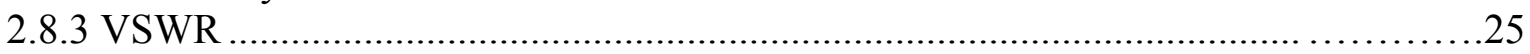

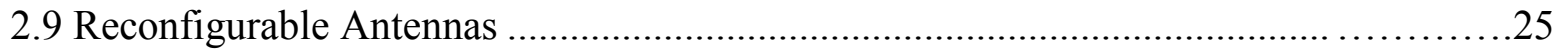

2.9.1 Reconfigurable Sierpinski Gasket Antenna ...................................................26 
2.9.1.1 Reworked Results of the Reconfigurable Sierpinski Gasket Antenna ...........27

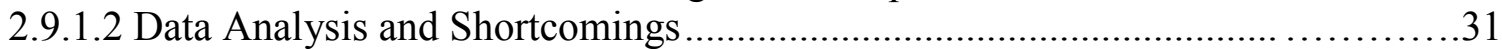

2.9.2 Reconfigurable Planar Inverted Fractal Antenna (RPIFA) .................................

2.9.2.1 Reworked Results of the Reconfigurable Planar Inverted Fractal Antenna

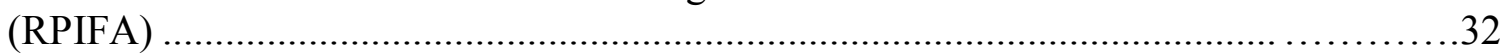

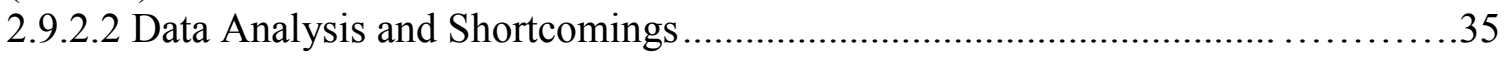

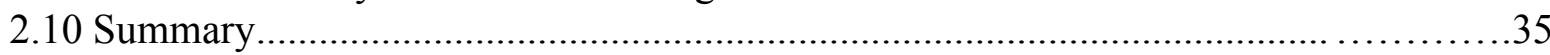

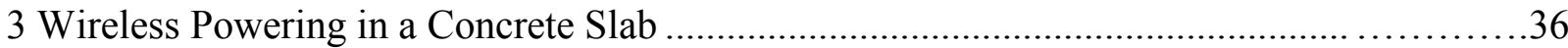

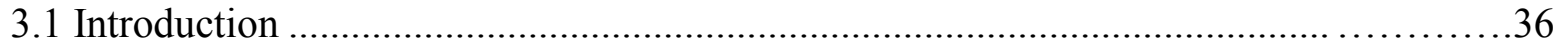

3.2 Uniform Plane Waves in a Concrete Medium-Principal Axis and Oblique

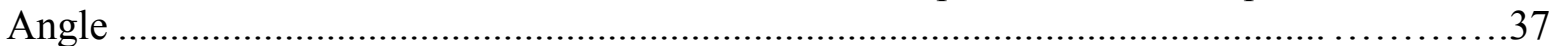

3.2.1 Direction of Propagation of a Uniform Plane Wave ........................................... 38

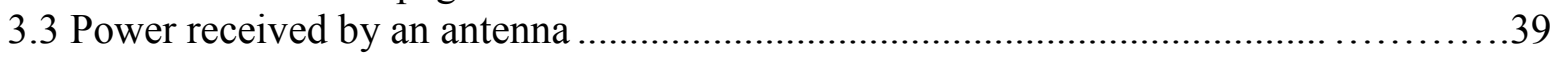

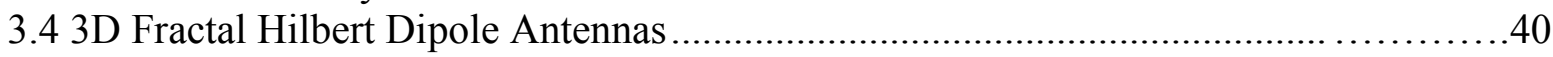

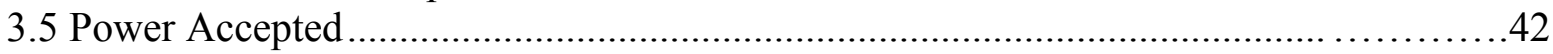

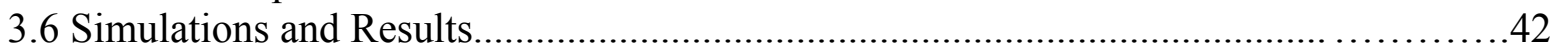

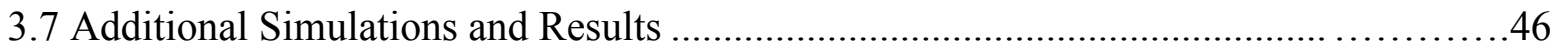

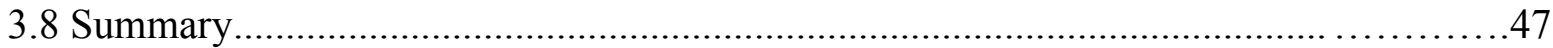

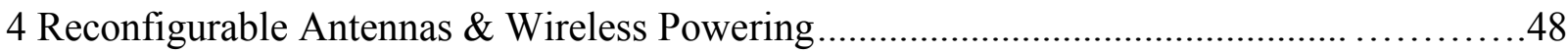

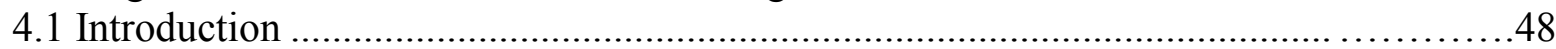

4.2 Reconfigurable Antennas vs. Dipoles for Wireless Powering ................................48

4.3 Reconfigurable Planar Inverted Sierpinski Gasket Fractal

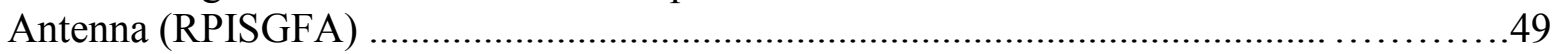

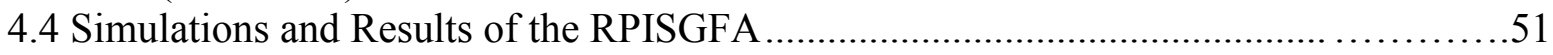

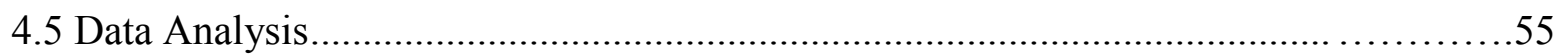

4.6 Simulations and Results of the RPISGFA in a concrete slab .............................55

4.7 Additional Simulations and Results of the RPISGFA in a concrete slab..................57

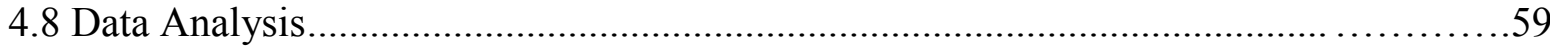

4.9 Simulations and Results of the Planar Reconfigurable Inverted Fractal

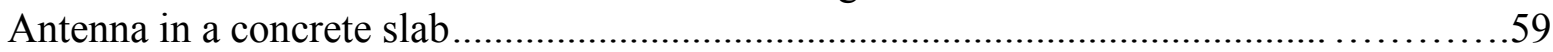

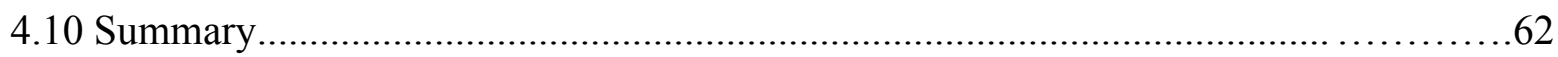

5 Rectangular Reconfigurable Antenna (RRA) with Ultra Wideband Tuning

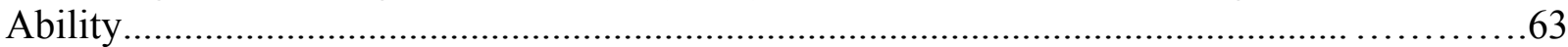

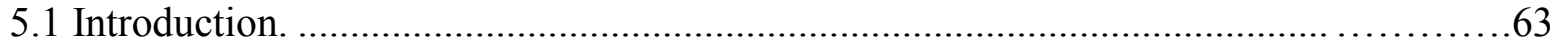

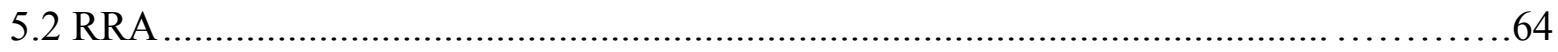

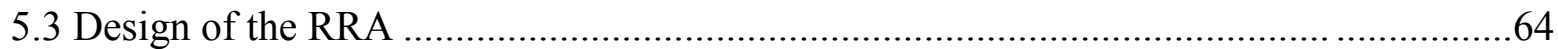

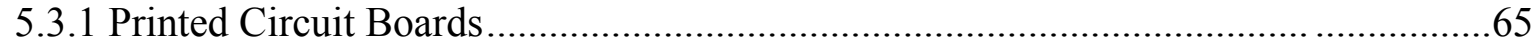

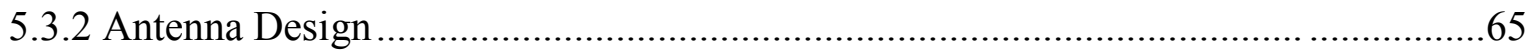

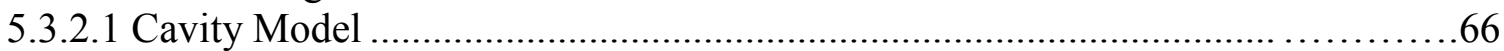

5.3.2.1.1 Field Configurations (Modes) - $\mathrm{TM}^{\mathrm{x}}$..................................................67

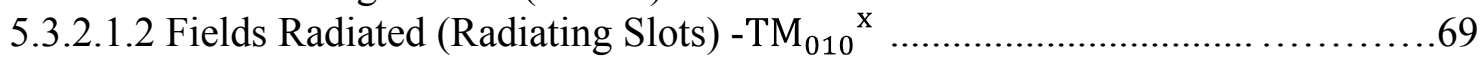

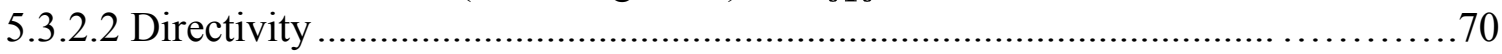

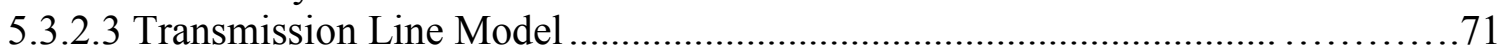

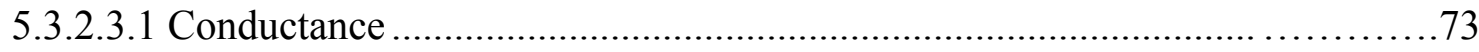

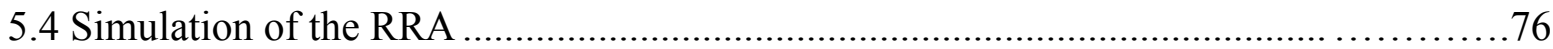




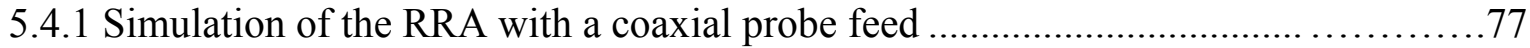

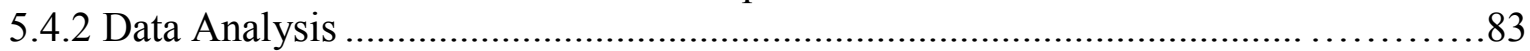

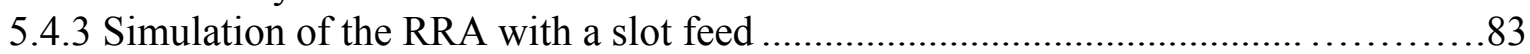

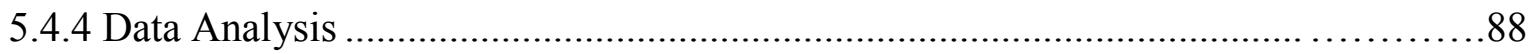

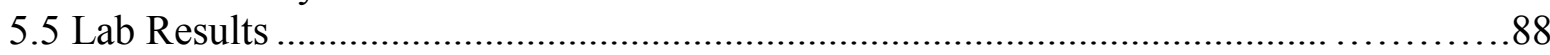

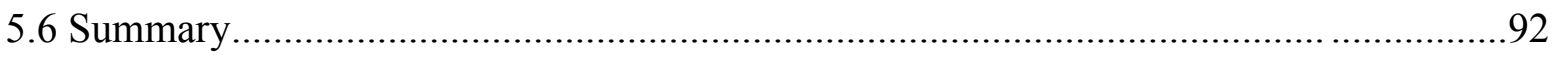

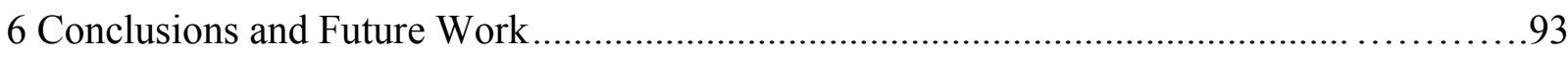

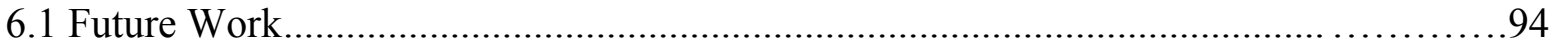

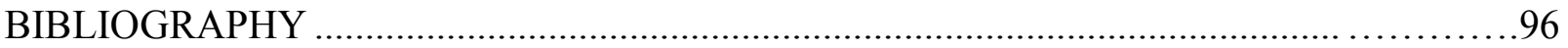

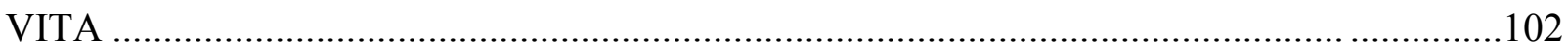


FIGURE

1.1 Radiation Pattern of a Dipole 1

2.1 Transmission System. 6

2.2 Dipole Antenna. .6

2.3 Array Antenna .7

2.4 Fractal Antenna 8

2.5 Microstrip Antenna..

2.6 Microstrip Antenna with fractal geometries. .10

2.7 Plane Wave reflection and transmission for normal incidence

2.8 Plane Wave reflection and transmission for perpendicular polarization

2.9 Plane Wave reflection and transmission for parallel polarization.

2.10 Relative Permittivity of Concrete for the first Debye Model .18

2.11 Relative Permittivity of Concrete for the second Debye Model

2.12 Wireless Power Transmission

2.13 Reconfigurable Sierpinski Gasket Antenna .26

2.14 Reconfigurable Sierpinski Gasket Antenna with iterations .26

2.15 Simulated Reconfigurable Sierpinski Gasket Antenna .27

2.16 Input Return Loss $\left(S_{11}\right)$ for the first iteration .28

2.17 Input Return Loss $\left(\mathrm{S}_{11}\right)$ for the second iteration .28 
2.21 Radiation Pattern for the second iteration

2.24 Input Return Loss $\left(S_{11}\right)$ at lumped port 2.

2.25 VSWR at lumped port 1

2.26 VSWR at lumped port 2

2.27 Radiation Pattern for the Reconfigurable PIFA Antenna.

3.1 Incident Electric Field on a Concrete Slab

3.2 3D Fractal Hilbert Dipole Antenna [18]

3.3 Input Return Loss $\mathrm{S}_{11}(\mathrm{~dB})$ of the surface of the Concrete slice.

3.4 Electric Field inside a concrete slice $(0.2 \%)$ for the incident electric field having $\mathrm{X}$ and $\mathrm{Y}$ components

3.5 Electric Field inside a concrete slice (12\%) for the incident electric field having $\mathrm{X}$ and $\mathrm{Y}$ components

3.6 Electric Field inside a concrete slice (12\%) for the incident electric field having an $\mathrm{X}$ component.

3.7 Electric Field inside a concrete slice $(0.2 \%)$ for the incident electric field having an $\mathrm{X}$ component.

3.8 Power received by a Hilbert 3D-2 Antenna for the First Debye model of relative permittivity of concrete 
3.10 Electric Field inside the concrete slice

4.3 Input Return Loss $\left(\mathrm{S}_{11}\right)$ for the second iteration

4.4 Radiation Pattern for the first iteration .53

4.5 Radiation Pattern for the second iteration .53

4.6 VSWR for the first iteration

4.7 VSWR for the second iteration

4.8 Input Return Loss $\left(\mathrm{S}_{11}\right)$ for a concrete slab ( $0.2 \%$ moisture content $)$, having the RPISGFA $12 \mathrm{~cm}$ inside it

4.9 Input Return Loss $\left(S_{11}\right)$ for a concrete slab (12\% moisture content), having the RPISGFA $12 \mathrm{~cm}$ inside it

4.10 Electric fields on the surface of the RPISGFA buried inside a concrete slab having $0.2 \%$ moisture content .56

4.11 Electric fields on the surface of the RPISGFA buried inside a concrete slab having $12 \%$ moisture content.

4.12 Input Return Loss $\left(S_{11}\right)$ for a concrete slab ( $0.2 \%$ moisture content), having the RPISGFA $10 \mathrm{~cm}$ inside it

4.13 Input Return Loss $\left(S_{11}\right)$ for a concrete slab (12\% moisture content), having the RPISGFA $10 \mathrm{~cm}$ inside it

4.14 Electric field on the surface of the RPISGFA buried inside a concrete slab having $0.2 \%$ moisture content.

4.15 Electric field on the surface of the RPISGFA buried inside a concrete slab having $12 \%$ moisture content 
4.16 Electric field on the surface of the Reconfigurable PIFA antenna buried inside a concrete slab having $0.2 \%$ moisture content

4.17 Electric field on the switches of the Reconfigurable PIFA antenna

buried inside a concrete slab having $0.2 \%$ moisture content

4.18 Electric field on the surface of the Reconfigurable PIFA antenna

buried inside a concrete slab having $12 \%$ moisture content

4.19 Electric field on the switches of the Reconfigurable PIFA antenna

buried inside a concrete slab having $12 \%$ moisture content

5.1 RRA

5.2 PCB

5.3 A Typical Antenna Design .65

$5.4 \mathrm{TM}^{\mathrm{x}}{ }_{010}$ of a rectangular patch antenna

5.5 Effective and physical lengths of a rectangular patch antenna [13]

5.6 Rectangular Patch with its equivalent circuit transmission model

5.7 Equivalent circuit transmission model of the RRA

5.8 Analysis of the Equivalent circuit transmission model of the RRA

5.9 Input Return Losses when the antenna was simulated using an

RT Durroid 5880 substrate for the third iteration.

5.10 Input Return Losses when the antenna was simulated using an FR4 epoxy substrate for the third iteration .78

5.11 Radiation Pattern when the antenna was simulated using an

RT Durroid 5880 substrate for the third iteration

5.12 Radiation Pattern when the antenna was simulated using an

FR4 epoxy substrate for the third iteration .79 
5.13 VSWR when the antenna was simulated using an RT Durroid 5880

substrate for the third iteration

5.14 VSWR when the antenna was simulated using an FR4 epoxy

substrate for the third iteration

5.15 Radiation Pattern when the antenna was simulated using an

RT Durroid 5880 substrate for the second iteration

5.16 Radiation Pattern when the antenna was simulated using an FR4 epoxy

substrate for the second iteration

5.17 VSWR when the antenna was simulated using an RT Durroid 5880

substrate for the second iteration.

5.18 VSWR when the antenna was simulated using an FR4 epoxy substrate

for the second Iteration

5.19 Input Return Losses when the antenna was simulated using an

RT Durroid 5880 substrate for the third iteration

5.20 Input Return Losses when the antenna was simulated using an FR4 epoxy

substrate for the third iteration

5.21 Radiation Pattern when the antenna was simulated using an

RT Durroid 5880 substrate for the third iteration

5.22 Radiation Pattern when the antenna was simulated using an FR4 epoxy

substrate for the third iteration

5.23 VSWR when the antenna was simulated using an RT Durroid 5880

substrate for the third iteration

5.24 VSWR when the antenna was simulated using an FR4 epoxy substrate

for the third iteration .86

5.25 Radiation Pattern when the antenna was simulated using an RT Durroid 5880 substrate for the second iteration

5.26 Radiation Pattern when the antenna was simulated using an FR4 epoxy substrate for the second iteration. 
5.27 VSWR when the antenna was simulated using an RT Durroid 5880 substrate for the second iteration

5.28 VSWR when the antenna was simulated using an FR4 epoxy substrate for the second iteration

5.29 Input Return Losses from the Network Analyser-1

5.30 Input Return Losses from the Network Analyser-2

5.31 Input Return Losses from the Network Analyser-3 .90

5.32 Input Return Losses from the Network Analyser-4 .90

5.33 Input Return Losses from the Network Analyser-5 91

5.34 VSWR from the Network Analyser .91 


\section{LIST OF ACRONYMS}

ACRONYM

PAGE

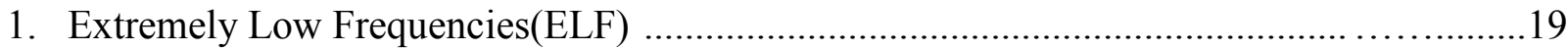

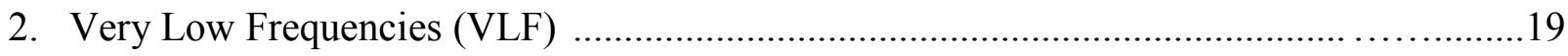

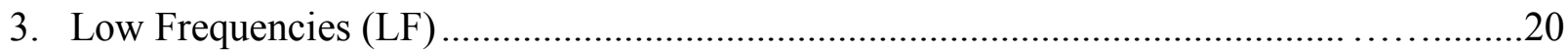

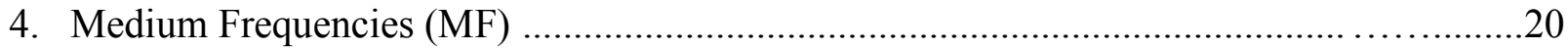

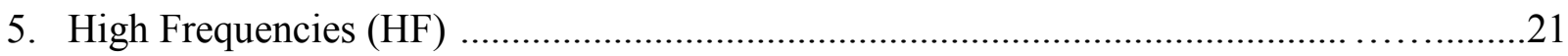

6. Very High Frequencies and Ultrahigh Frequencies (VHF \& UHF) ..............................21

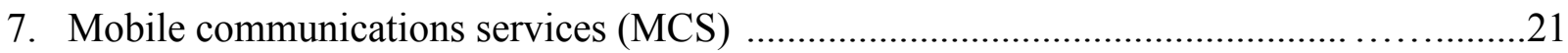

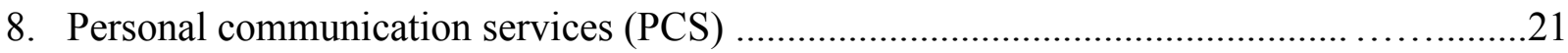

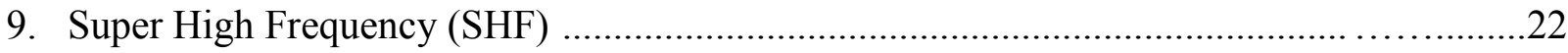

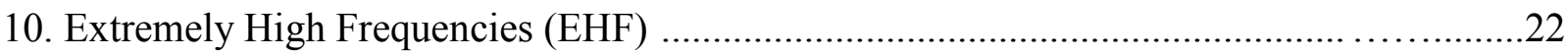

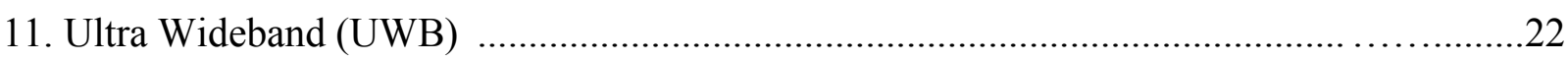

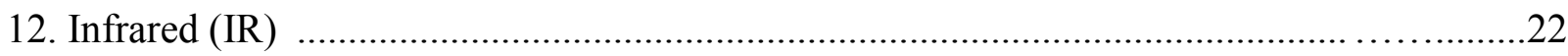

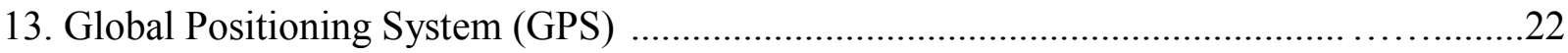

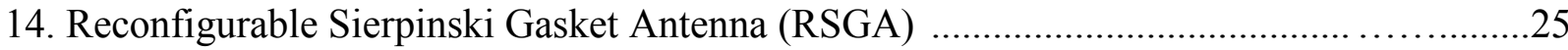

15. Reconfigurable Planar Inverted Fractal Antenna (RPIFA) ..........................................31

16. Reconfigurable Planar Inverted Sierpinski Gasket Fractal Antenna (RPISGFA) .48

17. Rectangular Reconfigurable Antenna (RRA). .63 


\section{Chapter 1}

\section{Introduction}

Conventional antenna designs such as dipoles and Microstrip antennas [13] do not support multiple radiation patterns and use a lot of power. A patch antenna [13] uses a larger amount of current supply if it wishes to reach out to a farther distance. The radiation pattern of a dipole shown in fig.1.1 can be changed by using software techniques, but the antenna cannot change its radiation pattern by varying its size. Reconfigurable antennas can change their radiation patterns, as well as use the same amount of current supply to improve the quality of received signal at the receiver end. The existing reconfigurable designs are studied and their shortcomings are explained in this dissertation. This research shows that wireless powering in mediums such as a concrete slab is possible. This research improves the existing designs of reconfigurable antennas, as well as shows a new reconfigurable antenna design to enhance the performance of wireless devices and enable wireless powering.

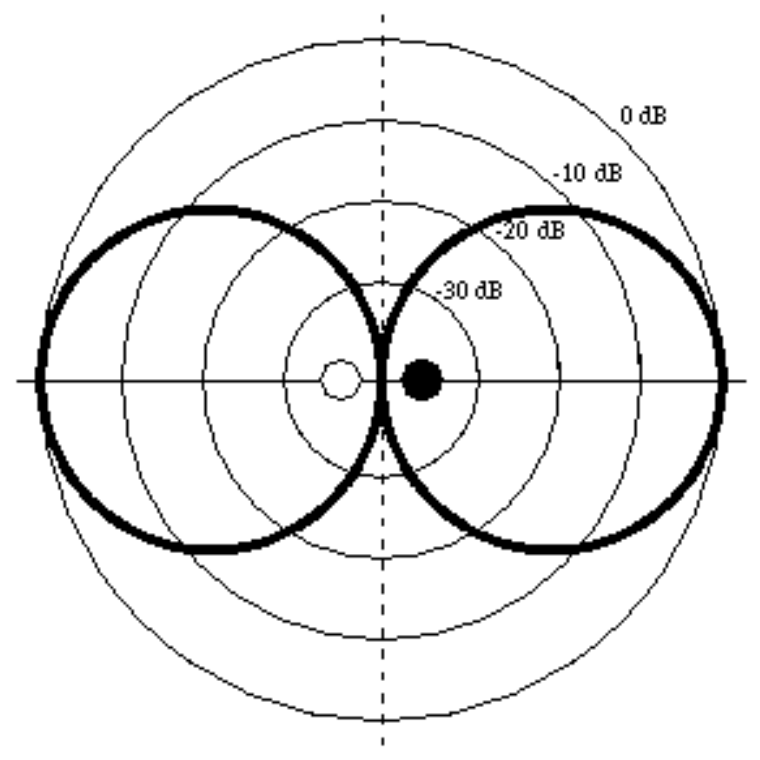

Fig.1.1 Radiation Pattern of a Dipole

\subsection{Background}

Reconfigurable antennas consist of switches and radiators. The radiators can be either planar patches or dipoles. The switches can turn a section of radiators off and thus stop them 
from radiating. By using more radiators, the quality of the received signal can be improved increasing the input power supply. Since dipoles are obsolete, this dissertation focuses only on reconfigurable patch antenna designs.

\subsection{Motivation}

Even though a dipole antenna can be used over a certain range of frequencies, it has only one very good frequency point called the resonating frequency, at which it works best. A reconfigurable antenna can work best at more than three frequencies, if designed optimally. Since a reconfigurable antenna has more resonating frequencies, it can be used to enable more applications. The current antenna designs are larger in size and involve only one feeding point. This research would make the antenna more compact, provide multiple radiation patterns as well as more than one feeder point. The improvements in this dissertation will bring about a successful implementation of the reconfigurable antenna in mobile devices.

Reconfigurable antenna design provides multiple points that can be used to read signals from to take in power. Research in the past has never shown whether wireless powering in a concrete slab is possible. Reconfigurable antennas can be used to power sensors inside a concrete slab. The proposed improvements would not only enhance antenna performance in transmission, but also provide huge advantages in wireless powering.

\subsection{Research Problem}

Improvised designs would enhance portability in mobile devices as well as have higher bandwidth. Many sensors are buried inside a concrete slab, to measure parameters such as temperature and humidity. If these sensors are powered wirelessly, with reconfigurable antennas, then it would be a significant improvement in enabling wireless powering. During the transmission of radio frequency energy from one antenna to another, the electric field could either be incident parallel to the receiving antenna or be incident at a certain angle. This incident electric field on the surface of the receiving antenna can be used to power a sensor 
wirelessly. The research problem has three main goals: studying the existing reconfigurable antenna designs $[1,2]$, recreating them in Ansoft HFSS and improving them, as well as to design a new reconfigurable antenna that enhances performance and enables wireless powering.

\subsection{Significance and Contribution}

Antennas are critical components of communication and radar systems [70], but sometimes their inability to adjust to new operating scenarios can limit system performance. Making antennas reconfigurable [70], so that their behavior can adapt with changing system requirements or environmental conditions can eliminate these restrictions and provide additional levels of functionality. Reconfigurable antennas on portable wireless devices can help to improve a noisy connection and redirect transmitted power to conserve battery life. In large phased arrays, reconfigurable antennas could be used to provide additional capabilities that may result in wider instantaneous frequency bandwidths, more extensive scan volumes, and radiation patterns with more desirable side lobe distributions.

Reconfigurable antennas providing numerous advantages such as reconfigurability in polarization, frequency, and radiation pattern [67]. Furthermore, these antennas can reduce parasitic effects, losses and costs. Reconfigurable antennas have been used to achieve pattern and frequency diversity in Single Input Single Output (SISO) links, and are being used in Multiple Input Multiple Output (MIMO) systems [68, 69] to improve link capacity. New designs of reconfigurable antennas have been shown in this dissertation that can improve performance and enable mobile and ubiquitous computing applications.

\subsection{Methodology}

All simulations of antennas in this dissertation have been done using Ansoft HFSS. Different excitation methods such as a lumped port, waveport and a slot were used to excite the antennas. The concrete slabs were excited using plane waves. The new antenna designs have 
been simulated in Ansoft HFSS v11, and the Rectangular Reconfigurable Antenna was made on a double sided PCB with an RF connector and tested using a network analyzer.

\subsection{Organization of the Dissertation}

This dissertation is organized as follows: In Chapter 2, a comprehensive literature survey of wireless powering, a concrete slab and reconfigurable antennas are presented. The permittivity of a concrete slab and reworked results of two reconfigurable antennas are also presented in Chapter 2. In Chapter 3, a concrete slice was prepared in Ansoft HFSS v8, and it has been shown that wireless powering in a concrete slab is possible using a Hilbert 3D-2 antenna. In Chapter 4, a novel planar reconfigurable antenna design is presented along with the simulation results. The existing reconfigurable antenna designs have been simulated in a concrete slab, and their results are also presented and analyzed in Chapter 4. In Chapter 5, a new reconfigurable antenna that can be created for any frequency range is presented. The design equation of this novel reconfigurable antenna, as well as an extensive mathematical derivation of its electric field, is presented in Chapter 5. The power radiated equation and its directivity are also presented. Chapter 6 concludes the dissertation and summarizes the results of the work. Areas for future work are also suggested in this chapter. 


\section{Chapter 2 \\ Related Work}

\subsection{Introduction}

Communication [52] is defined as a process where there is an exchange of information between two sides. If the exchange of information involves electromagnetic or radio waves, then the process is called radio communication. An antenna is defined $[13,45]$ as a device that either radiates or receives radio waves. Typically, an antenna is excited by using a coaxial line [13] that transports electromagnetic energy from the transmitting unit into the antenna. An antenna system has a radiation pattern, which gives the user an idea of the direction in which the antenna radiates. Software defined radio techniques can be used to change the direction in which an antenna is radiating. Different antenna designs were reworked, and their shortcomings are explained with the results in this chapter.

Fractal antenna theory design [52] is an extension of Euclidian geometry. Fractal antennas [37] are specific types of antennas which consist of multiple copies of a single antenna. Antenna research deals with two core issues- design and its implementation-, to develop multiband smaller size antennas. These fractal shaped designs [38] were used to enhance the existing designs of microstrip antennas, which led to the eventual realization of reconfigurable antennas. Reconfigurable antennas are a specific design of antennas that are used to save power.

\subsection{Antenna Theory}

An antenna is a system of elevated conductors that connect the transmitter or the receiver to free space. In order to understand an antenna system, let us consider fig. 2.1. A transmission line is a point to point radio frequency carrier device with minimum attenuation and radiation losses. The transmission line has to be matched in such a way that a forward 
moving wave travels only in the forward direction. A dipole antenna is a specific type of

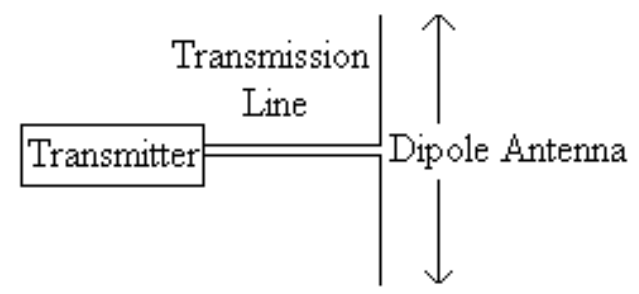

Fig. 2.1 Transmission System

antenna in which the two ends are at equal potential relative to the mid-point, and the antenna itself is fed at the centre by the transmission line. The parameters typically discussed in this chapter include:

- Input Return Loss

- Antenna Far Field

- Directivity Pattern

- Resonating Frequency

- VSWR

\subsection{Types of Antennas}

The various types of antennas that were studied are described below.

\subsubsection{Dipole Antennas}

Developed by Heinrich Rudolph Hertz [54] in the late $19^{\text {th }}$ century, a Dipole is an antenna made by a simple copper wire, and is a center-fed driven element for transmitting or receiving radio frequency energy. The amplitude of current on a dipole antenna decreases uniformly, from maximum at the center to zero at the ends.

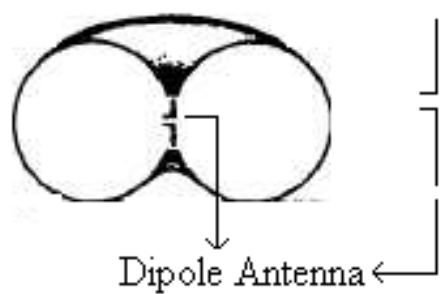

Fig. 2.2 Dipole Antenna 
A dipole shown in fig. 2.2 radiates on both sides and can have any length, but usually it is just under $1 / 2$ wavelengths long. A dipole which is $1 / 2$ wavelengths long is known as a resonant, or a half wave dipole, and has input impedance that is purely resistive and lies between 30 and 80 ohms. A half wave dipole provides a good match to $50 \mathrm{ohms}$ coaxial cables, as well as to transmitters and receivers which have $50 \mathrm{ohm}$ output and input impedances. The length of a dipole can be approximately determined from the following formula

$$
l=\frac{468}{\mathrm{f}}
$$

Where $\mathrm{l}$ is the length in feet, and $\mathrm{f}$ is the frequency in $\mathrm{MHz}$

\subsubsection{Array Antennas}

Many applications require different radiation patterns that cannot be achieved by a single radiating element. An array antenna consists of multiple radiating elements formed in a mathematical or a geometrical arrangement. The radiation pattern of an array antenna is a combined radiation pattern of all the radiating elements. The array antenna shown in fig. 2.3 consists of multiple radiating elements placed on the $\mathrm{z}$ axis with a space $\mathrm{d}$ between them.

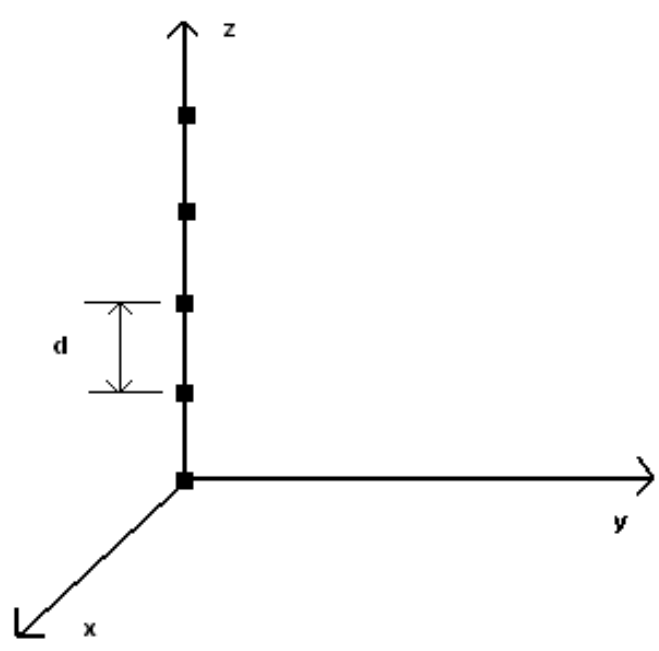

Fig. 2.3 Array Antenna 


\subsubsection{Fractal Antennas}

Fractal electrodynamics [37] is a field of electrodynamics which combines fractal geometry and electromagnetic theory to solve the problems of radiation, propagation, and scattering in antennas. A fractal is a rough or fragmented geometric shape [54] which can be subdivided in parts, each of which is a reduced-size copy of the whole. Fractals are generally self-similar and independent of scale. A case of a log periodic antenna [37] folding inwards is considered to be a fractal antenna. A simple example of a fractal antenna could be an ordinary wire antenna shaped into many similar shapes. The size of the antenna would thus be very large. Since each shape of the fractal antenna is analyzed as a conjunction of capacitors and inductors, the fractal antenna would thus be a combination of multiple capacitors and inductors. It should be understood that the number of resonances would not depend on the size of the radiating structure, but on the combination of capacitors and inductors being used.

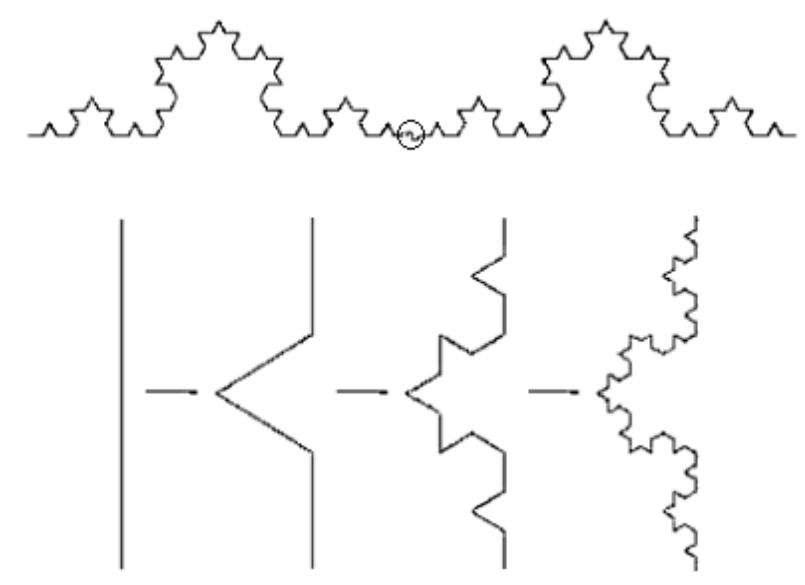

Fig. 2.4 Fractal Antenna

\subsubsection{Microstrip Antennas}

Microstrip antennas, also called patch antennas, consist of a metallic strip placed on a small fraction of a wavelength on a substrate situated on a ground plane. There are many 
substrates [13] that can be used for the design of microstrip antennas, and their dielectric constants are generally in the range of $2.2 \leq \varepsilon_{\mathrm{r}} \leq 12$. Thick substrates with low dielectric constant values provide better efficiency, larger bandwidth and loosely bound fields for radiation into space.

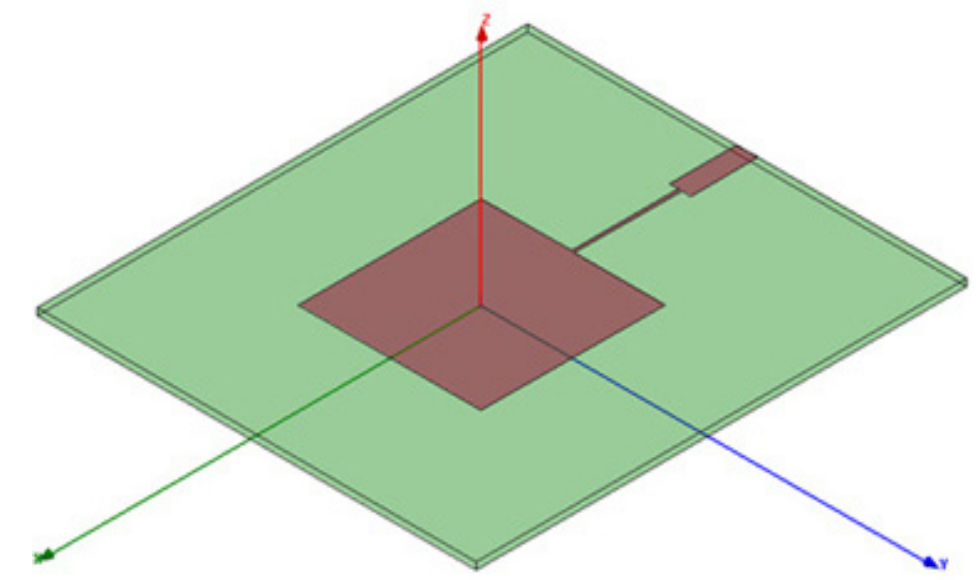

Fig. 2.5 Microstrip Antenna

Microstrip antennas can also be circular, square, elliptical or triangular shaped. Microstrip antennas can be excited by numerous methods. The popular feeding methods used to excite microstrip antennas are microstrip line, coaxial probe, aperture coupling and proximity coupling.

\subsubsection{Microstrip Fractal Patch Antennas}

As stated earlier, fractal antennas are a conjunction of multiple capacitors and inductors. This self similarity concept in fractal antennas provides more than one resonating frequency. This concept of fractal antenna design was extended to microstrip antennas. So a single microstrip fractal antenna consists of many self similar antennas, thus forming a microstrip fractal patch antenna. Microstrip antennas with fractal geometry have been found [2, $38,40]$ to have a higher degree of directivity and multiple resonating frequencies. 


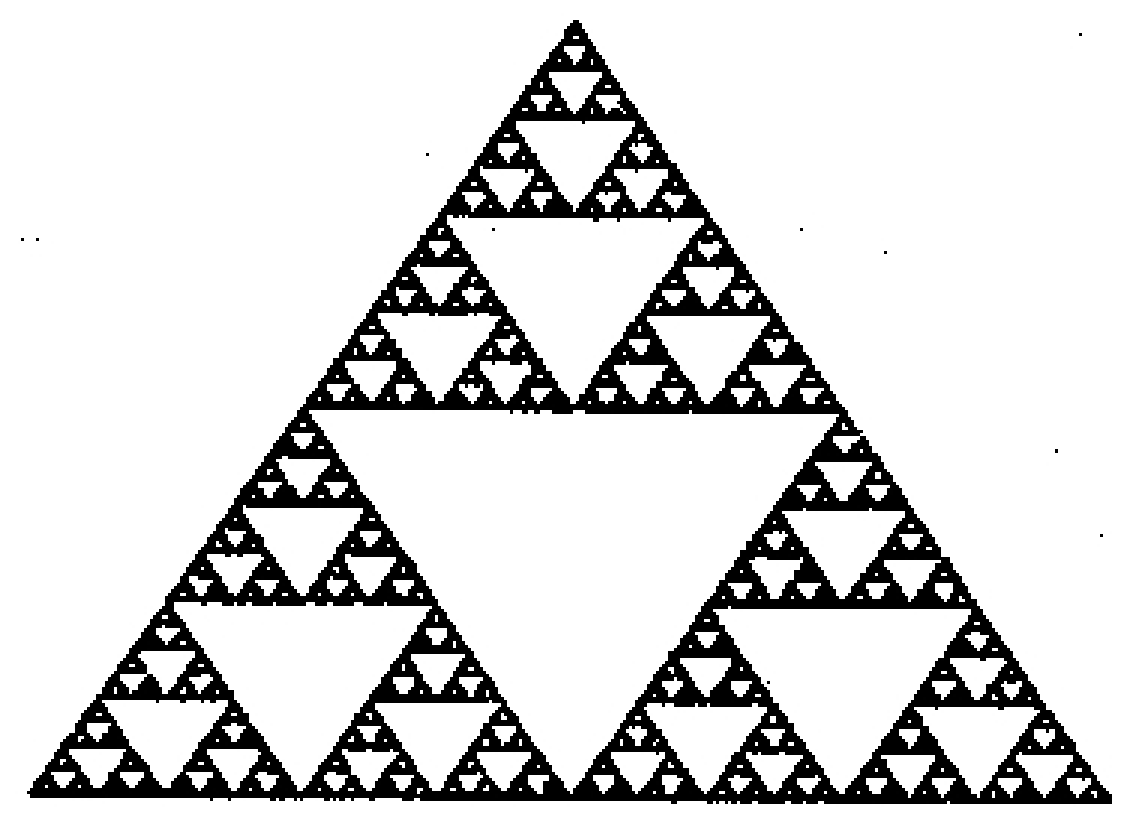

Fig. 2.6 Microstrip Antenna with fractal geometries

\subsection{Theory of Plane Waves}

This research deals with improving the design of reconfigurable antennas to enhance performance, and to enable wireless powering in mediums such as a concrete slab. Thus, it is important to understand the propagation of electric field in other mediums when their surface is excited by a plane wave. The propagation of electric field involves two cases: when the electric field is incident normal to the surface of the medium (normal incidence), and when the electric field is incident perpendicular to the surface of the medium (oblique incidence).

\subsubsection{Normal Incidence}

The reflection and transmission of plane waves when the electric field is incident normal to the surface of the medium $[12,14]$ is discussed first. The incident, reflected and transmitted electric and magnetic fields, are denoted by the subscripts i, $\mathrm{r}$ and $\mathrm{t}$ respectively. Each particular medium has its own parameters of permeability $\mu$ and permittivity $\varepsilon$, as shown in fig. 2.7. 


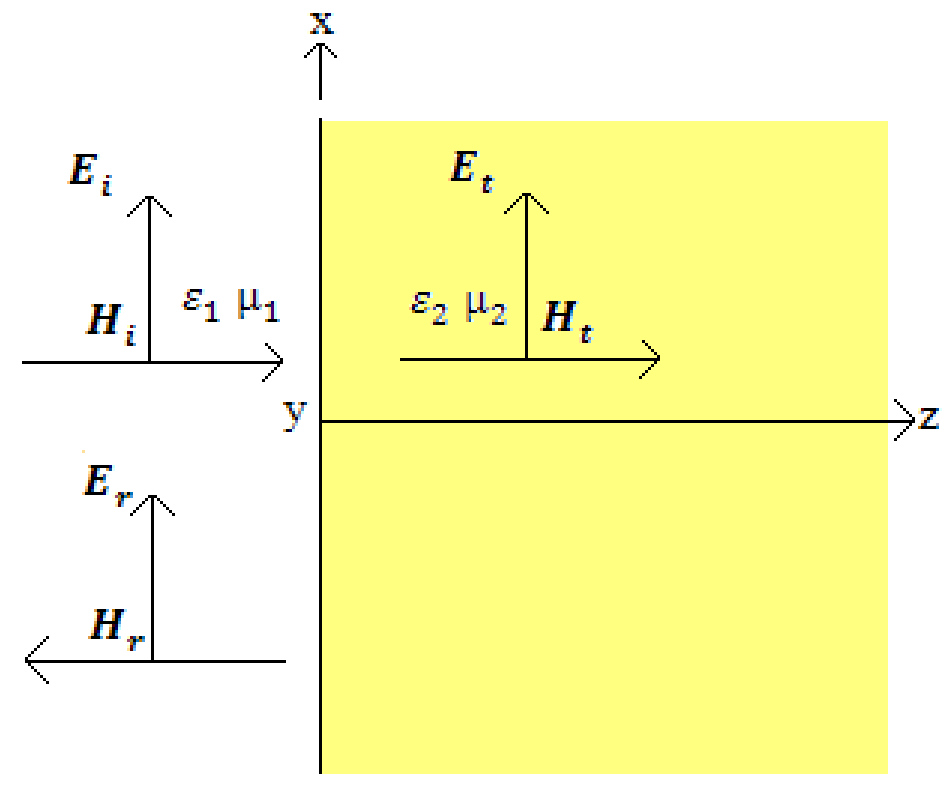

Fig. 2.7 Plane Wave reflection and transmission for normal incidence

If the incident electric field has an amplitude $\mathrm{E}_{0}$, polarized in the $\mathrm{x}$ direction with a component $S_{x}$, then the expressions for the incident, reflected and transmitted electric field components can be written as

$$
\begin{aligned}
& E_{i}=S_{x} E_{0} e^{-j \beta_{1} z} \\
& E_{r}=S_{x} \Gamma^{b} \quad E_{0} e^{+j \beta_{1} z} \\
& E_{t}=S_{x} T^{b} \quad E_{0} e^{-j \beta_{2} z}
\end{aligned}
$$

In the above equations, $\Gamma^{\mathrm{b}}$ and $\mathrm{T}^{\mathrm{b}}$ are the reflection and the transmission coefficients respectively. The magnetic field components can be written as

$$
\begin{gathered}
\mathrm{H}_{\mathrm{i}}=\mathrm{S}_{\mathrm{y}} \frac{\mathrm{E}_{0}}{\eta_{1}} \mathrm{e}^{-\mathrm{j} \beta_{1} \mathrm{z}} \\
\mathrm{H}_{\mathrm{r}}=-\mathrm{S}_{\mathrm{y}} \frac{\Gamma^{\mathrm{b}} \mathrm{E}_{0}}{\eta_{1}} \mathrm{e}^{+\mathrm{j} \beta_{1} \mathrm{z}}
\end{gathered}
$$




$$
\mathrm{H}_{\mathrm{t}}=\mathrm{S}_{\mathrm{y}} \frac{\mathrm{T}^{\mathrm{b}} \mathrm{E}_{0}}{\eta_{1}} \mathrm{e}^{-\mathrm{j} \beta_{2} \mathrm{z}}
$$

Finally, the equations for the reflection and transmission coefficients can be written as

$$
\begin{gathered}
\Gamma^{\mathrm{b}}=\frac{\eta_{2}-\eta_{1}}{\eta_{2}+\eta_{1}}=\frac{\mathrm{E}_{\mathrm{r}}}{\mathrm{E}_{\mathrm{i}}}=-\frac{\mathrm{H}_{\mathrm{r}}}{\mathrm{H}_{\mathrm{i}}} \\
\mathrm{T}^{\mathrm{b}}=\frac{2 \eta_{2}}{\eta_{2}+\eta_{1}}=1+\Gamma^{\mathrm{b}}=\frac{\mathrm{E}_{\mathrm{t}}}{\mathrm{E}_{\mathrm{i}}}=\frac{\eta_{2}}{\eta_{1}} \frac{\mathrm{H}_{\mathrm{t}}}{\mathrm{H}_{\mathrm{i}}}
\end{gathered}
$$

\subsubsection{Oblique Incidence}

A plane is formed by a unit vector perpendicular $[12,14]$ to the reflection interface, and the vector in the direction of incidence. In order to provide more clarity, the electric and magnetic fields would have their own parallel and perpendicular components, and the reflection and the transmission coefficients would also change depending on the polarization of the incident electric field. Perpendicular polarization [14] is also known as horizontal polarization, and parallel polarization is also known as vertical polarization.

\subsubsection{Snell's Law of Reflection}

Snell's Law of Reflection [14] states that the angle of reflection is always equal to the angle of incidence. Snell's Law of Refraction provides a relation between the angle of incidence and the angle of transmission, in terms of the ratio of phase velocities. The ratio of the phase velocity in free space to the phase velocity in the medium is defined as the index of refraction of a medium $n$.

$$
\mathrm{n}=\frac{\mathrm{c}}{\mu_{\mathrm{p}}}=\sqrt{\frac{\mu \epsilon}{\mu_{0} \epsilon_{0}}}=\sqrt{\mu_{\mathrm{r}} \epsilon_{\mathrm{r}}}
$$

Where $\mathrm{c}$ is the velocity of light. 
Snell's law of Refraction can be written as

$$
\frac{\sin \theta_{\mathrm{t}}}{\sin \theta_{\mathrm{i}}}=\frac{\eta_{1}}{\eta_{2}}=\sqrt{\frac{\mu_{\mathrm{r}_{1}} \varepsilon_{\mathrm{r}_{1}}}{\mu_{\mathrm{r}_{2}} \varepsilon_{\mathrm{r}_{2}}}}
$$

The subscript $r$ denotes the relative permeability and relative permittivity.

For nonmagnetic materials, $\mu_{\mathrm{r}_{1}}=\mu_{\mathrm{r}_{2}}=1$, so in this case

$$
\frac{\sin \theta_{\mathrm{t}}}{\sin \theta_{\mathrm{i}}}=\frac{\eta_{1}}{\eta_{2}}=\sqrt{\frac{\varepsilon_{\mathrm{r}_{1}}}{\varepsilon_{\mathrm{r}_{2}}}}=\frac{\eta_{2}}{\eta_{1}}
$$

where $\eta=\sqrt{\frac{\mu}{\varepsilon}}$ is the intrinsic impedance of a dielectric medium.

\subsubsection{Perpendicular Polarization}

In the first case, we assume that the incident electric field is perpendicular [55] to the planar surface as shown in fig. 2.8. The plane wave is incident on the planar surface at an angle, and the equations for the incident, reflected and transmitted electric and magnetic fields can be written as

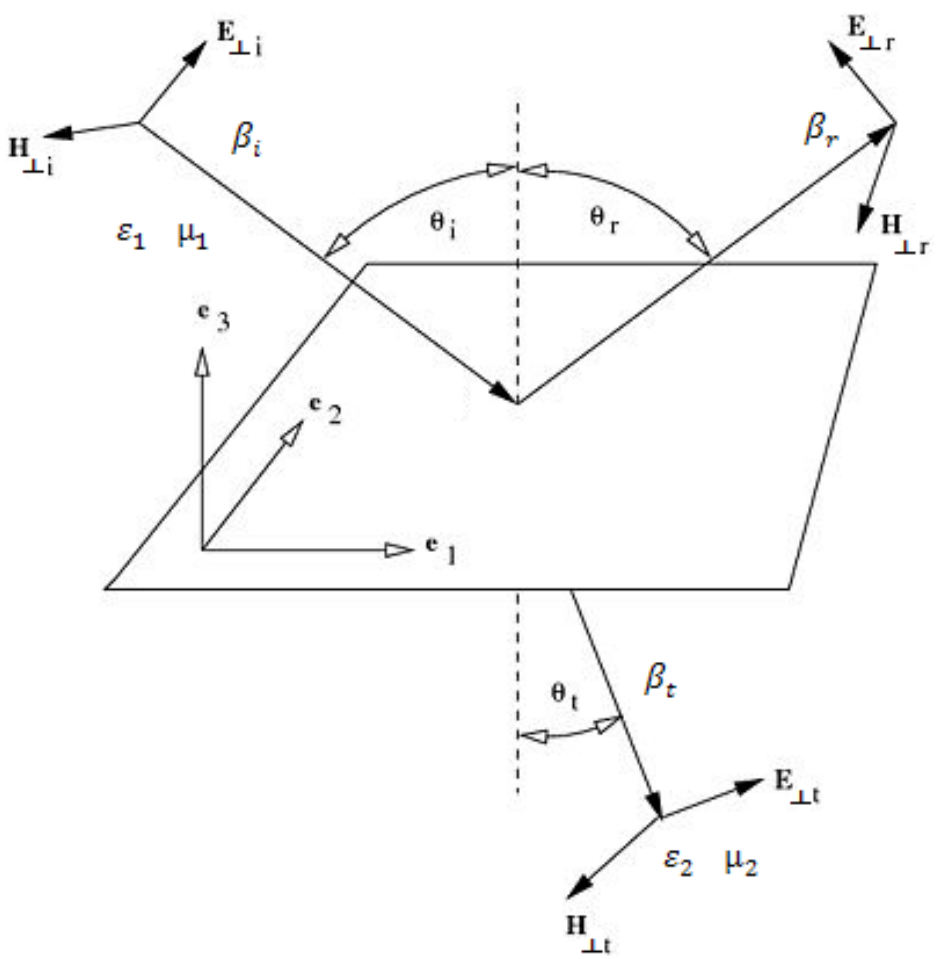

Fig. 2.8 Plane Wave reflection and transmission for perpendicular polarization 


$$
\begin{gathered}
\mathrm{E}_{\perp_{\mathrm{i}}}=\mathrm{S}_{\mathrm{y}} \mathrm{E}_{0} \mathrm{e}^{-\mathrm{j} \beta_{1}\left(\mathrm{x} \sin \theta_{\mathrm{i}}+\mathrm{z} \cos \theta_{\mathrm{i}}\right)} \\
\mathrm{H}_{\perp_{\mathrm{i}}}=\left(-\mathrm{S}_{\mathrm{x}} \cos \theta_{\mathrm{i}}+\mathrm{S}_{\mathrm{z}} \sin \theta_{\mathrm{i}}\right) \frac{\mathrm{E}_{0}}{\eta_{1}} \mathrm{e}^{-\mathrm{j} \beta_{1}\left(\mathrm{x} \sin \theta_{\mathrm{i}}+\mathrm{z} \cos \theta_{\mathrm{i}}\right)} \\
\mathrm{E}_{\perp_{\mathrm{r}}}=\mathrm{S}_{\mathrm{y}} \Gamma_{\perp}{ }^{\mathrm{b}} \mathrm{E}_{0} \mathrm{e}^{-\mathrm{j} \beta_{1}\left(\mathrm{x} \sin \theta_{\mathrm{r}}-\mathrm{z} \cos \theta_{\mathrm{r}}\right)} \\
\mathrm{H}_{\perp_{\mathrm{r}}}=\left(\mathrm{S}_{\mathrm{z}} \sin \theta_{\mathrm{r}}+\mathrm{S}_{\mathrm{x}} \cos \theta_{\mathrm{r}}\right) \frac{\Gamma^{\mathrm{b}} \mathrm{E}_{0}}{\eta_{1}} \mathrm{e}^{-\mathrm{j} \beta_{1}\left(\mathrm{x} \sin \theta_{\mathrm{r}}-\mathrm{z} \cos \theta_{\mathrm{r}}\right)} \\
\mathrm{E}_{\perp_{\mathrm{t}}}=\mathrm{S}_{\mathrm{y}} \mathrm{T}_{\perp}{ }^{\mathrm{b}} \mathrm{E}_{0} \mathrm{e}^{-\mathrm{j} \beta_{2}\left(\mathrm{x} \sin \theta_{\mathrm{t}}+\mathrm{z} \cos \theta_{\mathrm{t}}\right)} \quad(2.17) \\
\mathrm{H}_{\perp_{\mathrm{t}}}=\left(\mathrm{S}_{\mathrm{z}} \sin \theta_{\mathrm{t}}-\mathrm{S}_{\mathrm{x}} \cos \theta_{\mathrm{t}}\right) \frac{\mathrm{T}^{\mathrm{b}} \mathrm{E}_{0}}{\eta_{2}} \mathrm{e}^{-\mathrm{j} \beta_{2}\left(\mathrm{x} \sin \theta_{\mathrm{t}}+\mathrm{z} \cos \theta_{\mathrm{t}}\right)}
\end{gathered}
$$

By using Snell's Law, the equations for the reflection and transmission coefficients can be written as

$$
\begin{gathered}
\Gamma^{\mathrm{b}}=\frac{\eta_{2} \cos \theta_{\mathrm{i}}-\eta_{1} \cos \theta_{\mathrm{t}}}{\eta_{2} \cos \theta_{\mathrm{i}}+\eta_{1} \cos \theta_{\mathrm{t}}}=\frac{\sqrt{\frac{\mu_{2}}{\epsilon_{2}}} \cos \theta_{\mathrm{i}}-\sqrt{\frac{\mu_{1}}{\epsilon_{1}}} \cos \theta_{\mathrm{t}}}{\sqrt{\frac{\mu_{2}}{\epsilon_{2}}} \cos \theta_{\mathrm{i}}+\sqrt{\frac{\mu_{1}}{\epsilon_{1}}} \cos \theta_{\mathrm{t}}} \\
\mathrm{T}^{\mathrm{b}}=\frac{2 \eta_{2} \cos \theta_{\mathrm{i}}}{\eta_{2} \cos \theta_{\mathrm{i}}+\eta_{1} \cos \theta_{\mathrm{t}}}=1+\Gamma^{\mathrm{b}}=\frac{2 \sqrt{\frac{\mu_{2}}{\epsilon_{2}}} \cos \theta_{\mathrm{i}}}{\sqrt{\frac{\mu_{2}}{\epsilon_{2}}} \cos \theta_{\mathrm{i}}+\sqrt{\frac{\mu_{1}}{\epsilon_{1}}} \cos \theta_{\mathrm{t}}}
\end{gathered}
$$

\subsubsection{Parallel Polarization}

In the second case, we assume that the incident electric field is parallel [55] to the planar surface as shown in fig. 2.9. The plane wave is incident on the planar surface at an angle, and the equations for the incident, reflected and transmitted electric and magnetic fields can be written as 


$$
E_{\| i}=\left(S_{x} \cos \theta_{i}-S_{z} \sin \theta_{i}\right) E_{0} e^{-j \beta_{1}\left(x \sin \theta_{i}+z \cos \theta_{i}\right)}
$$

$$
\mathrm{H}_{\| \mathrm{i}}=\mathrm{S}_{\mathrm{y}} \frac{\mathrm{E}_{0}}{\eta_{1}} \mathrm{e}^{-\mathrm{j} \beta_{1}\left(x \sin \theta_{\mathrm{i}}+\mathrm{z} \cos \theta_{\mathrm{i}}\right)}
$$

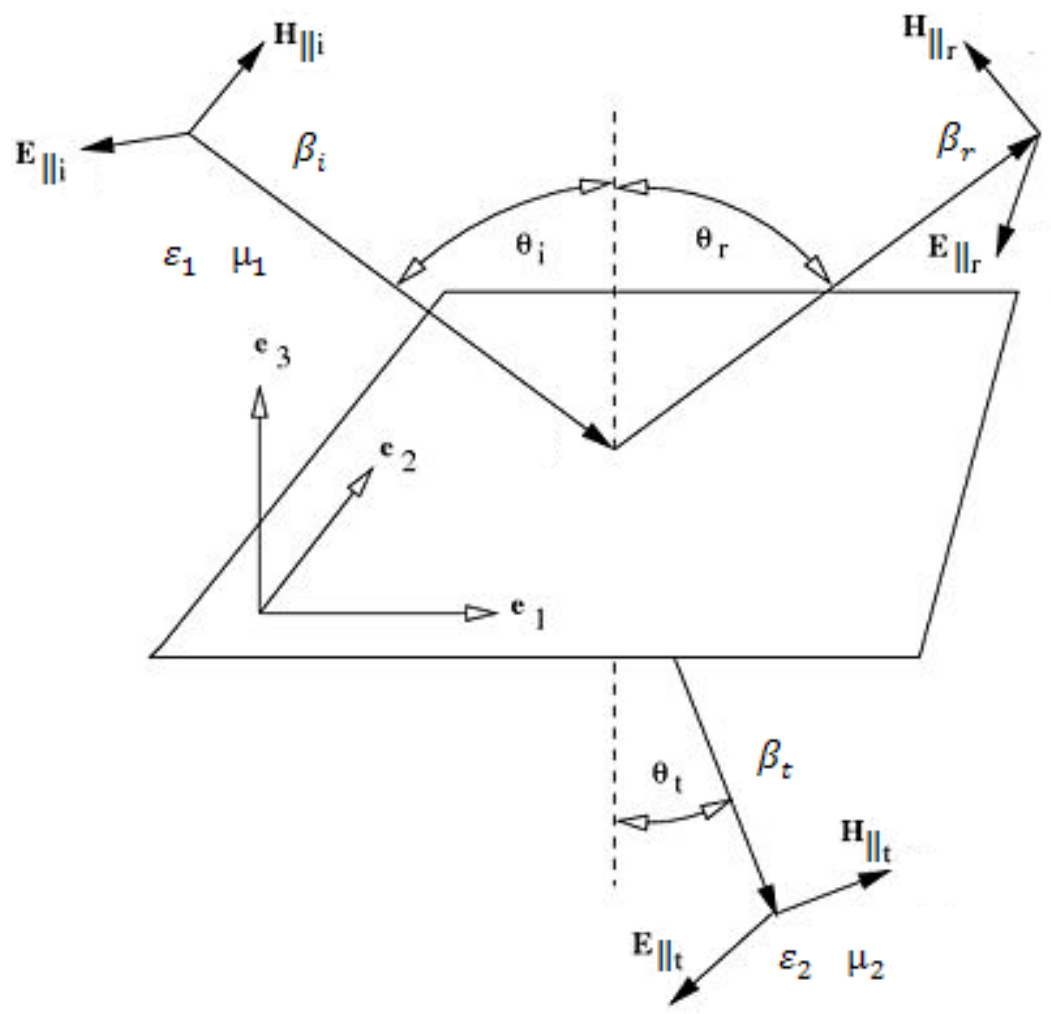

Fig. 2.9 Plane Wave reflection and transmission for parallel polarization

$$
E_{\| r}=\left(S_{z} \sin \theta_{r}+S_{x} \cos \theta_{r}\right) \Gamma_{\|}^{b} E_{0} e^{-j \beta_{1}\left(x \sin \theta_{r}-z \cos \theta_{r}\right)}
$$

$$
\begin{gathered}
\mathrm{H}_{\| \mathrm{r}}=-\mathrm{S}_{\mathrm{y}} \frac{\Gamma_{\|}^{\mathrm{b}} \mathrm{E}_{0}}{\eta_{1}} \mathrm{e}^{-\mathrm{j} \beta_{1}\left(x \sin \theta_{\mathrm{r}}-\mathrm{z} \cos \theta_{\mathrm{r}}\right)} \\
\mathrm{E}_{\| \mathrm{t}}=\left(-\mathrm{S}_{\mathrm{z}} \sin \theta_{\mathrm{t}}+\mathrm{S}_{\mathrm{x}} \cos \theta_{\mathrm{t}}\right) \mathrm{T}_{\|}{ }^{\mathrm{b}} \mathrm{E}_{0} \mathrm{e}^{-\mathrm{j} \beta_{2}\left(\mathrm{x} \sin \theta_{\mathrm{t}}+\mathrm{z} \cos \theta_{\mathrm{t}}\right)} \\
\mathrm{H}_{\| \mathrm{t}}=\mathrm{S}_{\mathrm{y}} \frac{\mathrm{T}_{\|}^{\mathrm{b}} \mathrm{E}_{0}}{\eta_{2}} \mathrm{e}^{-\mathrm{j} \beta_{2}\left(\mathrm{x} \sin \theta_{\mathrm{t}}+\mathrm{z} \cos \theta_{\mathrm{t}}\right)}
\end{gathered}
$$


By using Snell's Law the equations for the reflection and transmission coefficient can be written as

$$
\begin{gathered}
\Gamma^{\mathrm{b}}=\frac{\eta_{2} \cos \theta_{\mathrm{t}}-\eta_{1} \cos \theta_{\mathrm{i}}}{\eta_{2} \cos \theta_{\mathrm{t}}+\eta_{1} \cos \theta_{\mathrm{i}}}=\frac{\sqrt{\frac{\mu_{2}}{\epsilon_{2}}} \cos \theta_{\mathrm{t}}-\sqrt{\frac{\mu_{1}}{\epsilon_{1}}} \cos \theta_{\mathrm{i}}}{\sqrt{\frac{\mu_{2}}{\epsilon_{2}}} \cos \theta_{\mathrm{t}}+\sqrt{\frac{\mu_{1}}{\epsilon_{1}}} \cos \theta_{\mathrm{i}}} \\
\mathrm{T}^{\mathrm{b}}=\frac{2 \eta_{2} \cos \theta_{\mathrm{i}}}{\eta_{2} \cos \theta_{\mathrm{t}}+\eta_{1} \cos \theta_{\mathrm{i}}}=1+\Gamma^{\mathrm{b}}=\frac{2 \sqrt{\frac{\mu_{2}}{\epsilon_{2}}} \cos \theta_{\mathrm{i}}}{\sqrt{\frac{\mu_{2}}{\epsilon_{2}}} \cos \theta_{\mathrm{t}}+\sqrt{\frac{\mu_{1}}{\epsilon_{1}}} \cos \theta_{\mathrm{i}}}
\end{gathered}
$$

\subsubsection{Brewster's Angle}

Brewster's angle [14] $\theta_{\mathrm{B}}$ is defined as the incidence angle $\theta_{\mathrm{i}}$, at which the Fresnel reflection coefficient $\Gamma^{\mathrm{b}}=0$.

\subsection{Perpendicular Polarization}

For Perpendicular Polarization, the Brewster's angle $\theta_{\mathrm{B}_{\perp}}$ can be written as

$$
\sin \theta_{\mathrm{B}_{\perp}}=\sqrt{\frac{1-\left(\mu_{1} \varepsilon_{2} / \mu_{2} \varepsilon_{1}\right)}{1-\left(\varepsilon_{1} / \varepsilon_{2}\right)^{2}}}
$$

$\theta_{\mathrm{B}_{\perp}}$ does not exist for nonmagnetic materials, that is when $\mu_{1}=\mu_{2}$.

\subsection{Parallel Polarization}

For parallel polarization, Brewster's angle $\theta_{\mathrm{B} \|}$ can be written as

$$
\sin \theta_{B} \|=\sqrt{\frac{1-\left(\mu_{2} \varepsilon_{1} / \varepsilon_{2} \mu_{1}\right)}{1-\left(\varepsilon_{1} / \varepsilon_{2}\right)^{2}}}
$$

For nonmagnetic materials, Brewster's angle $\theta_{\mathrm{B}} \|$ can be written as

$$
\theta_{\mathrm{B} \|}=\sin ^{-1} \sqrt{\frac{1}{1+\left(\varepsilon_{1} / \varepsilon_{2}\right)}}
$$




$$
\text { For } \mu_{1}=\mu_{2}, \theta_{\mathrm{B} \|}=\tan ^{-1} \sqrt{\frac{\varepsilon_{2}}{\varepsilon_{1}}}
$$

\subsection{Permittivity of Concrete}

The permittivity of a concrete slab [7] has been modeled assuming that it is a lossy dielectric, and that a slab has a real part and an imaginary part. The permittivity of a concrete slab can be written as

$$
\widehat{\varepsilon}=\varepsilon^{\prime}-\varepsilon^{\prime \prime}
$$

$\varepsilon^{\prime}$ is the real part of complex permittivity of a concrete slab and $\varepsilon^{\prime \prime}$ is the imaginary part of permittivity of a concrete slab. By modeling a concrete slab as a Debye material [7], its frequency dependent complex relative permittivity obeys the following

$$
\widehat{\varepsilon}(\omega)=\varepsilon^{\prime}(\omega)-\varepsilon^{\prime \prime}(\omega)
$$

Table .1 Fitted Parameters for the Concrete Samples [7]

\begin{tabular}{|c|c|c|c|c|}
\hline $\begin{array}{c}\text { Moisture } \\
\text { Content }\end{array}$ & $\varepsilon_{\text {static }}$ & $\varepsilon_{\infty}$ & $\tau$ & $\sigma_{\mathrm{dc}}$ \\
\hline $0.2 \%$ & $4.8 \pm 0.002$ & $4.507 \pm 0.002$ & $0.82 \pm 0.01$ & $6.06 \times 10^{-4} \pm$ \\
$12 \%$ & $12.84 \pm 0.03$ & $7.42 \pm 0.02$ & $0.611 \pm 0.006$ & $0.06 \times 10^{-4}$ \\
& & & & $20.6 \times 10^{-4} \pm$ \\
& & & & $0.2 \times 10^{-4}$ \\
\hline
\end{tabular}

$$
\widehat{\varepsilon}(\omega)=\varepsilon_{\infty}-\frac{\Delta \varepsilon}{\left(1+\omega^{2} \tau^{2}\right)}-j \frac{\Delta \varepsilon \omega \tau}{\left(1+\omega^{2} \tau^{2}\right)}
$$

Where $\Delta \varepsilon=\varepsilon_{\text {static }}-\varepsilon_{\infty}$ is the difference between the values of the real part of the complex relative permittivity, and $\tau$ is the relaxation time. The above equation represents the first model of relative permittivity of a concrete slab. In order to take into account the additional energy 
loss due to conductivity [7], an additional term is added to the imaginary part in the above equation to form the second model of relative permittivity of a concrete slab.

$$
\varepsilon_{\mathrm{r}, \mathrm{eff}}^{\prime \prime}(\omega)=\frac{\Delta \varepsilon \omega \tau}{\left(1+\omega^{2} \tau^{2}\right)}+\frac{\sigma_{\mathrm{dc}}}{\omega \varepsilon_{0}}=\frac{\sigma_{\mathrm{eff}}(\omega)}{\omega \varepsilon_{0}}
$$

$$
\widehat{\varepsilon}(\omega)=\varepsilon^{\prime}(\omega)-\mathrm{j} \varepsilon^{\prime \prime}, \mathrm{eff}(\omega)
$$

$\sigma_{\mathrm{dc}}$ is the dc conductivity of a concrete slab, $\varepsilon_{0}$ is the permittivity of free space and $\sigma_{\text {eff }}$ is the effective conductivity of a concrete slab.
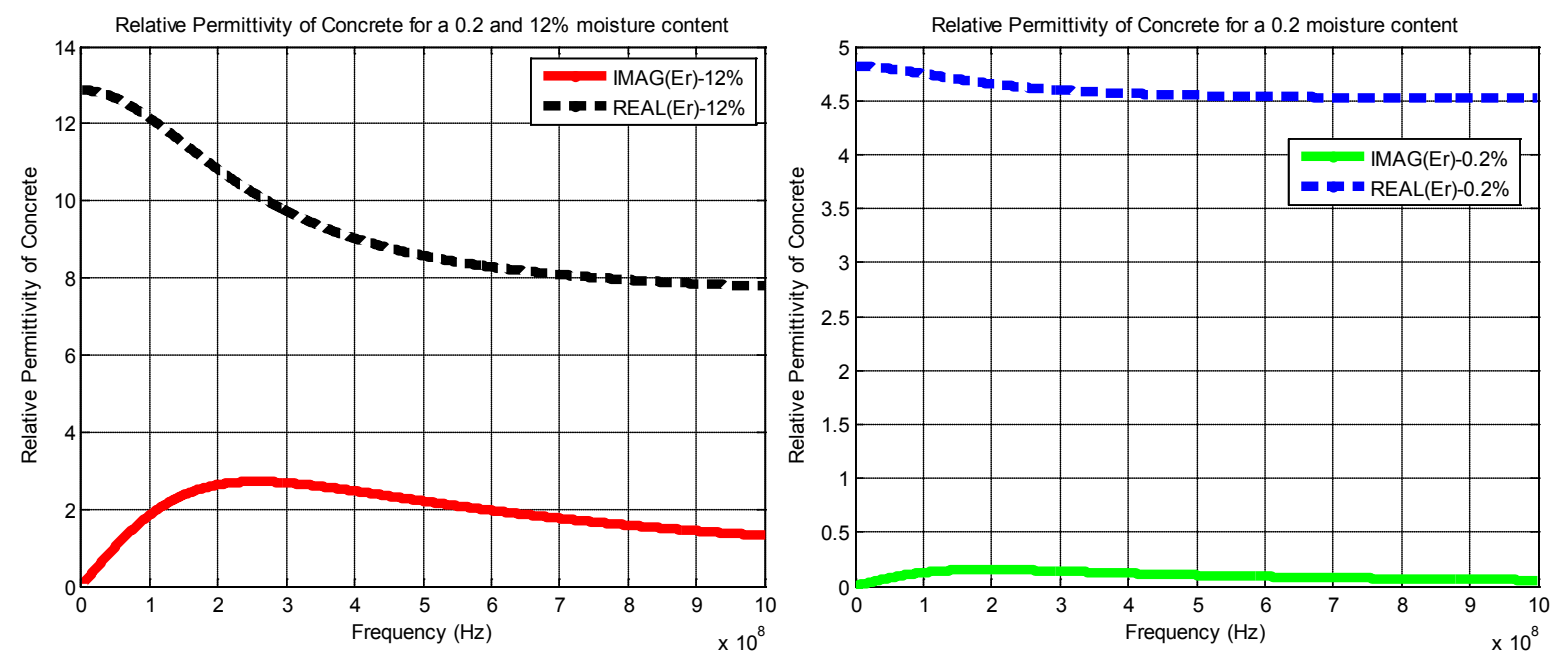

Fig. 2.10 Relative Permittivity of Concrete for the first Debye Model

The relative permittivity of a concrete slab vs. frequency $(\mathrm{Hz})$ was plotted. In the above graphs, the imaginary part and the real part of relative permittivity vary with the frequency. The real part of relative permittivity of a concrete slab is high at low frequencies, and slowly decreases as the frequency increases and reaches a stable point. The imaginary part of the relative permittivity of a concrete slab reaches a peak value, and then slowly decreases as the 
frequency increases. The imaginary part of the relative permittivity of a concrete slab was plotted for the second Debye model.

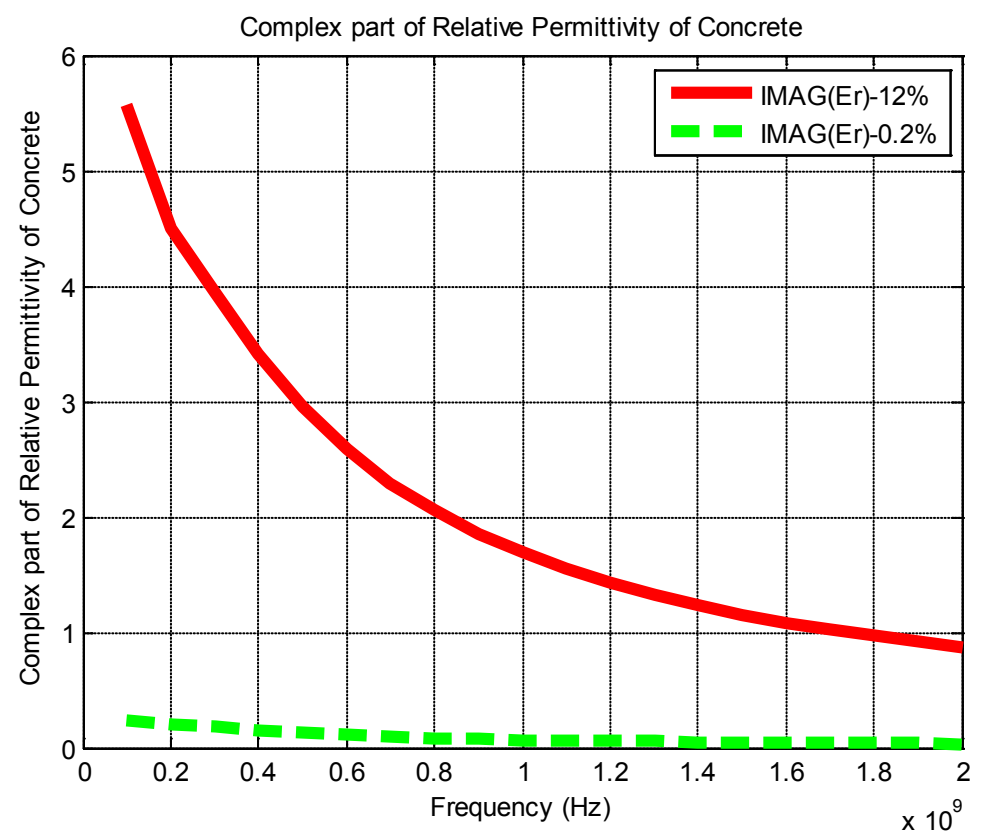

Fig. 2.11 Relative Permittivity of Concrete for the second Debye Model

The imaginary part of relative permittivity decreases as the frequency increases for both the moisture content values of $12 \%$ and $0.2 \%$.

\subsection{Radio Frequency Spectrum}

Radio communications [16] involve the usage of electromagnetic waves of different frequency spectrums. Different frequency ranges are used for different applications. Since the research presented in this dissertation focuses on reconfigurable antenna design, it is important to understand different frequency ranges, and the services that use these frequency ranges in radio wave communication.

\subsubsection{Extremely Low and Very Low Frequencies (ELF \& VLF) $(<30 \mathrm{KHz})$}

Communications through sea water, where transmitting a signal is difficult because of bandwidth limitations, use frequencies below $3 \mathrm{KHz}$. Propagation in the ELF and the VLF range is by surface wave, and by the earth-ionosphere waveguide [16]. The effective height of 
the ionosphere in the ELF band is approximately $90 \mathrm{Km}$. Submarines that communicate with land bases through ocean water use the ELF band. Huge land based transmitting antennas are required to transmit the signal, and relatively low data rates are possible. Sea water [16] is highly conductive, and the attenuation in one skin depth of sea water or any other dielectric is $8.86 \mathrm{~dB}$. Since the attenuation in sea water is very high, the longest wavelengths have to be used for the attenuation to be kept to usable values. The propagation constant $\mathrm{k}_{\mathrm{w}}$ in sea water is complex, has high conductivity losses, and is given by

$$
\mathrm{k}_{\mathrm{w}}=\mathrm{k} \sqrt{\varepsilon_{\mathrm{w}}-\mathrm{j} \frac{\sigma_{\mathrm{w}}}{\omega \varepsilon_{0}}}
$$

\subsubsection{Ionosphere}

The ionosphere is the upper region of the atmosphere [16], approximately $50 \mathrm{Km}$ above the surface of the earth, where atmospheric gasses have been ionized by solar flux and cosmic radiation. Ionization occurs because of rare atmospheric gases and radiation caused by atmospheric attenuation that exists at the highest and the lowest altitudes of the ionosphere respectively. Up to $80 \mathrm{Km}$ altitude [16], the earth's dry atmosphere is well mixed. Dissociation of ions varies with altitude above $80 \mathrm{~km}$ of the earth's surface because of varying densities of ionized gases.

\subsubsection{Low and Medium Frequencies ( $\mathrm{LF} \& \mathrm{MF})(30 \mathrm{KHz}$ to $3 \mathrm{MHz})$}

Marine and aeronautical radio navigations [16] use the LF band from $30 \mathrm{KHz}$ to 500 KHz. Atmospheric noise is a major factor in the LF and MF frequency bands. Amplitude modulation (AM) uses the band segment between $535 \mathrm{KHz}$ and $1705 \mathrm{KHz}$. Ground wave propagation [16] utilizes the MF band from $300 \mathrm{KHz}$ to $3 \mathrm{MHz}$. Ionospheric absorption is high during the daytime in the LF and the MF bands. 


\subsubsection{High Frequencies (HF) (3 to $30 \mathrm{MHz})$}

Worldwide radio communications [16] that are designed on the basis of ionospheric reflections use the HF band. Ionospheric transmission provides a channel of small attenuation. Narrow band applications [16] of bandwidth less than $3 \mathrm{KHz}$ use the HF band.

\subsubsection{Very High Frequencies and Ultrahigh Frequencies (VHF \& UHF) (30 MHz to $3 \mathrm{GHz})$}

Mobile communications services (MCS), personal communication services (PCS), and satellite-based services utilize the VHF and the UHF frequency ranges. Geomagnetic activity [16] causes significant ionospheric reflections from 50 to $60 \mathrm{MHz}$. Cellular telephone services [16] use the frequency range between 800 and $900 \mathrm{MHz}$. Paging and messaging use the 900 $\mathrm{MHz}$ band, and the PCS uses the bands from 1700 to $2200 \mathrm{MHz}$. Personal and local area networks use frequencies above $2400 \mathrm{MHz}$.

\subsubsection{Above Ultrahigh Frequencies (Above $3 \mathrm{GHz}$ )}

Satellite-based communications [16] use the UHF bands. Propagation is generally Line of Sight with occasional tropospheric scattering. Propagation in satellite systems [16] is done through the ionosphere, and signal polarization is rotated because of the combined effect of the earth's magnetic field and the free ion concentration.

\subsubsection{UWB Systems}

FCC regulations in the United States permit [16] Ultra Wideband (UWB) communications in the 3.1 to $10.6 \mathrm{GHz}$ frequency spectrum.

\subsection{Wireless Powering}

During the transmission of radio frequency energy from one antenna to another, the electric field is either incident parallel to the receiving antenna, or is incident at an angle. If the electric field is parallel to the receiving antenna, it receives maximum voltage supply, and if the electric field [16] is incident at an angle, it receives voltage as a function of the angle at which the electric field is incident on it. 
Table . 2 Radio Frequency Spectrum [16]

\begin{tabular}{|c|c|c|c|}
\hline Frequency & Band & Characteristics & Services \\
\hline $3 \mathrm{~Hz}-30 \mathrm{KHz}$ & ELF, VLF & $\begin{array}{l}\text { High atmospheric } \\
\text { noise, inefficient } \\
\text { antennas. }\end{array}$ & $\begin{array}{l}\text { Submarine, navigation, } \\
\text { sonar. }\end{array}$ \\
\hline $30-300 \mathrm{KHz}$ & $\mathrm{LF}$ & $\begin{array}{l}\text { High atmospheric } \\
\text { noise }\end{array}$ & $\begin{array}{l}\text { Long-range navigation } \\
\text { beacons. }\end{array}$ \\
\hline $0.3-3 \mathrm{MHz}$ & $\mathrm{MF}$ & $\begin{array}{l}\text { High atmospheric } \\
\text { noise, good ground } \\
\text { wave propagation. }\end{array}$ & $\begin{array}{l}\text { Navigation, maritime } \\
\text { communication, AM } \\
\text { broadcasting. }\end{array}$ \\
\hline 3-30 MHz & $\mathrm{HF}$ & $\begin{array}{l}\text { Moderate } \\
\text { atmospheric } \\
\text { noise, ionospheric } \\
\text { reflections that } \\
\text { provide long distance } \\
\text { links, affected by } \\
\text { solar flux }\end{array}$ & $\begin{array}{l}\text { International Shortwave } \\
\text { broadcasting } \\
\text {, ship-to-shore, } \mathrm{t} \\
\text { Telephone, telegraphy } \\
\text {, long range aircraft } \\
\text { communication, } \\
\text { amateur radio. }\end{array}$ \\
\hline $30-300 \mathrm{MHz}$ & VHF & $\begin{array}{l}\text { Ionospheric } \\
\text { reflections, line of } \\
\text { sight propagation }\end{array}$ & $\begin{array}{l}\text { Mobile, FM } \\
\text { broadcasting, air traffic } \\
\text { control, television, radio } \\
\text { navigation aids. }\end{array}$ \\
\hline $0.3-3 \mathrm{GHz}$ & UHF & $\begin{array}{l}\text { Line of sight } \\
\text { propagations, } \\
\text { efficient } \\
\text { portable antennas. }\end{array}$ & $\begin{array}{l}\text { Television, radar, } \\
\text { Global Positioning } \\
\text { Systems (GPS), PCS, } \\
\text { mobile phones, } \\
\text { wireless local area } \\
\text { networking, } \\
\text { land-mobile } \\
\text { communications, } \\
\text { satellite } \\
\text { communications }\end{array}$ \\
\hline $3-30 \mathrm{GHz}$ & SHF & $\begin{array}{l}\text { Line of sight } \\
\text { propagation }\end{array}$ & $\begin{array}{l}\text { UWB, fixed broadband, } \\
3 \mathrm{G} \text { PCS, } \\
\text { Microwave links, land- } \\
\text { mobile communication, } \\
\text { wireless LANs and } \\
\text { and PANs, fixed } \\
\text { broadband, 3G PCS. }\end{array}$ \\
\hline $30-300 \mathrm{GHz}$ & EHF & $\begin{array}{l}\text { Line of sight } \\
\text { propagation, } \\
\text { atmospheric } \\
\text { absorption }\end{array}$ & $\begin{array}{l}\text { Radar, military and } \\
\text { secure,Communications, } \\
\text { satellite links, mm-wave } \\
\text { personal-area } \\
\text { networking. }\end{array}$ \\
\hline $300-10^{7} \mathrm{GHz}$ & IR-optics & $\begin{array}{l}\text { Line of sight } \\
\text { propagation, } \\
\text { atmospheric } \\
\text { absorption }\end{array}$ & $\begin{array}{l}\text { Optical } \\
\text { communications, fiber } \\
\text { optical links. }\end{array}$ \\
\hline
\end{tabular}



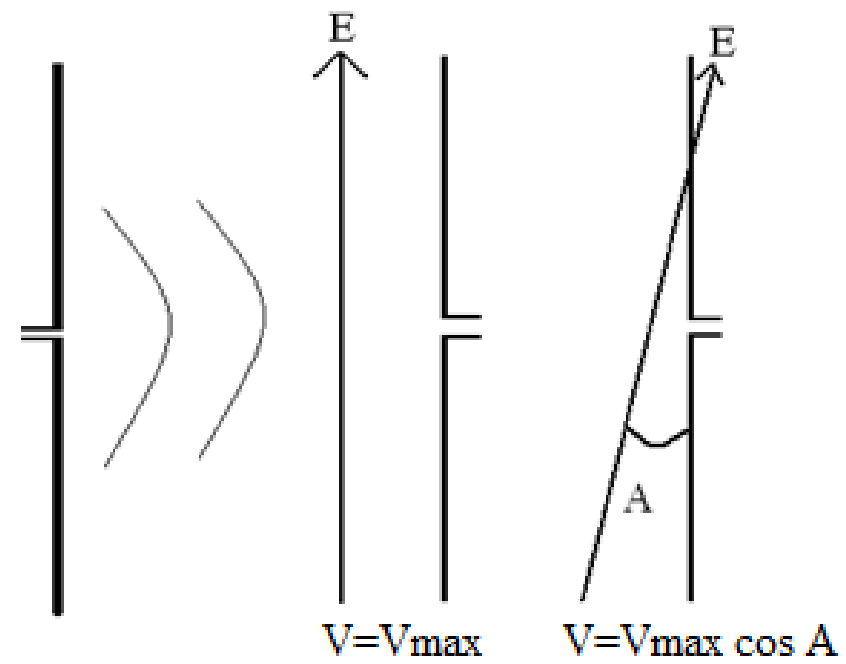

Fig. 2.12 Wireless Power Transmission

\subsubsection{Current Consumption of Typical Sensors}

A temperature sensor [9] consumes $300 \mu \mathrm{A}\left(\mu=\mathrm{Micro}=10^{-6}\right)$ for $50 \mu \mathrm{Sec}$ for a stable reading every five seconds, and a humidity sensor consumes $2.8 \mathrm{~mA}$ for $150 \mathrm{msec}$ for a stable reading every thirty seconds. The radio frequency energy incident on the receiving antenna has to be converted to electrical energy [8] to enable wireless powering.

\subsection{Antenna Simulation Parameters}

In order to understand the performance of an antenna, a detailed understanding of the simulation parameters of an antenna is necessary.

\subsubsection{S-Parameters}

When an antenna is excited at one end, the measurements of reflected current or voltage reveals the frequencies at which the antenna works best. This measurement can be done using the S-parameters. The S-parameter matrix for the 2-port network generates higher order matrices for larger networks $[56,57]$. In this case, the relationship between the reflected and the incident power waves, as well as the S-parameter matrix is given by

$$
\left(\begin{array}{l}
x_{1} \\
x_{2}
\end{array}\right)=\left(\begin{array}{ll}
S_{11} & S_{12} \\
S_{21} & S_{22}
\end{array}\right)\left(\begin{array}{l}
y_{1} \\
y_{2}
\end{array}\right)
$$




$$
\begin{aligned}
& x_{1}=S_{11} y_{1}+S_{12} y_{2} \\
& x_{2}=S_{21} y_{1}+S_{22} y_{2}
\end{aligned}
$$

If port 2 is terminated in a load identical to the system impedance then, by the maximum power transfer theorem, $\mathrm{x}_{2}$ will be totally absorbed making $\mathrm{y}_{2}$ equal to zero, and vice versa. Thus, the above two equations are reduced to

$$
\begin{aligned}
& S_{11}=\frac{x_{1}}{y_{1}} \text { and } S_{21}=\frac{x_{2}}{y_{1}} \\
& S_{12}=\frac{x_{1}}{y_{2}} \text { and } S_{22}=\frac{x_{2}}{y_{2}}
\end{aligned}
$$

Each parameter can be defined as:

- $\mathrm{S}_{11}$ is the input port voltage reflection coefficient

- $\mathrm{S}_{12}$ is the reverse voltage gain

- $\mathrm{S}_{21}$ is the forward voltage gain

- $\mathrm{S}_{22}$ is the output port voltage reflection coefficient

The frequency at which $S_{11}$ is least is the resonating frequency. It is the frequency at which the antenna works best.

\subsubsection{Directivity}

Directivity of an antenna [13] is defined as the ratio of radiation intensity in a given direction from the antenna, to the average radiation intensity in all other directions.

$$
\mathrm{D}=\frac{\mathrm{U}}{\mathrm{U}_{0}}=\frac{4 \pi \mathrm{U}}{\mathrm{P}_{\mathrm{rad}}}
$$


If the direction is not specified, then the direction of maximum radiation intensity is used and is expressed as

$$
\mathrm{D}_{\max }=\mathrm{D}_{0}=\frac{\left.\mathrm{U}\right|_{\max }}{\mathrm{U}_{0}}=\frac{\mathrm{U}_{\max }}{\mathrm{U}_{0}}=\frac{4 \pi \mathrm{U}_{\max }}{\mathrm{P}_{\mathrm{rad}}}
$$

$\mathrm{D}=$ directivity (no dimensions)

$\mathrm{D}_{0}=$ maximum directivity (no dimensions)

$\mathrm{U}=$ radiation intensity (W/unit solid angle)

$\mathrm{U}_{\max }=$ maximum radiation intensity (W/unit solid angle)

$\mathrm{U}_{0}=$ radiation intensity of isotropic source

$\mathrm{P}_{\mathrm{rad}}=$ total radiated power $(\mathrm{W})$

\subsubsection{VSWR}

In a transmission line, a standing wave ratio (SWR) [58] is the ratio of the amplitude of a partial standing wave at an antinode (maximum), to the amplitude at an adjacent node (minimum). Generally, the SWR is defined as a voltage ratio called the voltage standing wave ratio (VSWR). A VSWR value 1.2:1 denotes a maximum standing wave amplitude, which is 1.2 times greater than the minimum standing wave value. The VSWR for an antenna has to be lower than 2.

\subsection{Reconfigurable Antennas}

A reconfigurable antenna consists of switches and radiating parts. The Reconfigurable Sierpinski Gasket antenna (RSGA) [2, 36] shown in fig. 2.13 consists of three similar triangular radiating parts. The top triangle is connected to the bottom two triangles by switches, as shown in fig. 2.13. The switches can be turned on or off, and the size of the antenna as well as radiation patterns can be varied. The Reconfigurable Sierpinski Gasket antenna shown in 


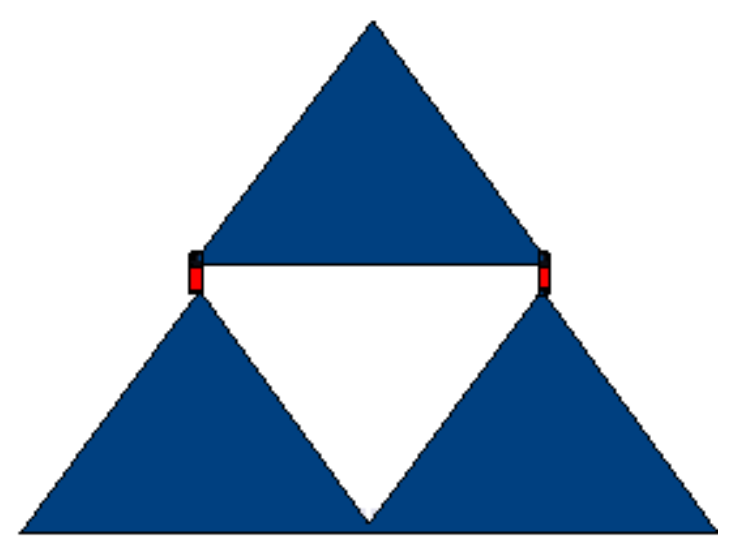

Fig. 2.13 Reconfigurable Sierpinski Gasket Antenna

fig. 2.13, consists of five feeding points (the three free vertices of the three triangles and the two switches). This antenna can be used over a range of frequencies from 1 to $20 \mathrm{GHz}$, over which it resonates at two frequencies.

\subsubsection{Reconfigurable Sierpinski Gasket Antenna}

Two important parameters that constitute into forming a Sierpinski Gasket Antenna are the base $b$ and the height $h$. To extend the concept of self similarity to antennas, the right half plane of a right angled triangle is joined to the left half plane of another right angled triangle, to form one triangle.

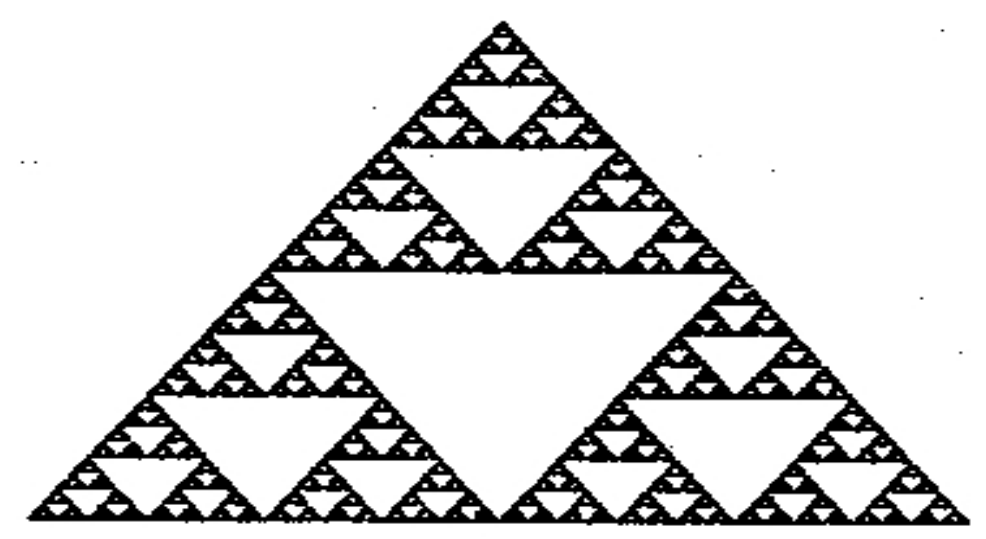

Fig. 2.14 Reconfigurable Sierpinski Gasket Antenna with iterations

If one iteration is added to the triangle already formed, then two more triangles are added to the existing geometry $[2,36]$. It is important to understand that the parameters $\mathrm{h}$ and $\mathrm{s}$ 
become half with every iteration, which is an addition of a new triangle, as is shown in fig. 2.14. The radiation pattern of an antenna is related to the distributions of currents on its surface, so if the amount of current flowing to a particular part of the antenna is varied at different frequencies, then its radiation pattern varies. Thus the characteristics of self similarity of a fractal antenna are used in the design $[2,18,38]$ as the fractal antennas radiate at different frequencies.

\subsubsection{Reworked Results of the Reconfigurable Sierpinski Gasket Antenna}

The base of the entire Sierpinski triangle is $3.74 \mathrm{~mm}$ and the height is $3.26 \mathrm{~mm}$. The antenna was excited at the top vertices by using a lumped port, as shown in fig. 2.15. The antenna was simulated in the frequency range of 1 to $20 \mathrm{GHz}$. The first iteration is when all the triangles are radiating, and the second iteration is when the top two triangles are radiating. All simulations were done using Ansoft HFSS v11. The height of the substrate was taken to be $1.5 \mathrm{~mm}$. The material of the substrate was RT Durroid 5880. Below the substrate, a copper ground plane was placed. The dimensions of the ground plane and the substrate are $8 \mathrm{~mm} \times 4 \mathrm{~mm}$. The thickness of the copper sheets was taken as $0.017 \mathrm{~mm}$.

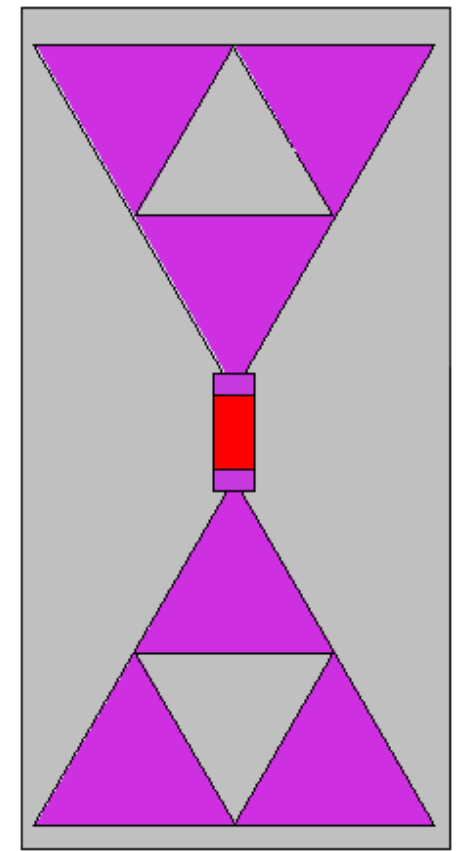

Fig. 2.15 Simulated Reconfigurable Sierpinski Gasket Antenna 


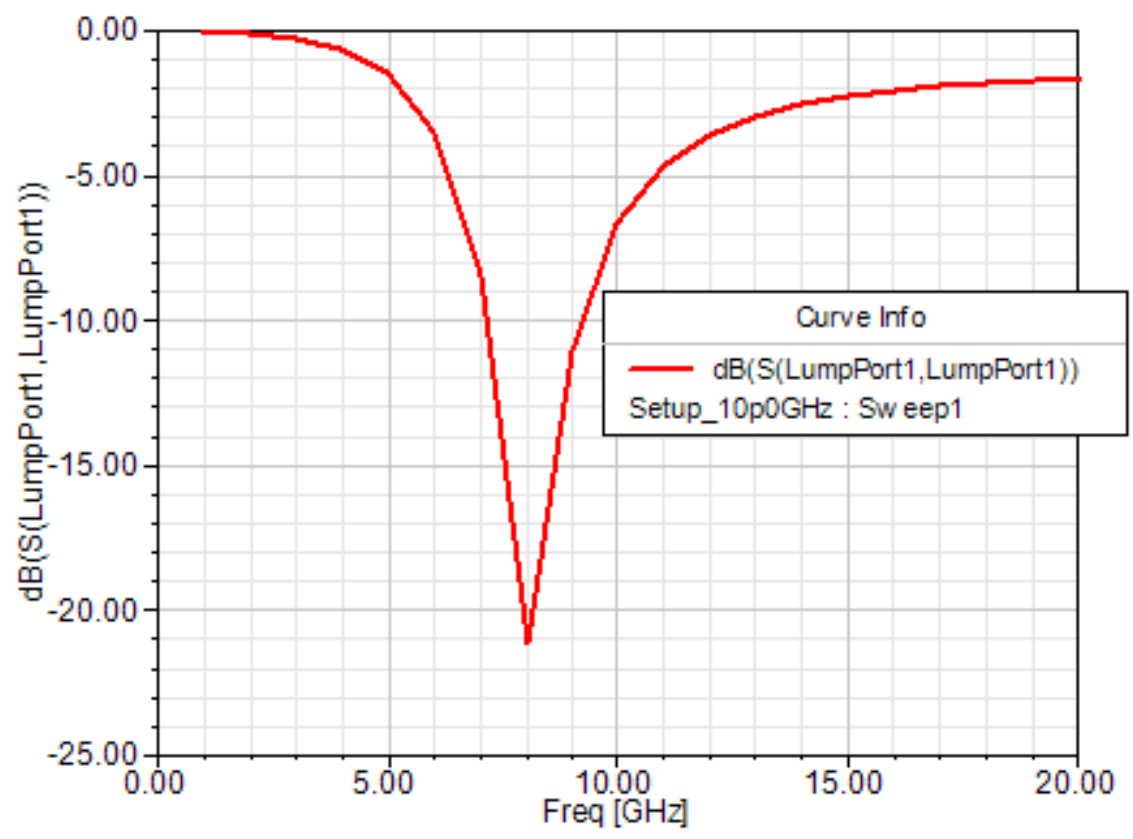

Fig. 2.16 Input Return Loss $\left(S_{11}\right)$ for the first iteration

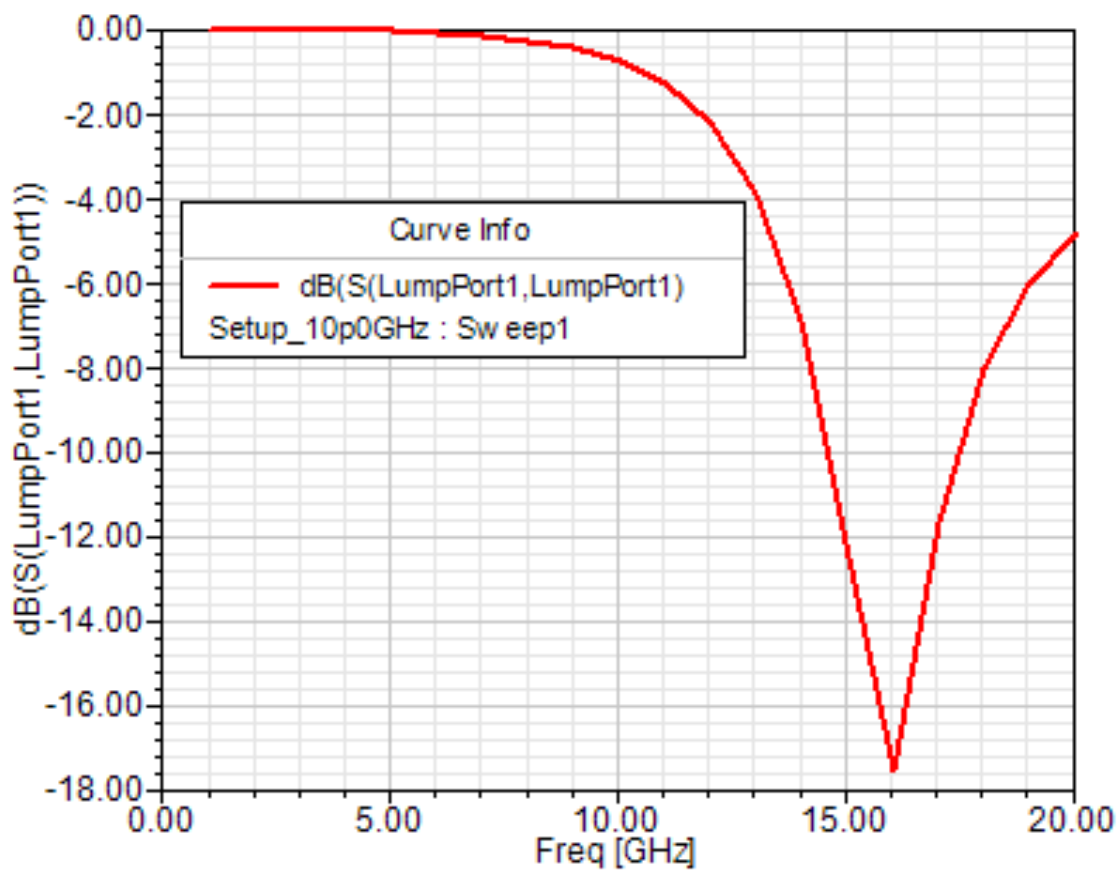

Fig. 2.17 Input Return Loss $\left(S_{11}\right)$ for the second iteration 


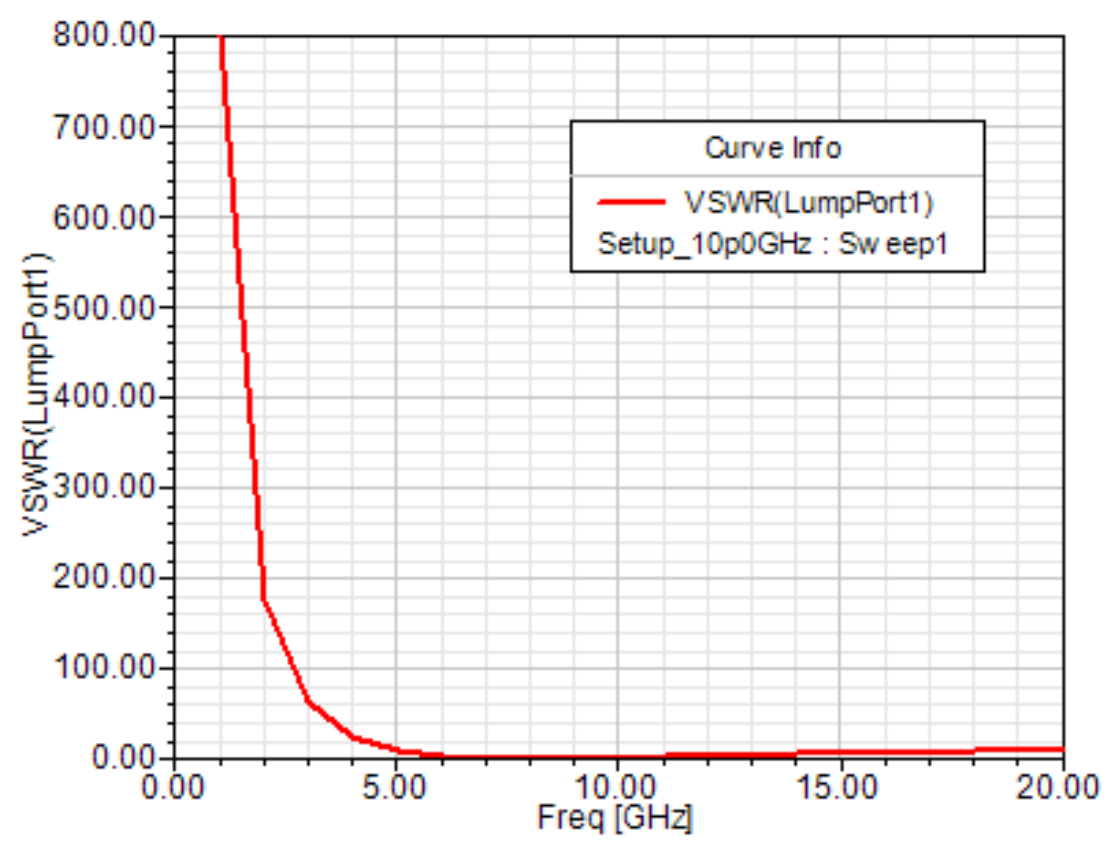

Fig. 2.18 VSWR for the first iteration

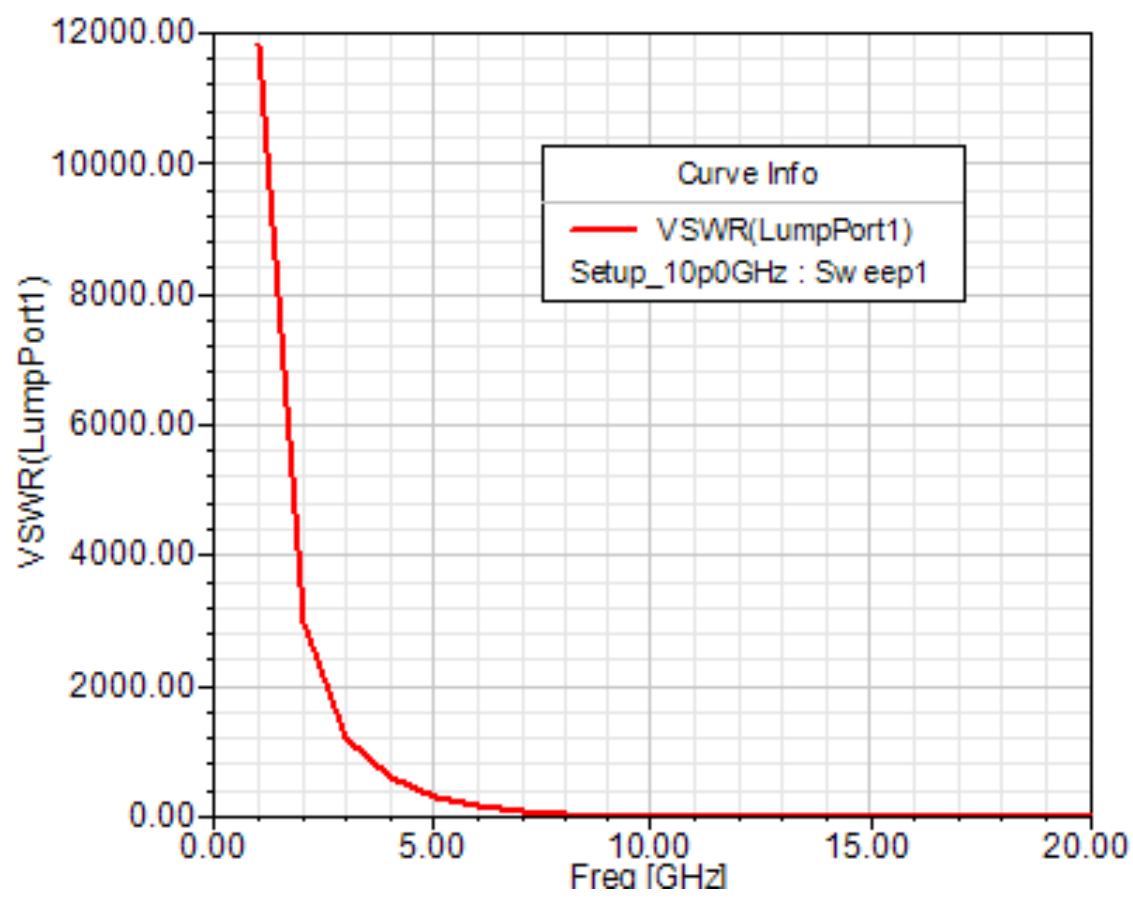

Fig. 2.19 VSWR for the second iteration 


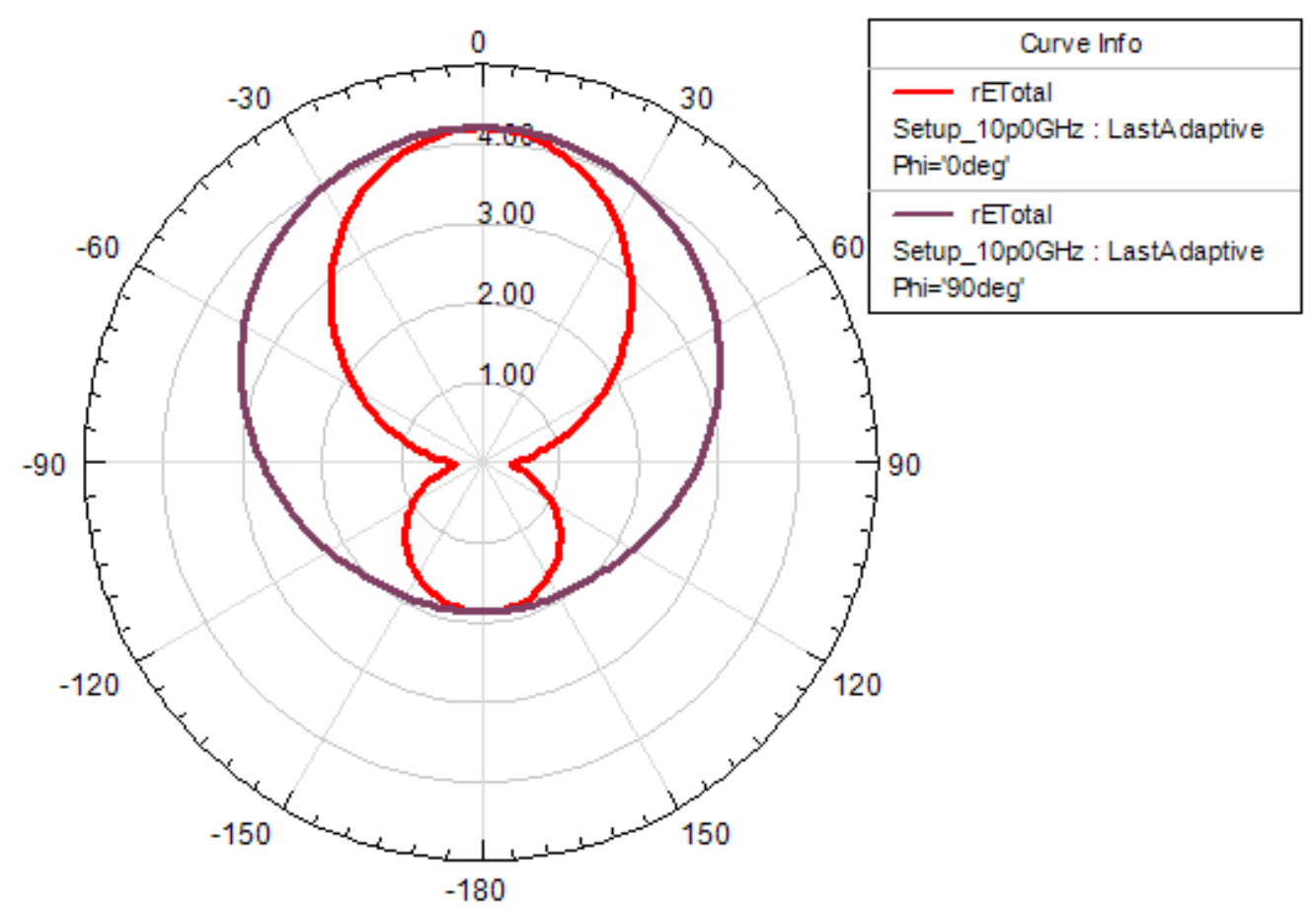

Fig. 2.20 Radiation Pattern for the first iteration

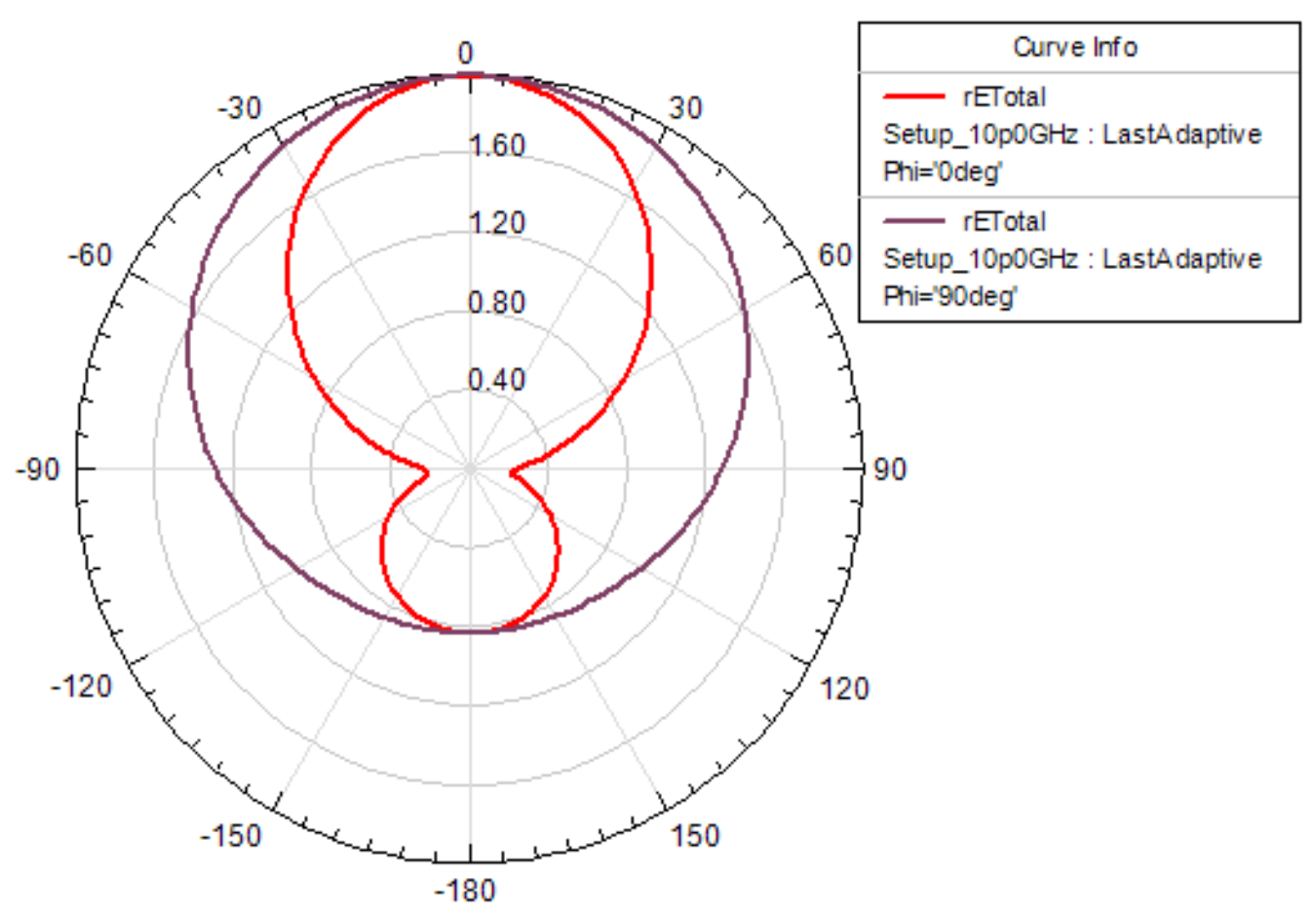

Fig. 2.21 Radiation Pattern for the second iteration 


\subsubsection{Data Analysis and Shortcomings}

Input Return $\operatorname{Loss}\left(\mathrm{S}_{11}\right)$ from fig. 2.16 for the first iteration shows that there is a resonating frequency at $8 \mathrm{GHz}$. Input Return $\operatorname{Loss}\left(\mathrm{S}_{11}\right)$ from fig. 2.17 for the second iteration shows that there is a resonating frequency at $16 \mathrm{GHz}$. VSWR for the first and the second iteration shows a very high value until $8 \mathrm{GHz}$. The radiation patterns show an omnidirectional radiation pattern for this antenna. There is no justifiable mathematical design equation which can be used by an engineer to design this antenna for a specific bandwidth. The input return loss for each iteration reveals only one resonating frequency. If more iterations are to be added, then the design becomes complex.

\subsubsection{Reconfigurable Planar Inverted Fractal Antenna (RPIFA)}

The inverted planar antenna consists of two important elements: the planar radiating element and the ground plane. It is a variant of the monopole antenna, where the radiating element has been made parallel to the ground plane. The resulting electric field that emerges after exciting the RPIFA antenna is due to the radiating electric field and its ground plane. The

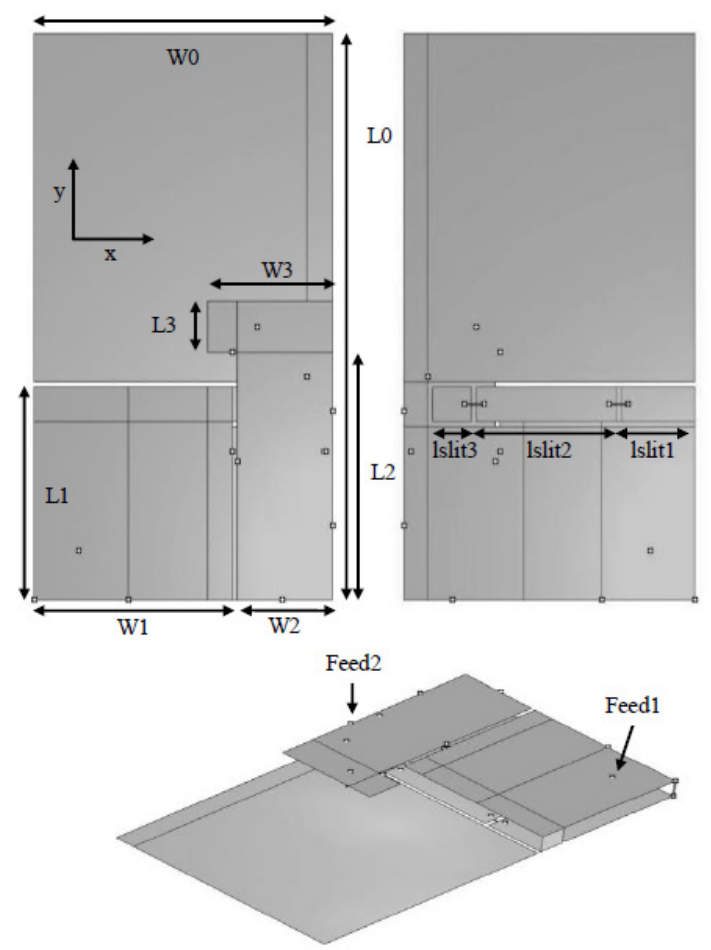

Fig. 2.22 Reconfigurable PIFA Antenna [1] 
RPIFA antenna is highly favored for its smaller size and lower profile, and is [1] widely preferred in wireless devices. PIFA antennas have many additional advantages, such as being cost effective to manufacture, very easy to fabricate, having higher bandwidth and favorable electrical performance.

\subsubsection{Reworked Results of the Reconfigurable Planar Inverted Fractal Antenna (RPIFA)}

A copper ground plane has been used for the RPIFA. The switches for this antenna [1] have been simulated as perfect electric conducting cylinders. The feeds were simulated as perfect electric conducting cylinders, which were excited by using lumped ports. The dimensions of the ground plane [1] are $\mathrm{W} 0=60 \times \mathrm{L} 0=114 \mathrm{~mm}$. At a height of $4 \mathrm{~mm}$ above the ground plane is a metallic patch of dimensions $\mathrm{L} 1=45 \times \mathrm{W} 1=40 \mathrm{~mm}$. At the end of this patch is a slit divided into three parts, each of which has a width of $4 \mathrm{~mm}$. The length of the three parts are 1 slit $1=15 \mathrm{~mm}, 1$ slit $2=15 \mathrm{~mm}$ and 1 slit $3=9 \mathrm{~mm}$ respectively. These three parts are connected by switches. The three slits were modeled as boxes of copper. The dimensions of the second $\mathrm{L}$ shaped patch element are L2 $=50 \mathrm{~mm} \times \mathrm{W} 2=19 \mathrm{~mm}$, and L3 $=10 \mathrm{~mm} \times \mathrm{W} 3=25 \mathrm{~mm}$. A total of 14 switches connect the radiating elements to the ground plane. The thickness of the copper sheets was taken as $0.017 \mathrm{~mm}$.

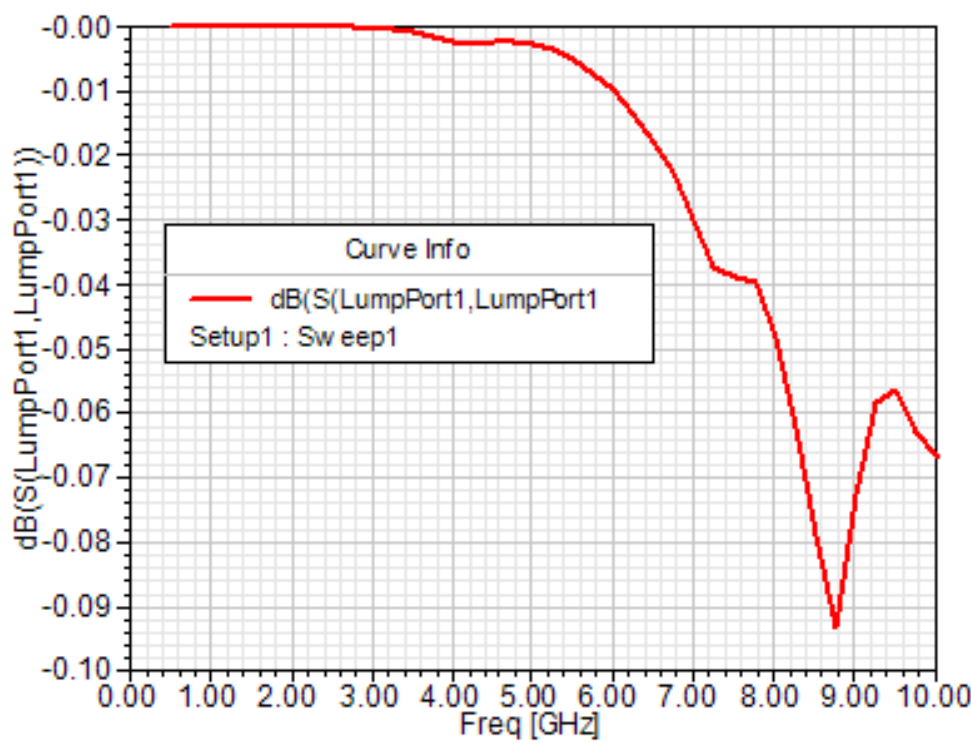

Fig. 2.23 Input Return Loss $\left(\mathrm{S}_{11}\right)$ at lumped port 1 


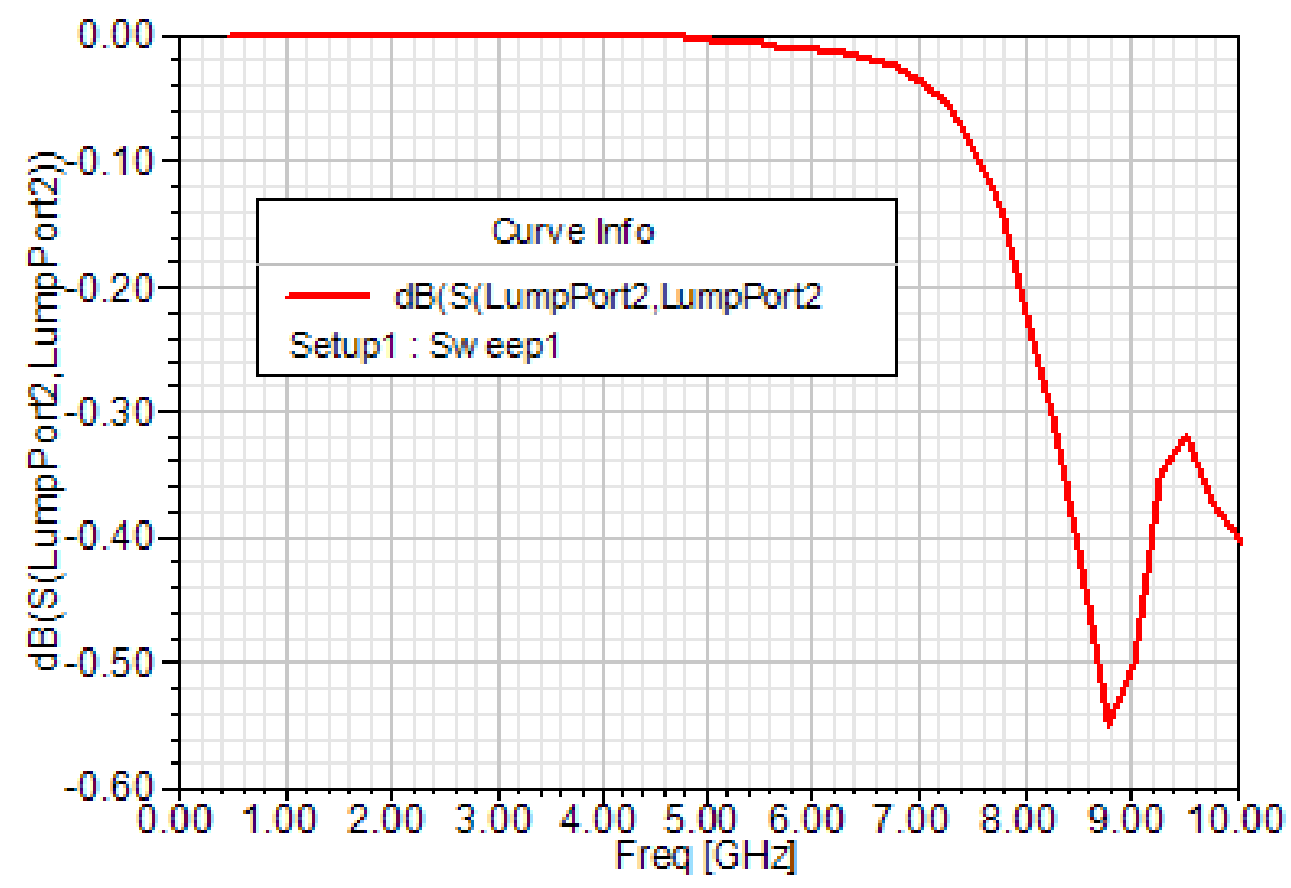

Fig. 2.24 Input Return Loss $\left(\mathrm{S}_{11}\right)$ at lumped port 2

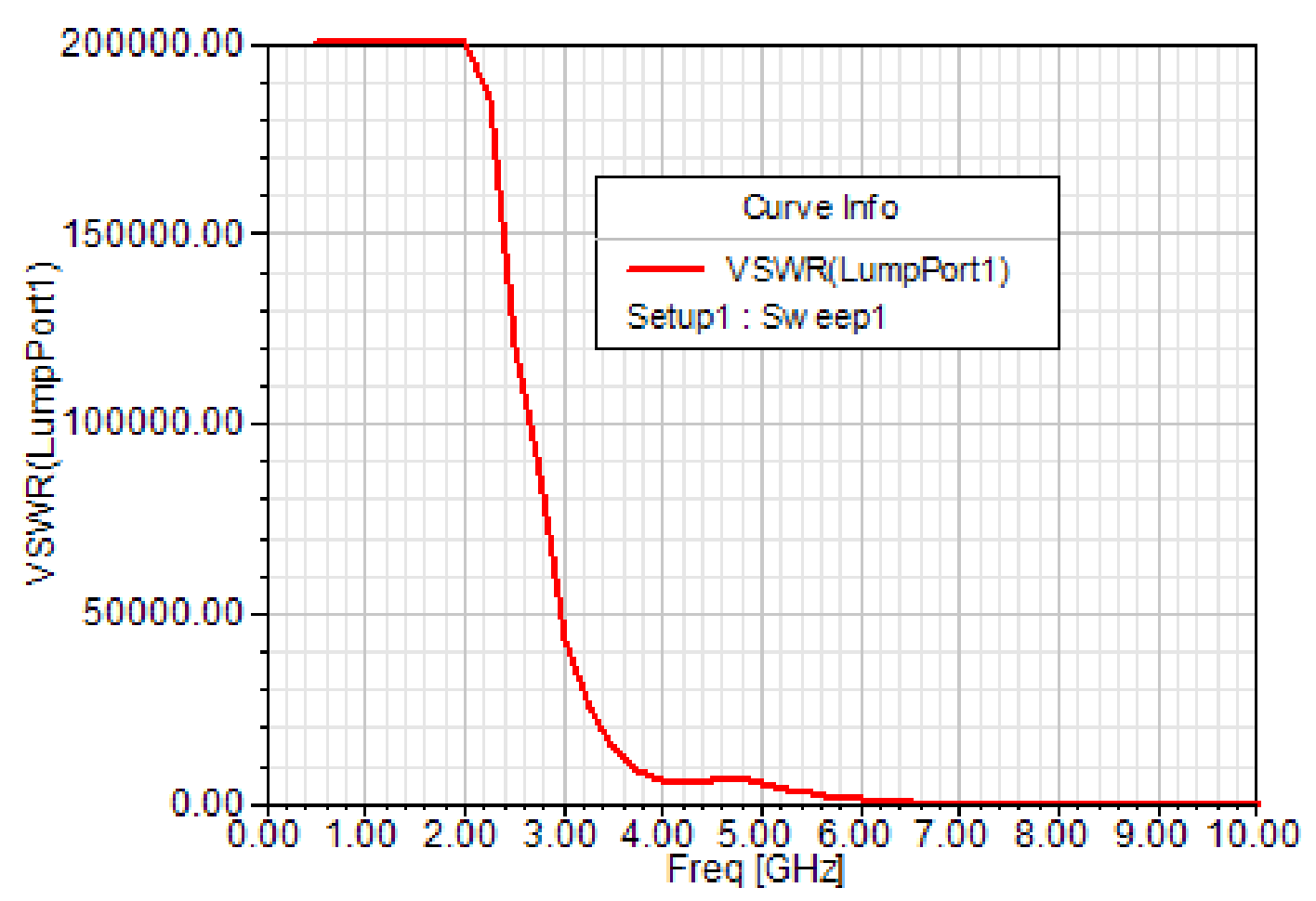

Fig. 2.25 VSWR at lumped port 1 


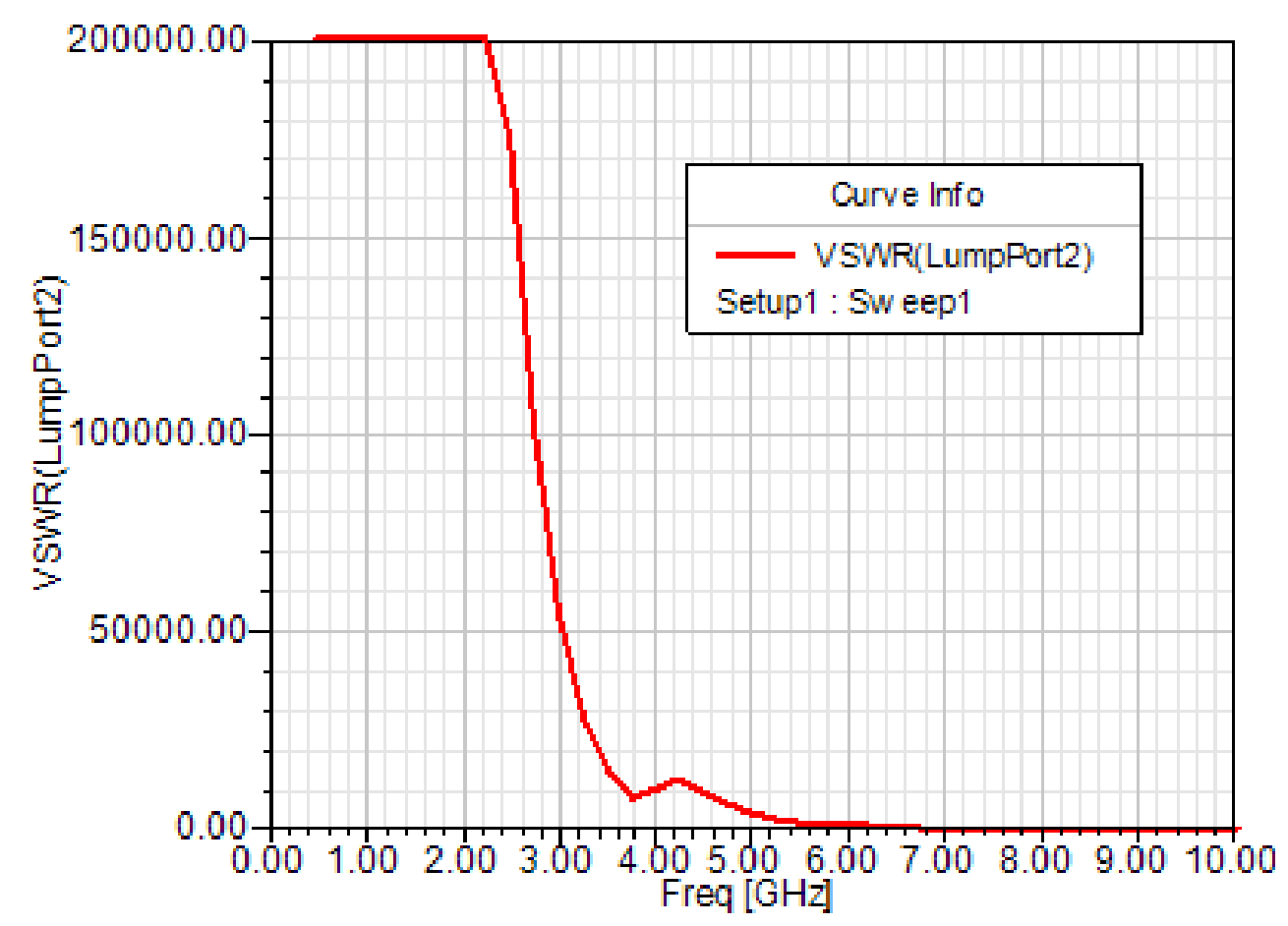

Fig. 2.26 VSWR at lumped port 2

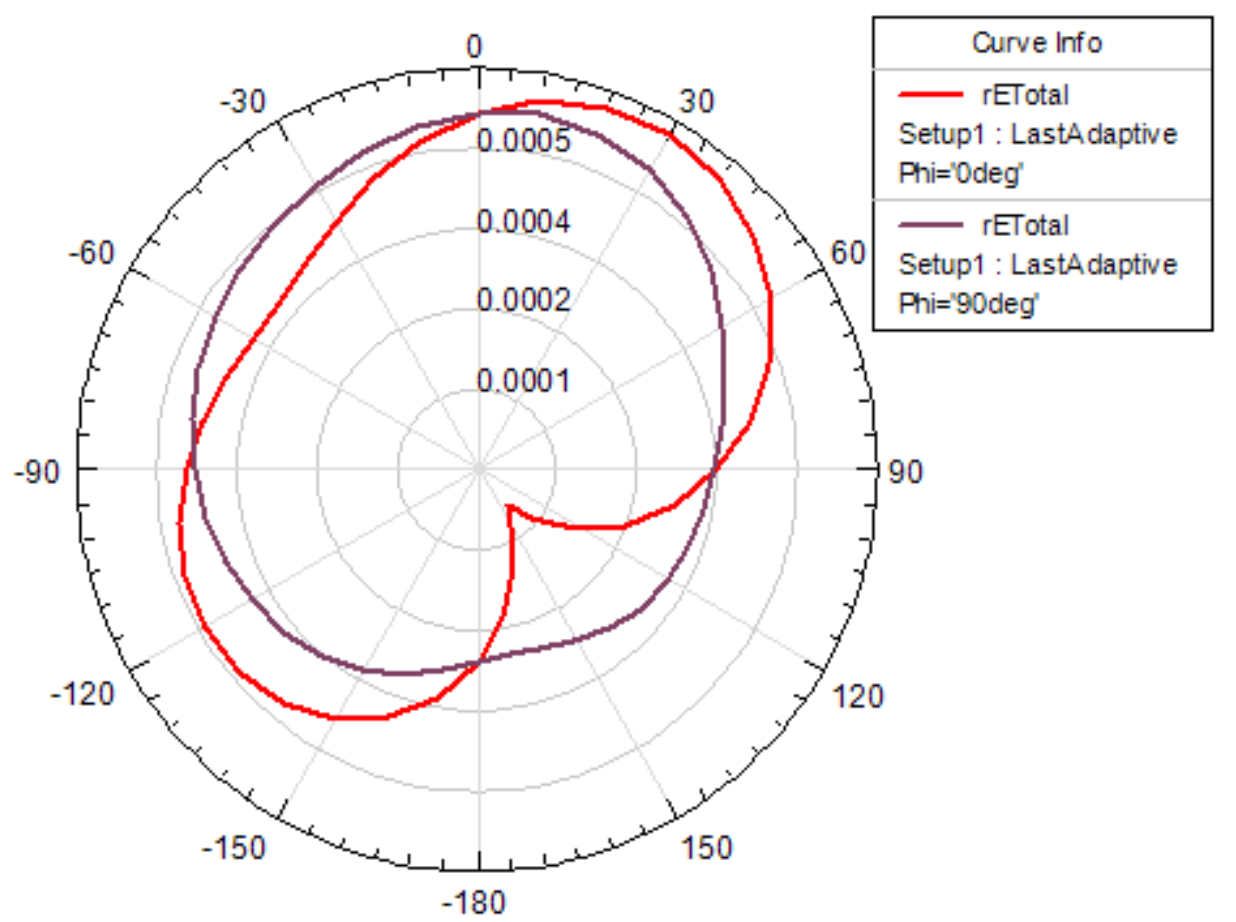

Fig. 2.27 Radiation Pattern for the Reconfigurable PIFA Antenna 


\subsubsection{Data Analysis and Shortcomings}

This antenna was simulated in the original paper [1] using a matrix along with signal processing techniques. Unfortunately, the simulation techniques used [1] were not described clearly. Though the results could change with the size in the lumped port, they are discouraging. The input return losses $\left(S_{11}\right)$ hardly show any resonating frequencies, so the antenna is hardly radiating any power. The VSWR for this antenna is also extremely high. A major setback for this antenna is that reconfigurability is not properly defined. This antenna has only one iteration with or without the presence of switches. Once again, there is no design equation to help the designer to design this antenna for a specific bandwidth.

\subsection{Summary}

The RSGA antenna works between the frequency range of $3 \mathrm{GHz}$ to $30 \mathrm{GHz}$. It can be used for UWB and fixed broadband applications. Research has to be done to check if wireless powering in a concrete slab is possible. Research has to also be done to see if reconfigurable antennas can facilitate wireless powering. Finally, a new reconfigurable antenna has to be developed which is easy to design, has mathematical design equations and has good performance. The reconfigurable antennas described in earlier sections have a lot of disadvantages, and they need to be rectified. A concrete surface needs to be recreated in Ansoft HFSS. 


\section{Chapter 3 \\ Wireless Powering in a Concrete Slab}

\subsection{Introduction}

This chapter deals with powering a sensor buried inside a concrete slab at a certain depth by delivering power to the load resistance of the sensor antenna. Antennas inside a concrete slab should be able to receive power from the electromagnetic energy that penetrates the concrete slab. If an antenna outside a concrete slab is radiating power, then that power penetrates the surface of the concrete slab and decays according to the parameters of a concrete slab. So researching the parameters of a concrete slab, as well as investigating the attenuation of the electric field as the depth increases is very important to determine the power received by an antenna. This chapter also investigates which antenna is the best to receive maximum power inside a concrete slab. As stated earlier in section 2.5, a concrete slab is a penetrable material which requires a detailed research and understanding into its electrical properties, if it has to be used in any application.

The theoretical Debye model [7] has been used to understand the behavior of a concrete slab with respect to frequency, moisture content and other factors. In the analyses, [7] a concrete slab has been treated as a dielectric material having both the real and complex part of permittivity and effective conductivity, which are dependent on frequency. Measurements have been performed to understand the frequency-dependent nature of the electrical properties of a concrete slab.

When a plane wave is incident on the surface of a concrete slab, some part of it is reflected back and the remaining passes into the slab. The power that the antenna receives depends on the polarization of the incident plane wave, as well as the attenuation due to propagation through a concrete slab. If the polarization of the incident waves and the angle of 
incidence determines the power received, then those factors become very important and have to be taken into consideration.

\subsection{Uniform Plane Waves in a Concrete Medium-Principal Axis and Oblique Angle}

Let us consider that a uniform plane wave is incident on a concrete slab. As of now, the direction and orientation of the electric field is not considered for simplicity. Since the wave is travelling from free space into a concrete slab interface, we assume that the incident electric field has a certain magnitude. This magnitude of the electric field decays with the attenuation of a concrete slab as well as the depth. Let us suppose that the incident electric field is given by

$$
\mathrm{E}_{\mathrm{i}}=\mathrm{E}
$$

The attenuation in a concrete slab was calculated using the equation

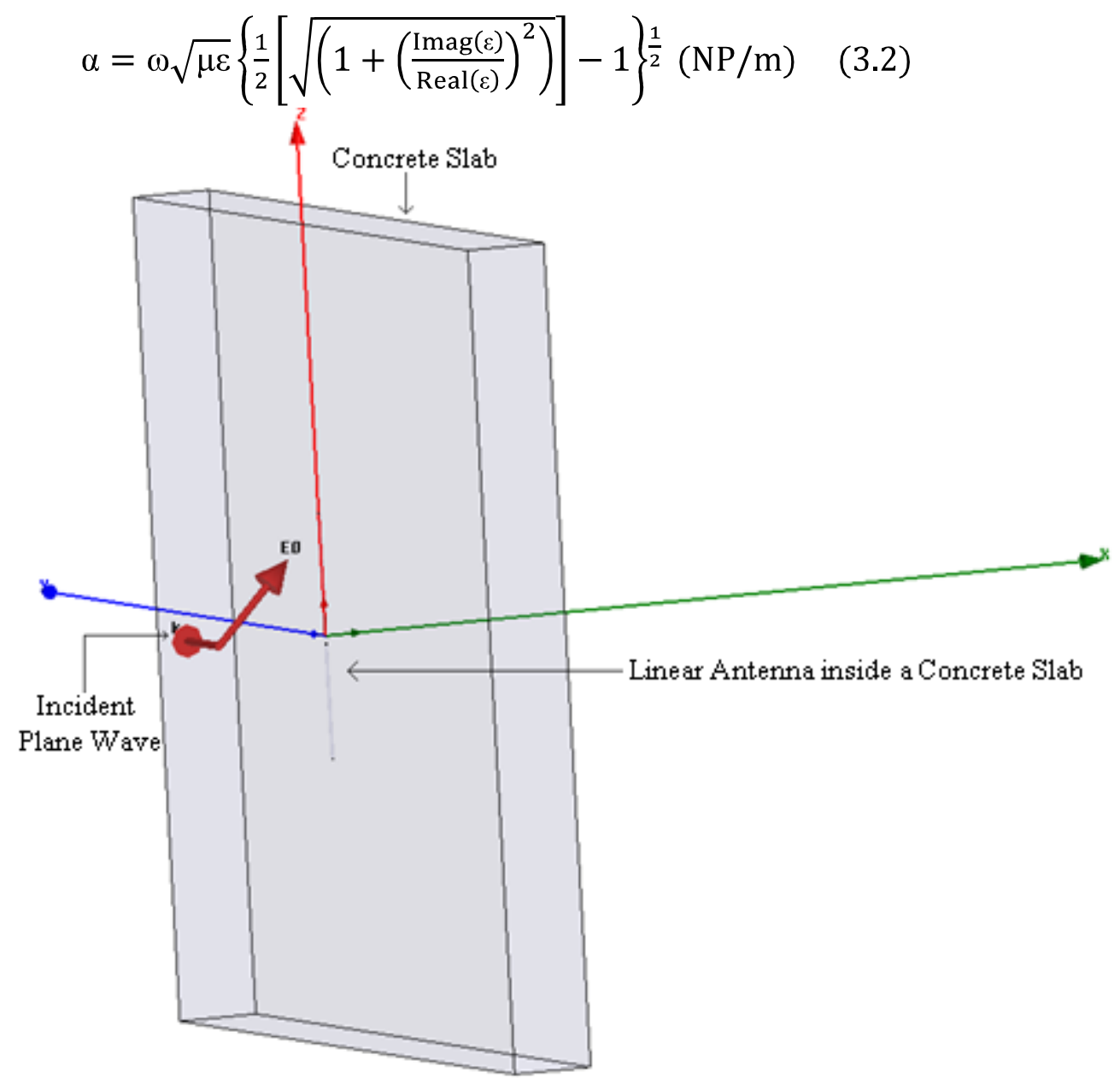

Fig. 3.1 Incident Electric Field on a Concrete Slab 


\subsubsection{Direction of Propagation of a Uniform Plane Wave}

In the above figure, the concrete slab is placed along the z-axis. If the electric field has an $\mathrm{x}$ component, then it would be interpreted as the electric field is incident perpendicular to the concrete slab (Normal Incidence), and if the electric field has a y component, then it would be interpreted as the incident electric field is polarized perpendicular to the concrete slab. Lastly, if the electric field has an x component and a $\mathrm{z}$ component, then it would be interpreted as the incident electric field is polarized parallel to the concrete slab. In fig. 3.1, the electric field has parallel polarization, since it has an $\mathrm{x}$ component as well as a $\mathrm{z}$ component. If $\mathrm{E}_{\mathrm{i}}$ is the incident electric field on a concrete slab, and $E_{t}$ is the transmitted electric field through a concrete slab, then $E_{r}$ is the electric field reflected of the surface of the slab. So the transmitted and incident electric fields for different polarizations can be written as

$$
\begin{gathered}
E_{\mathrm{i}}=\mathrm{a}_{\mathrm{x}} \mathrm{E} ; \mathrm{E}_{\mathrm{t}}=\mathrm{a}_{\mathrm{x}} \mathrm{ETe}^{-\alpha \mathrm{z}} \text { (Normal Incidence) } \\
\mathrm{E}_{\mathrm{i}}=\mathrm{a}_{\mathrm{y}} \mathrm{E} ; \mathrm{E}_{\mathrm{t}}=\mathrm{a}_{\mathrm{y}} \mathrm{ETe}^{-\alpha \mathrm{z}} \text { (Perpendicular Polarization) } \\
\mathrm{E}_{\mathrm{i}}=\left(\mathrm{a}_{\mathrm{x}}-\mathrm{a}_{\mathrm{y}}\right) \mathrm{E} ; \mathrm{E}_{\mathrm{t}}=\left(\mathrm{a}_{\mathrm{x}}-\mathrm{a}_{\mathrm{y}}\right) \mathrm{ETe}^{-\alpha \mathrm{z}} \text { (Parallel Polarization) }
\end{gathered}
$$

Where $\mathrm{T}$ is the transmission coefficient and $\mathrm{z}$ is the depth in a concrete slab. In the above expressions, the phase constant of a concrete slab can also be considered in the exponential term. The Intrinsic Impedance of a concrete slab $\eta_{c}$ is given by

$$
\eta_{c}=\sqrt{\frac{j \omega \mu_{c}}{\sigma_{e f f}+j \omega \varepsilon_{c}}}
$$

$\mu_{c}, \varepsilon_{c}, \sigma_{\text {eff }}$ are the permeability, permittivity and the effective conductivity of a concrete slab respectively. 
The Intrinsic impedance of free space is given by

$$
\eta=\sqrt{\frac{\mu_{0}}{\varepsilon_{0}}}
$$

$\eta, \varepsilon_{0}, \mu_{0}$ are the intrinsic impedance, permittivity and permeability of free space.

The transmission coefficients are given by

$$
\begin{gathered}
\mathrm{T}=\frac{2 \eta_{\mathrm{c}}}{\left(\eta_{\mathrm{c}}+\eta\right)} \text { (Normal Incidence) } \\
\mathrm{T}=\frac{2 \eta_{\mathrm{c}} \cos \left(\theta_{\mathrm{i}}\right)}{\left(\eta_{\mathrm{c}} \cos \left(\theta_{\mathrm{i}}\right)+\eta \cos \left(\theta_{\mathrm{t}}\right)\right)} \text { (Perpendicular Polarization) } \\
\mathrm{T}=\frac{2 \eta_{\mathrm{c}} \cos \left(\theta_{\mathrm{i}}\right)}{\left(\eta \cos \left(\theta_{\mathrm{i}}\right)+\eta_{\mathrm{c}} \cos \left(\theta_{\mathrm{t}}\right)\right)} \text { (Parallel Polarization) }
\end{gathered}
$$

The Average Power Density of the electric field in free space is given by

$$
\mathrm{P}_{\mathrm{i}}=\frac{1}{2} \operatorname{Real}(\mathrm{E} \times \mathrm{H})=\frac{\mathrm{E}^{2}}{2} \operatorname{Real}\left[\frac{1}{\eta}\right]
$$

The Average Power Density of the electric field in a concrete slab is given by

$$
\mathrm{P}_{\mathrm{t}}=\frac{1}{2}(\mathrm{E} \times \mathrm{H})=\frac{|\mathrm{E}|^{2}}{2} \mathrm{e}^{-2 \alpha \mathrm{Z}} \text { Real }\left[\frac{1}{\eta}\right]
$$

\subsection{Power received by an antenna}

Power received by an antenna when it is excited by a plane wave was calculated using the equation

$$
\text { Power Received } \mathrm{P}_{\mathrm{r}}=\frac{\left|\mathrm{V}_{\mathrm{T}}\right|^{2}}{4 \mathrm{R}_{\mathrm{r}}}=\frac{\mid \mathrm{ETe} \mathrm{e}^{-\left.\alpha \mathrm{Z}_{\mathrm{l}}\right|^{2}}}{4 \mathrm{R}_{\mathrm{r}}}
$$


$\mathrm{E}$ is the magnitude of electric field, $\mathrm{T}$ is the transmission coefficient, $\alpha$ is the attenuation, $\mathrm{Z}$ is the depth in the concrete slab, $l$ is the length of the antenna and $R_{r}$ is the radiation resistance of the antenna. Since the material used in this case is a concrete slab, it is essential to use wavelength in a concrete slab rather than wavelength in free space. The wavelength in a concrete slab is given by

$$
\lambda_{\text {concrete }}=\frac{\lambda_{\text {freespace }}}{\sqrt{\mathrm{e}_{\mathrm{r}}}}
$$

The length of the dipole was taken to be $\frac{\lambda}{2}$ for a linear dipole, and for a circular small loop the effective length was used as

$$
\mathrm{L}=\mathrm{K}_{0} \mathrm{~S} \cos (\psi) \sin \left(\theta_{\mathrm{i}}\right)
$$

Where $\mathrm{S}$ is the area of the loop, and the factor $\cos (\psi) \sin \left(\theta_{\mathrm{i}}\right)$ is introduced because the open circuit voltage is proportional to the magnetic flux density, which is normal to the plane of the loop.

\subsection{D Fractal Hilbert Dipole Antennas}

3D-fractal rectangular Koch dipole and 3D fractal Hilbert dipole antennas [60] exhibit lower resonant frequencies and small volume occupations. For a self-similarity fractal, the fractal dimension is given by the equation

$$
\mathrm{D}_{\mathrm{s}}=\frac{\ln (\mathrm{N})}{-\ln (\gamma)}
$$

Where $\mathrm{N}$ is the number of copies of the whole object, and $\gamma$ is the scale factor for each copy.

A fractal-dimension is a measure of the space-filling properties and the complexity of the fractal shape [61]. The two methods that can be used to enhance the design of a fractal antenna are: designing miniaturized antenna elements and designing self similar antenna 
elements. Designing miniaturized antenna elements leads to antennas which are more discrete for the end user. Designing self similar multiband antennas allows a user to integrate several aspects of a system into one antenna. Antennas designed using both these strategies can be incorporated into highly advanced array and smart antenna designs. Usually, the fractal [62] is restricted to $1 \mathrm{D}$ and $2 \mathrm{D}$-space designs. The benefits of space-filling 3D-fractal antenna elements [63] are limited, but it can be efficient in a self-similarity design.

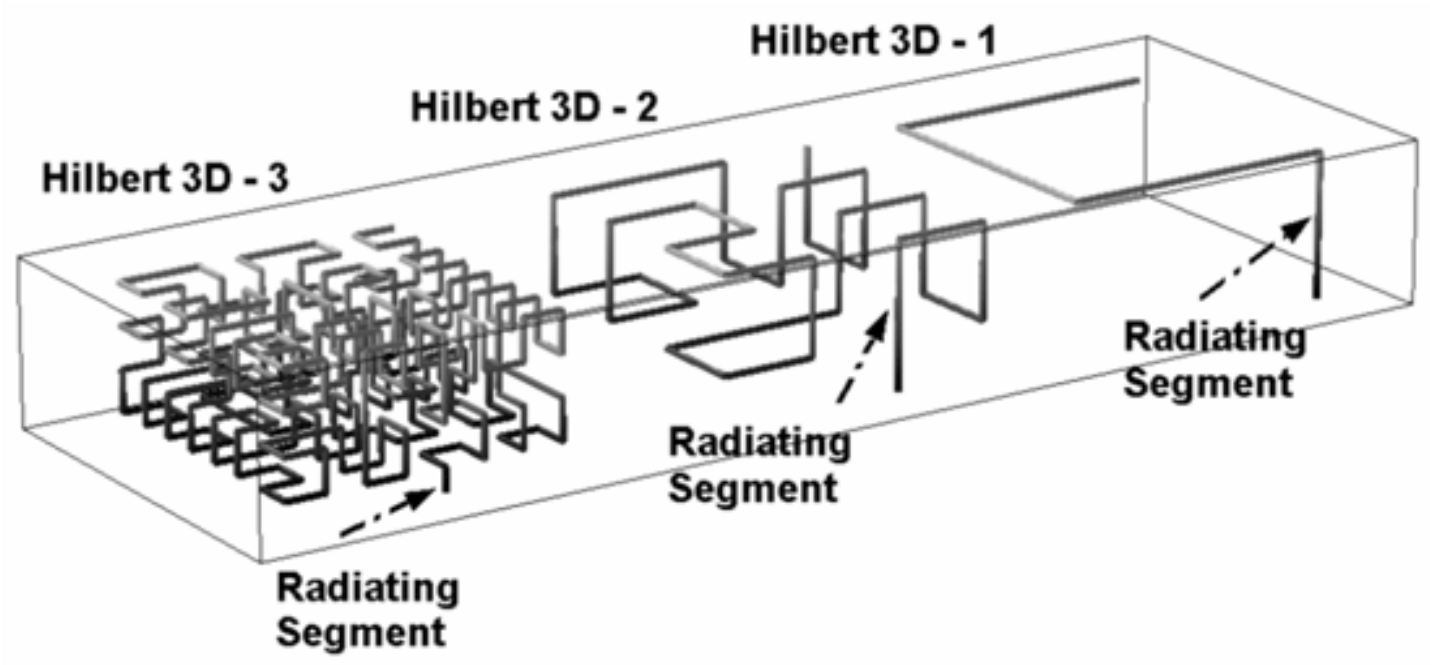

Fig. 3.2 3D Fractal Hilbert Dipole Antenna [18]

The 3D fractal shaped wire antennas can give resonance compression and multiband behavior. The length and the radiation resistance of the 3D fractal Hilbert antenna are given in the table below.

Table . 3 Radiation resistance of a Hilbert 3D-2 Antenna [18]

\begin{tabular}{|c|c|c|}
\hline Antenna & $\begin{array}{c}\text { Total wire length } \\
(\mathrm{cm})\end{array}$ & $\begin{array}{c}\mathrm{R}_{\mathrm{r}} \text { at resonance } \\
(\mathrm{ohms})\end{array}$ \\
\hline Hilbert 3D-2 & 184.99 & 0.16 \\
\hline
\end{tabular}

This antenna has a higher length and a lower radiation resistance. If the power received equation (3.13) in section 3.3 is closely examined, it is observed that a higher length and a lower radiation resistance enable an antenna to receive more power. 


\subsection{Power Accepted}

For a general radiating structure, the accepted power is given by

$$
\mathrm{P}_{\mathrm{acc}}=\frac{1}{2} \operatorname{Real}\left(\mathrm{E} \times \mathrm{H}^{*}\right) \mathrm{ds}
$$

$\mathrm{P}_{\mathrm{acc}}$ is the power accepted in watts and ds is the local port boundary unit. Real is the real part of the complex number. $\mathrm{E}$ is the radiated electric field and $\mathrm{H}^{*}$ is the complex conjugate of the magnetic field $\mathrm{H}$.

For the simple case of an antenna with one lossless port containing a single propagating mode, the above expression reduces to

$$
\mathrm{P}_{\mathrm{acc}}=|\mathrm{a}|^{2}\left(1-\mathrm{S}_{11}^{2}\right)
$$

Where a is the complex modal excitation specified and $S_{11}$ is the single-entry generalized scattering matrix.

\subsection{Simulations and Results}

The first question that has to be answered is whether wireless powering in a concrete slab is possible. If assumed that there is a radiating structure in a concrete slab, then the amount of power entering a concrete slab can be calculated from the incident power and the reflections of a port. The thickness of the concrete slab was taken to be very thin $(5 \mathrm{~mm})$, and another surface which was very close ( $5 \mathrm{~mm}$ from the surface of the concrete slice) was defined as a wave port in Ansoft HFSS v8. The reflections of the surface of the concrete slab have to be plotted to estimate approximately the power that penetrates a concrete slab. The electric field inside the concrete slice for two extreme cases of moisture content $(0.2 \%$ and $12 \%)$ was simulated. The electric field values in the middle of the concrete slice were taken, and its 
propagation through a concrete slab for a depth of $10 \mathrm{cms}$ was calculated using the equation (3.13) in section 3.3. The power received was plotted as a function of frequency for the values of a Hilbert 3D-2 antenna in table . 3 using matlab.

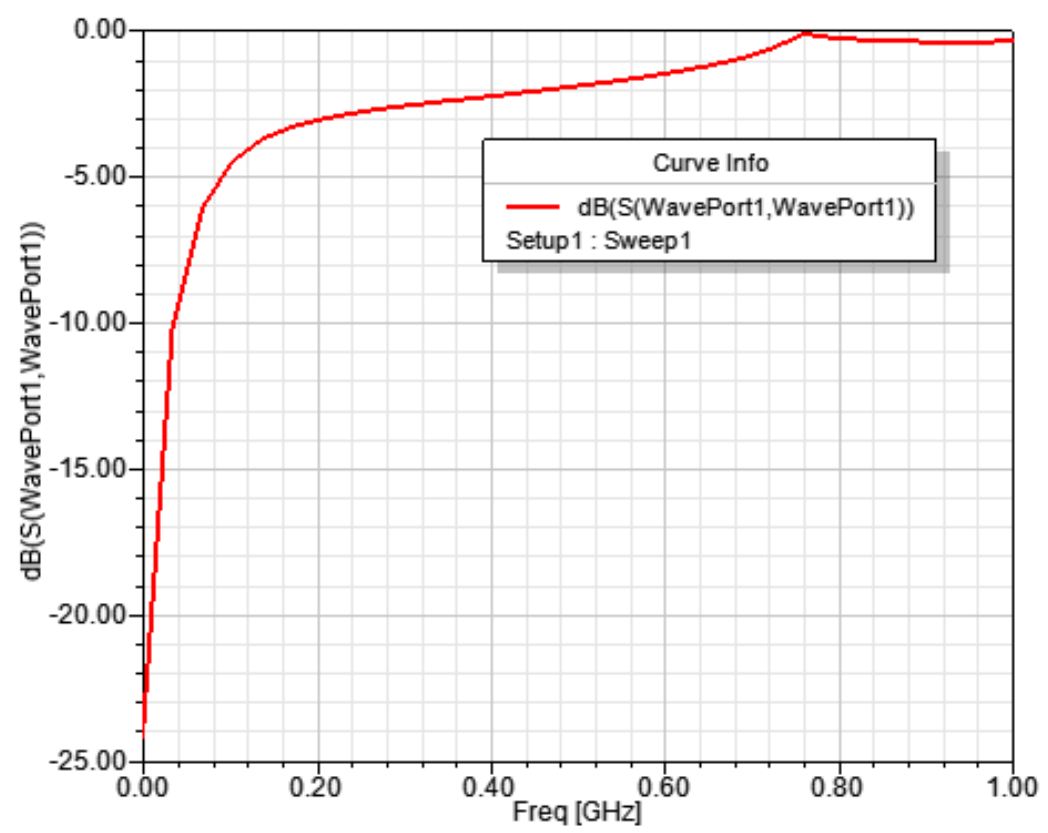

Fig. 3.3 Input Return Loss $\mathrm{S}_{11}(\mathrm{~dB})$ of the surface of the concrete slice

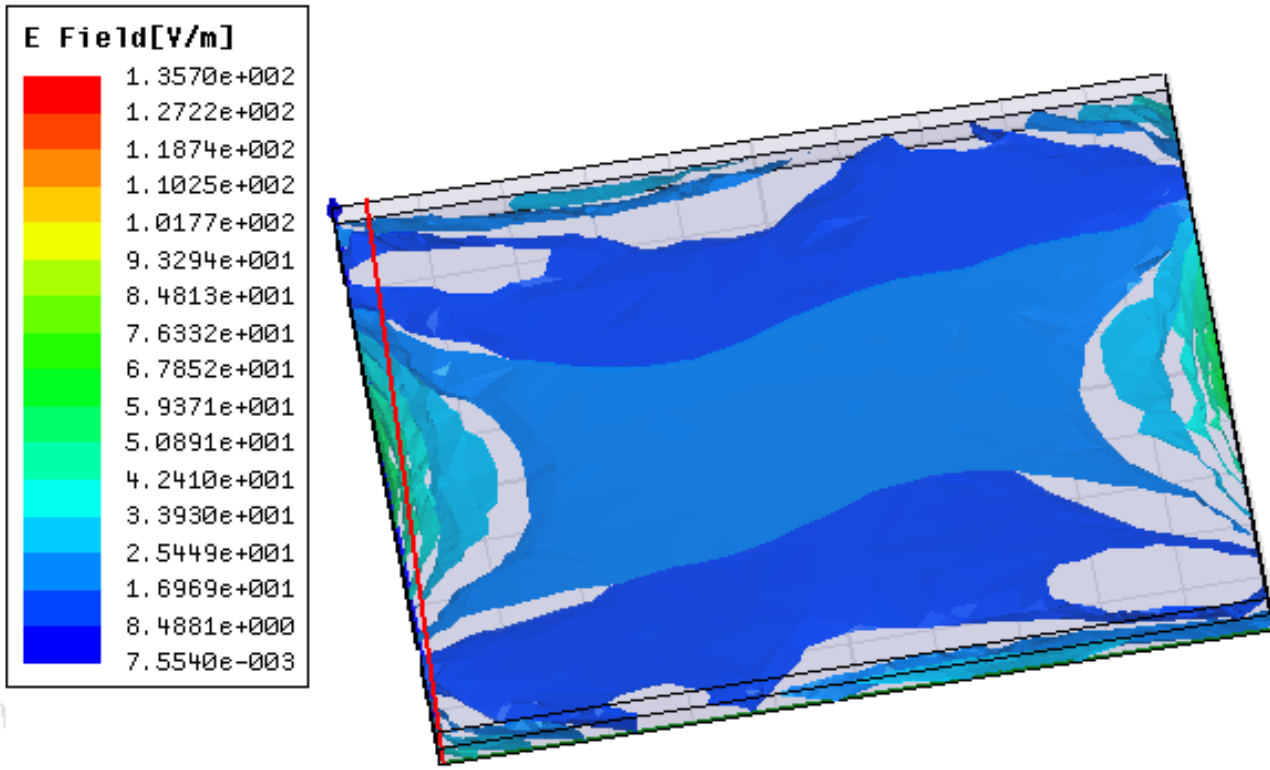

Fig. 3.4 Electric Field inside a concrete slice $(0.2 \%)$ for the incident electric field having X and $\mathrm{Y}$ components 

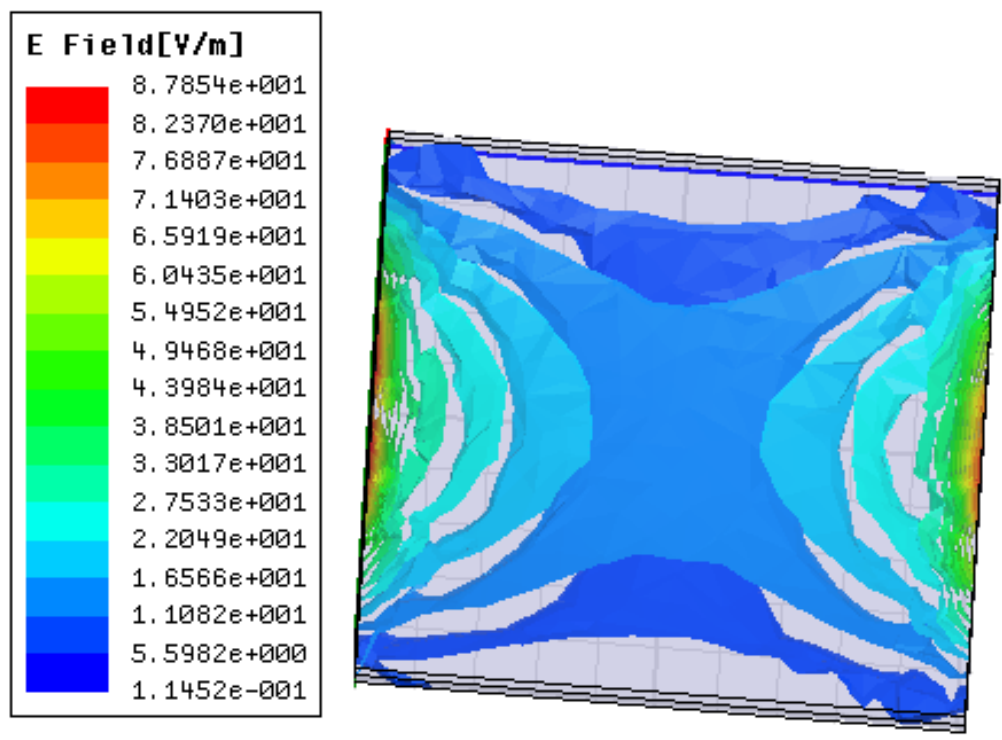

Fig. 3.5 Electric Field inside a concrete slice (12\%) for the incident electric field having X and $\mathrm{Y}$ components
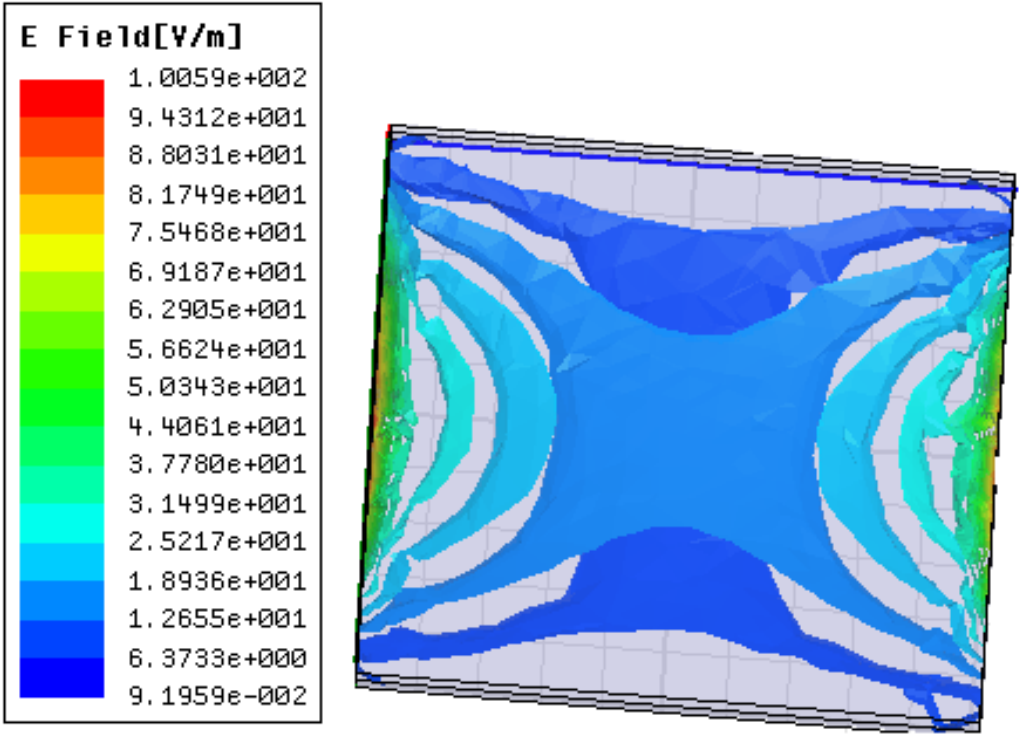

Fig. 3.6 Electric Field inside a concrete slice (12\%) for the incident electric field having an X component 


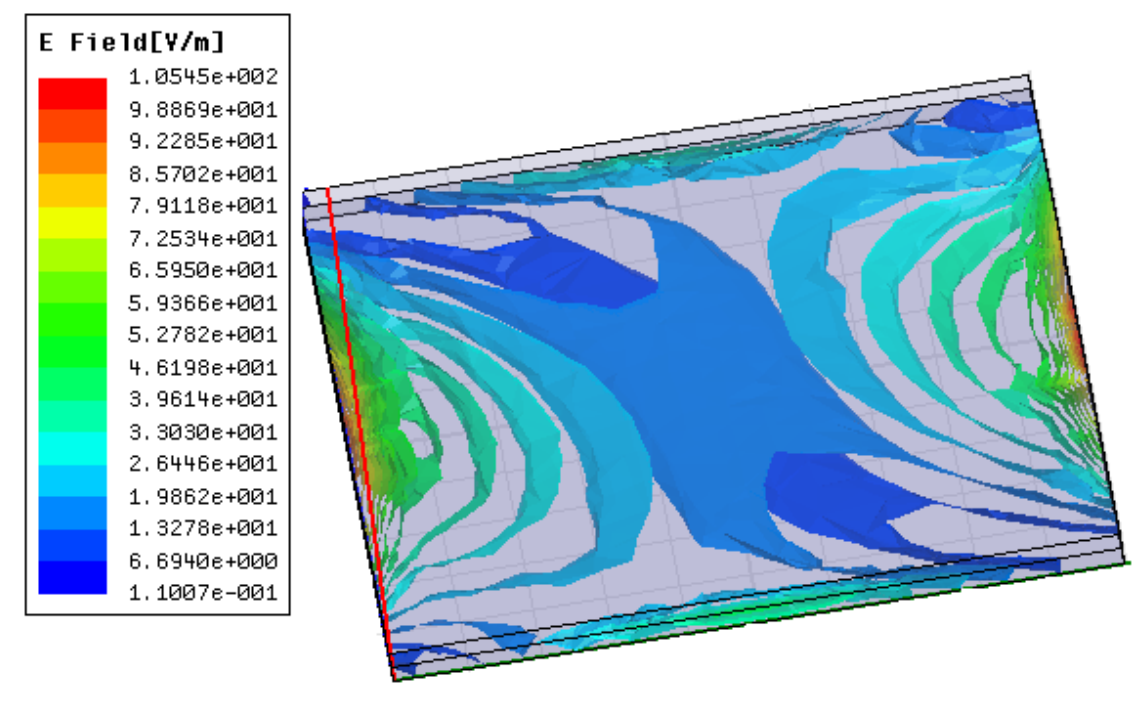

Fig. 3.7 Electric Field inside a concrete slice (0.2\%) for the incident electric field having an $\mathrm{X}$ component

Table . 4 Magnitude of electric field for the four cases [3]

\begin{tabular}{|c|c|l|}
\hline Moisture Content & Component & $\mathrm{E}$ \\
\hline $12 \%$ & $\mathrm{X}$ & 1.2655 \\
\hline $0.2 \%$ & $\mathrm{X}$ & 1.3278 \\
\hline $12 \%$ & $\mathrm{X}$ and $\mathrm{Y}$ & 1.1082 \\
\hline $0.2 \%$ & $\mathrm{X}$ and $\mathrm{Y}$ & 1.6969 \\
\hline
\end{tabular}

The power received by an antenna vs. frequency was plotted neglecting the loss resistance of the antenna for the above four cases, and the behavior was found to be approximately the same.

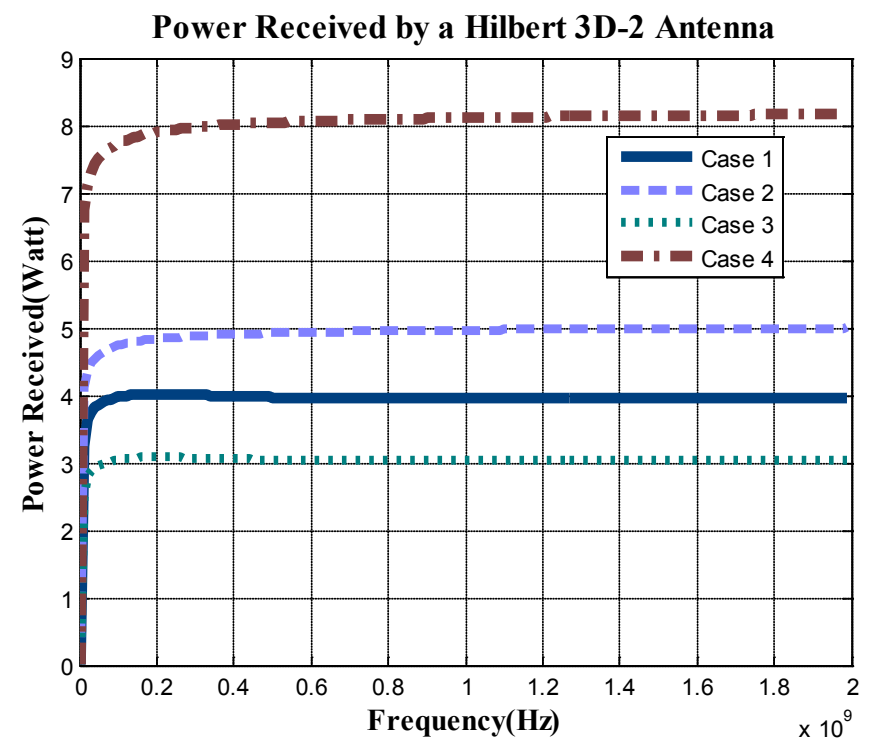

Fig. 3.8 Power received by a Hilbert 3D-2 Antenna for the First Debye model of relative permittivity of concrete 


\subsection{Additional Simulations and Results}

To investigate further the behavior of electric field penetrating a concrete slice, the thickness of the concrete slice was increased to $1 \mathrm{~cm}$ and the simulations were done in Ansoft HFSS v11. The results were found to be the same for a concrete slice with moisture contents $0.2 \%$ and $12 \%$. The reflections of the surface of the concrete slice and the electric field inside the slice were plotted.

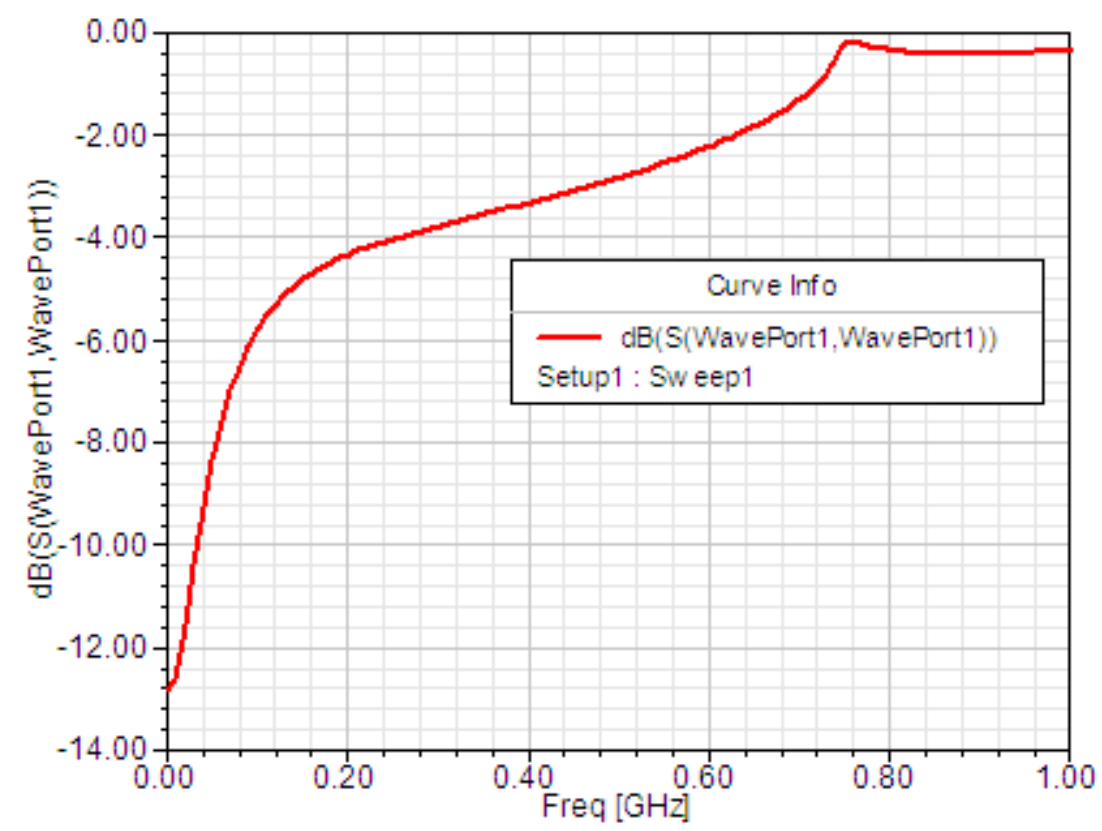

Fig. 3.9 Input Return Loss $\mathrm{S}_{11}(\mathrm{~dB})$ of the surface of the concrete slice
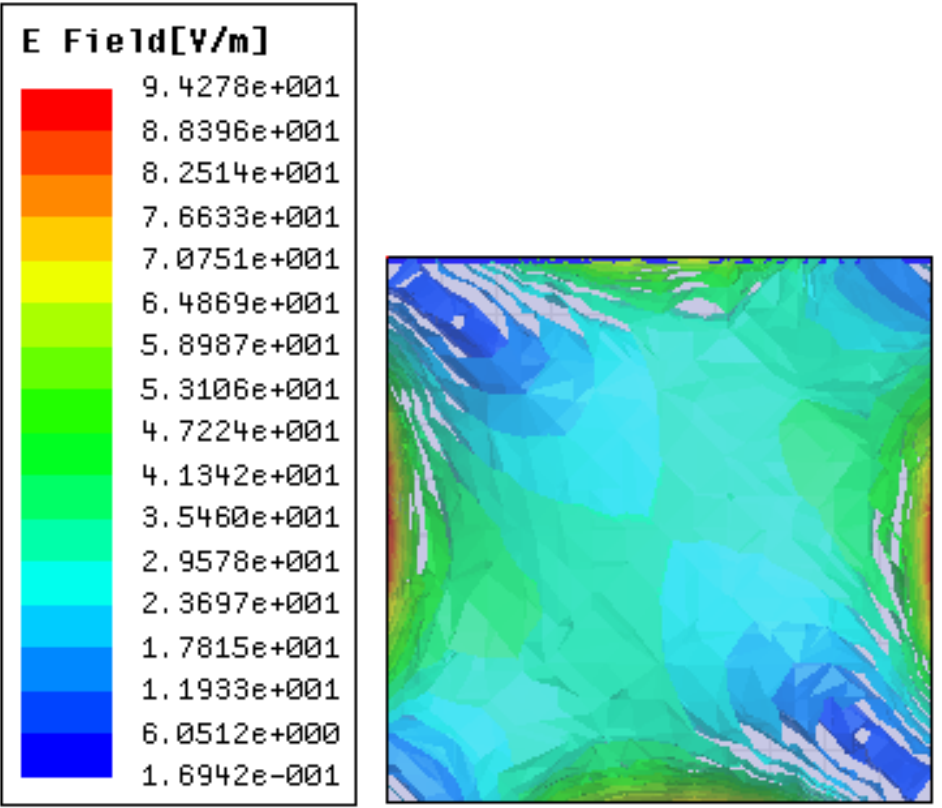

Fig. 3.10 Electric Field inside the concrete slice 


\subsection{Summary}

The reflections of the surface of the concrete slice in fig. 3.3 and fig. 3.9 have shown that it is easier to penetrate a concrete slab at lower frequencies. The electric field plots show sufficient electric field to power a sensor inside a concrete slab. The results from fig. 3.3 and fig. 3.8 have shown that if the Hilbert 3D-2 Antenna is designed such that the resonant frequency is between $40 \mathrm{MHz}$ and $900 \mathrm{MHz}$, then the power received by an antenna would be adequate to power a sensor. The Hilbert 3D-2 antenna used in this research has a resonant frequency of $51.4 \mathrm{MHz}$ which satisfies our cause. As dipoles are becoming obsolete, research has to be done to investigate if microstrip antennas and reconfigurable antennas can be used to enable wireless powering. 


\section{Chapter 4 \\ Reconfigurable Antennas \& Wireless Powering}

\subsection{Introduction}

Sensors that are buried inside a concrete slab for various data collection purposes are usually powered with batteries or wires. The concept of wireless powering arises from the fact that sensors can also be powered from the power delivered to the load resistance of an antenna. As a fractal surface has a larger surface area, it is an ideal choice to wirelessly power a sensor inside a concrete slab. If the antenna is large, then two or more sensors can be powered by a single antenna. Existing reconfigurable antenna designs $[1,2]$ have been used to show that sensors buried inside a concrete slab can be powered wirelessly using reconfigurable antennas.

A new reconfigurable planar inverted fractal antenna design that improves performance and enables wireless powering will be shown in this chapter. The concepts of the Reconfigurable Planar Inverted Fractal antenna (RPIFA) and the Reconfigurable Sierpinski Gasket Antenna (RSGA) explained in sections 2.9.1 and 2.9.2, have been merged to create a Reconfigurable Planar Inverted Sierpinski Gasket Fractal Antenna (RPISGFA). The antenna buried inside a concrete slab has to absorb power from the plane wave incident on the concrete slab. Any device in free space can be powered from the RF energy that its antenna receives from another antenna. Sensors in free space and in a concrete slab can be powered based on the electric field incident on the surface of the receiving antenna. The concept of self similarity in antennas, which increases the length of that part of the antenna that is capable of receiving electromagnetic energy inside a concrete slab, is demonstrated in this chapter.

\subsection{Reconfigurable Antennas vs. Dipoles for Wireless Powering}

A reconfigurable patch antenna has switches which enable it to stop a particular part of the antenna from radiating. A microstrip patch antenna does not have switches, and thus cannot 
stop any part of itself from radiating. In the transmitting mode, the switches in a reconfigurable patch antenna can be used as points where excitation can be applied. Similarly, in the receiving mode, coaxial lines can be applied along with the switches to extract the received signal. So when a reconfigurable patch antenna is compared to a microstrip patch antenna or a dipole antenna, the first noticeable difference is that the reconfigurable patch antenna has more excitation points.

As shown in fig. 2.12, when an antenna receives radio frequency energy from another antenna, it has only one excitation point where the coaxial lines receive the power delivered to the antenna. If the receiving antenna in fig. 2.12 is replaced with a reconfigurable antenna such as the RPIFA shown in section 2.8.2, then multiple receiving coaxial lines can be used to power multiple sensors. An antenna with one receiving coaxial line will have high reflections as it is receiving the entire power incident on the antenna. The presence of multiple coaxial lines will decrease the number of reflections when an antenna receives power from another antenna. Thus, reconfigurable antennas can be used to enable wireless powering.

\subsection{Reconfigurable Planar Inverted Sierpinski Gasket Fractal Antenna (RPISGFA)}

In order to understand how the RPISGFA was created, it is necessary to understand the Planar Inverted Fractal Antenna (PIFA), and the concept of self-similarity of a Sierpinski Gasket Antenna. A Planar Inverted Fractal Antenna is considered to be an Inverted Fractal antenna with the wire radiator element replaced by a planar radiating element to increase the bandwidth. A Planar Inverted Fractal Antenna [64] consists of three elements: a rectangular planar element located parallel and above a ground plane, a plate or a pin that short circuits the radiating element and the ground plane, and an excitation mechanism for the radiating element.

The radiating element is placed parallel to and above the ground plane [64] to reduce the height of the antenna while maintaining a resonant trace length. Capacitance introduced to the input impedance of the antenna by the parallel section is compensated by implementing a 
short circuiting pin. Excitation current to the radiating element is divided between the radiating element and the ground plane. Since the excitation current is divided between the radiating element and the ground plane, the resulting electromagnetic field of the antenna [64] is a joint field of the radiating element and the ground plane. This antenna functions as a perfect energy reflector [64] when the dimensions of the ground plane are very large (or close to infinity). The PIFA antenna has reduced backward radiation toward the user's head, thus minimizing the electromagnetic wave power absorption (SAR). Since the PIFA antenna has moderate to high gains in both states [64] of vertical and horizontal polarization, it is extensively used in wireless communications where the orientation of the antenna varies, and in an environment where large amount of reflections are present. In such situations, the total field is the vector sum of horizontal and vertical states of polarization. The radiation pattern of the PIFA antenna is the [64] relative distribution of radiated power as a function of direction in space, and is determined in the far-field region as a function of directional coordinates. Power flux density, field strength, phase, and polarization are the radiation properties of the PIFA antenna.

An object is said to be self similar [65], if it is exactly or approximately similar to a part of itself. Self similarity is a typical property of fractals. Scale invariance is a form of selfsimilarity, where at any magnification [65] there is a smaller piece of the object that is similar to the whole. This property of scale invariance and self similarity has to be used to design an antenna which can enable many applications in the $3-30 \mathrm{GHz}$, stated earlier in table . 2. In order to convert the existing Sierpinski gasket antenna into a Reconfigurable Planar Inverted Sierpinski Gasket Fractal Antenna, four additional switches were added. The two bottom radiating triangles are connected to the top radiating triangle by two switches, and four other switches connect the antenna to the ground plane. Excitation was applied at the top vertex of the top triangle as shown in fig. 4.1. One of the switches that connects the radiating part to the ground plane can be used to short the planar element to the ground plane. When all the three 
triangles radiate, it is considered to be the first iteration, and when only the first triangle radiates, it is considered to be the second iteration.

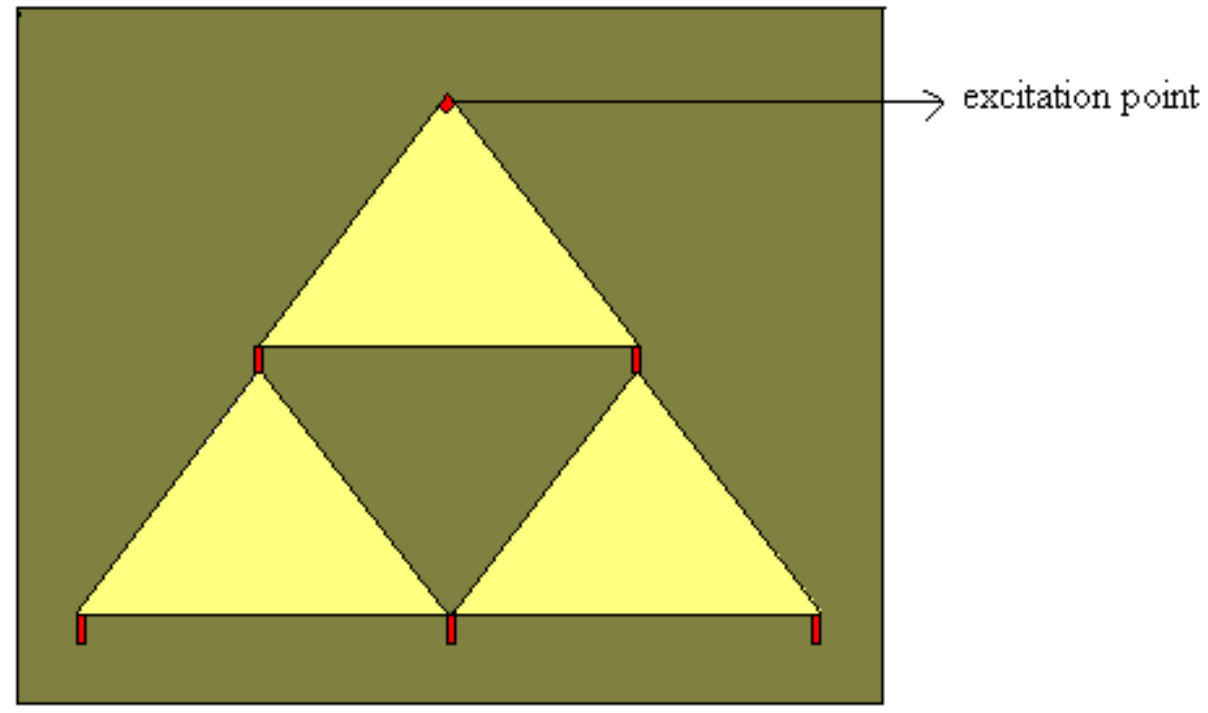

Fig. 4.1 RPISGFA

\subsection{Simulations and Results of the RPISGFA}

The base of the entire Sierpinski triangle is $27.28 \mathrm{~mm}$, and the height is $47.35 \mathrm{~mm}$. The dimensions of the ground plane are $80 \mathrm{~mm} \times 75 \mathrm{~mm}$. A slot was attached to the top triangle, and one face of the slot was assigned as a wave port in Ansoft HFSS v11. The dimensions of the slot are $11 \mathrm{~mm} \times 5 \mathrm{~mm}$. In the second iteration, the switches that connect the radiating triangles to the ground plane were also switched off to make sure that only the top triangle was radiating. The thickness of the ground plane and the radiating part was taken be $0.017 \mathrm{~mm}$. The switches that connect the antenna to the ground plane were simulated as perfect electric conductors, and copper material was used for switches that connect the top triangle to the bottom triangles. Since the switches that connect the antenna to the ground plane were simulated as perfect electric conductors, it can be assumed, for this case, that all the switches are shorting the radiating triangles to the ground plane. The input return $\operatorname{loss}\left(\mathrm{S}_{11}\right)$, VSWR and radiation pattern were plotted for this antenna, when the all three triangles were radiating, and only the top triangle was radiating. 


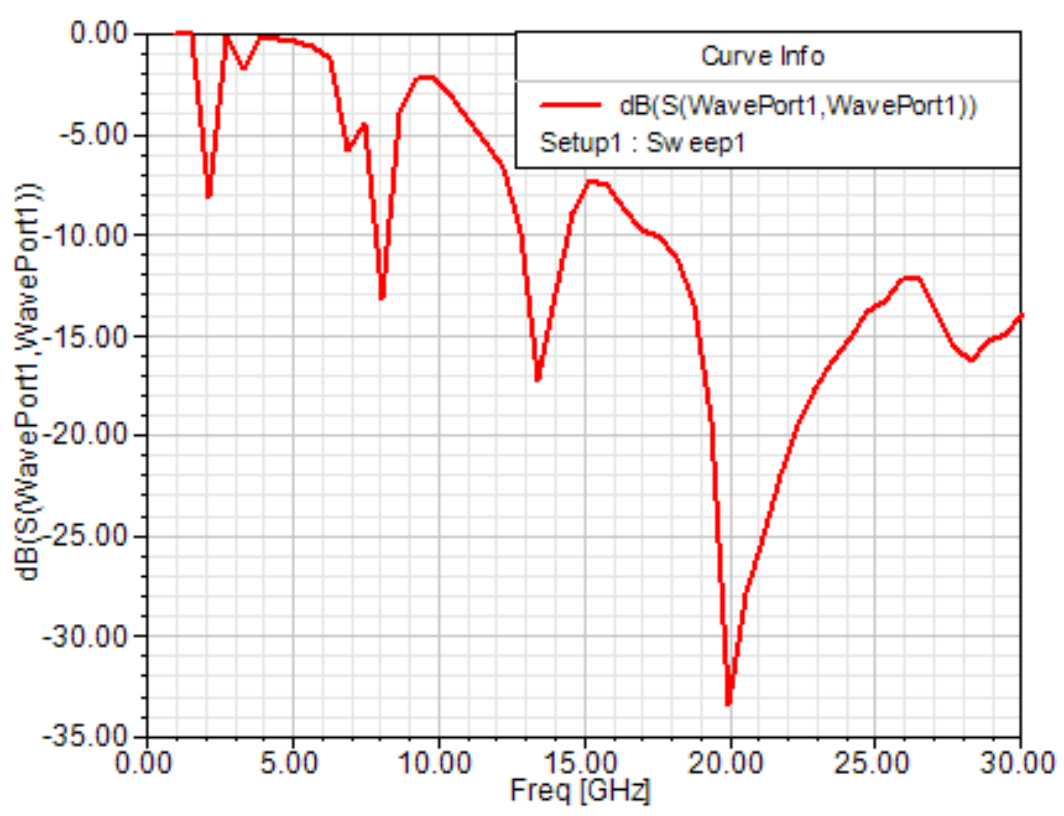

Fig. 4.2 Input Return Loss $\left(\mathrm{S}_{11}\right)$ for the first iteration

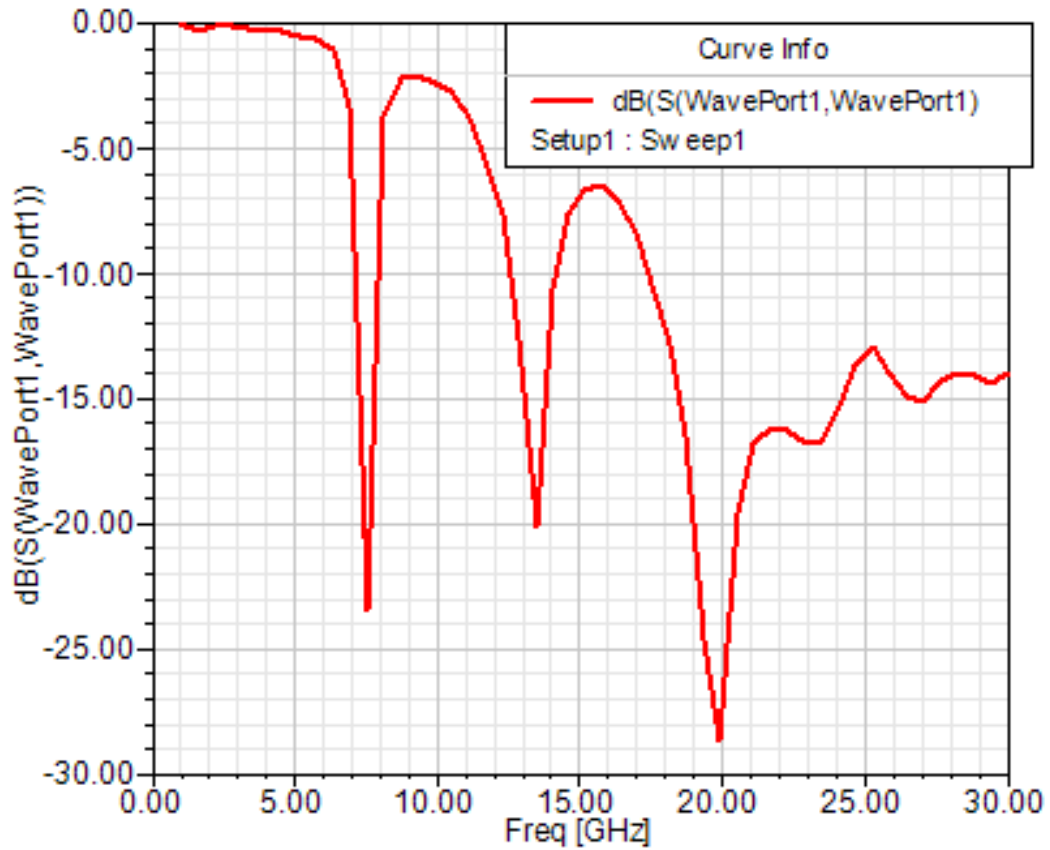

Fig. 4.3 Input Return Loss $\left(\mathrm{S}_{11}\right)$ for the second iteration 


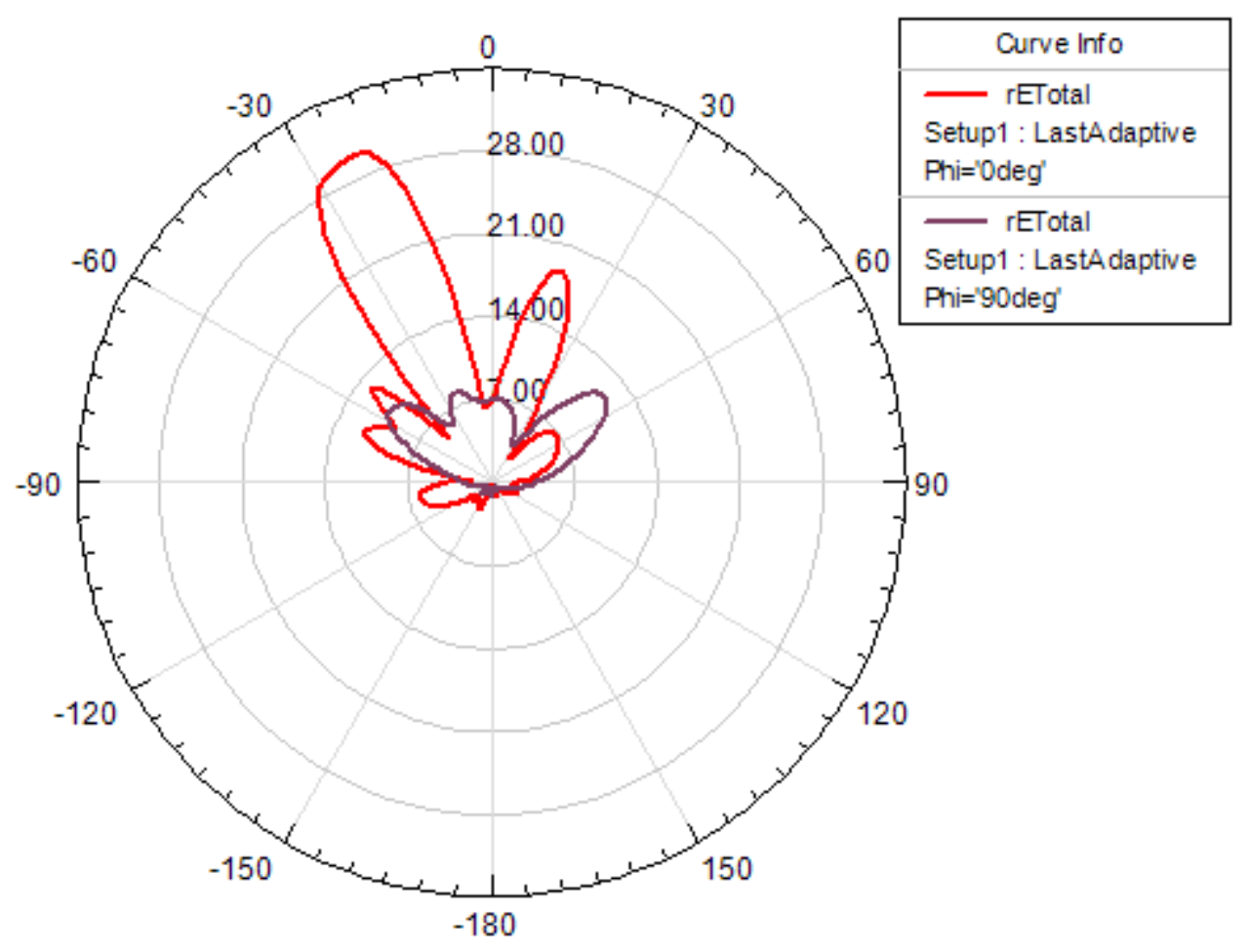

Fig. 4.4 Radiation Pattern for the first iteration

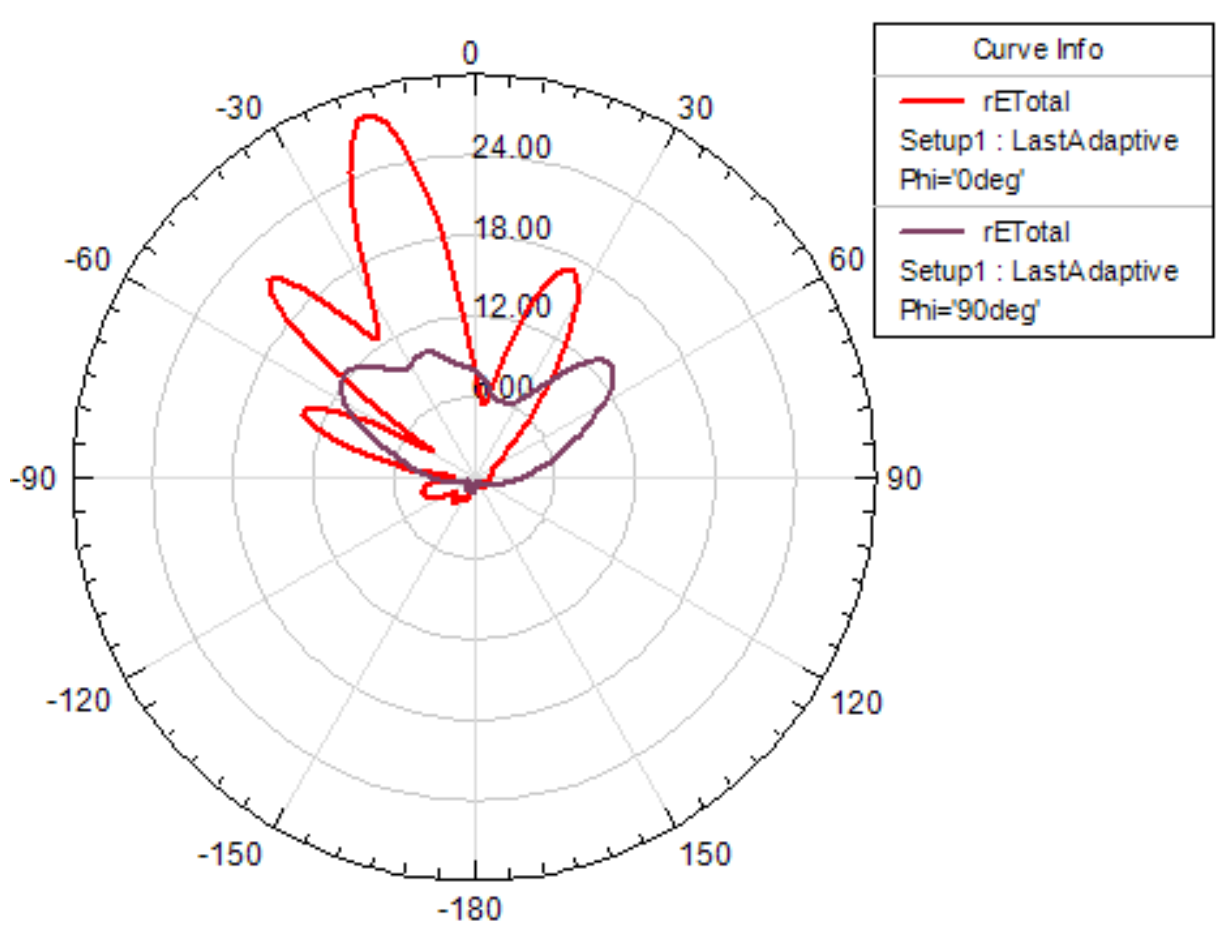

Fig. 4.5 Radiation Pattern for the second iteration 


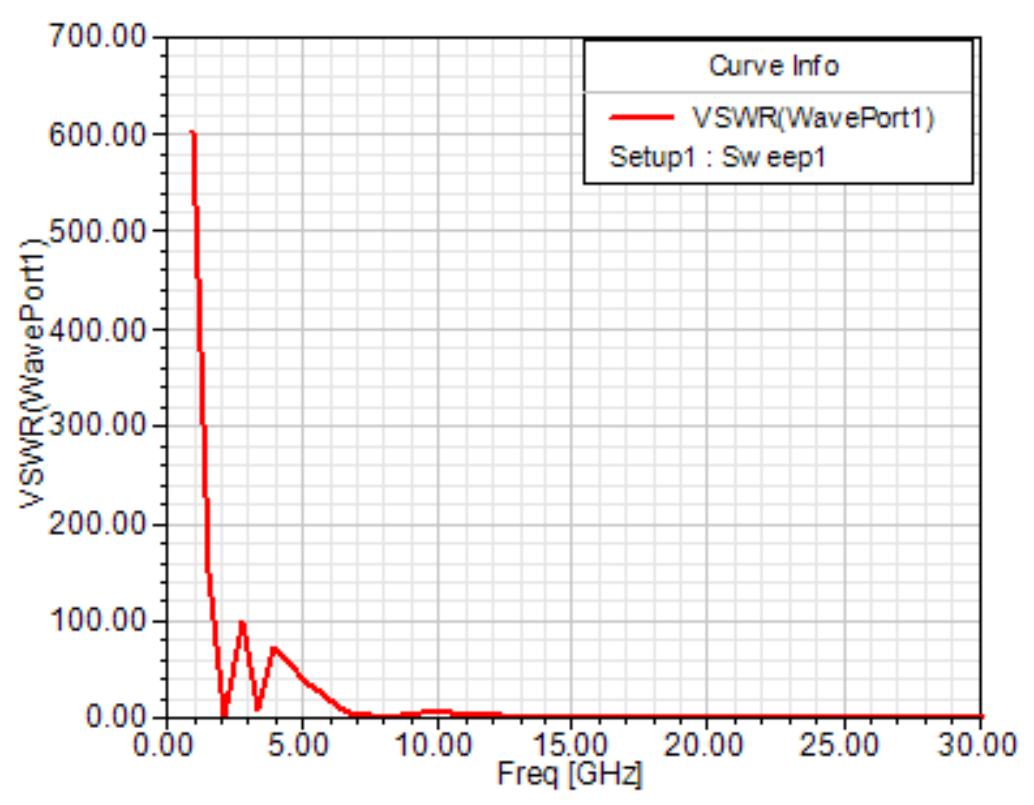

Fig. 4.6 VSWR for the first iteration

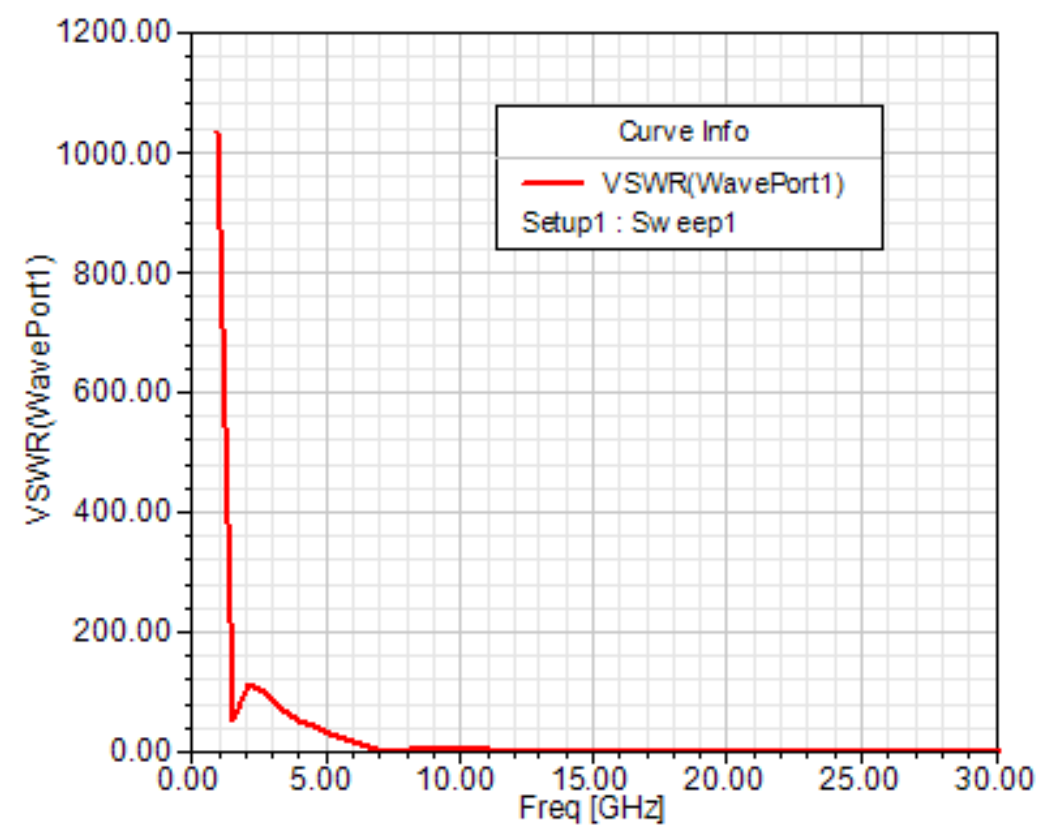

Fig. 4.7 VSWR for the second iteration 


\subsection{Data Analysis}

When the input return losses of the RPISGFA and the RSGA in section 2.9.1 are compared, remarkable improvements in the performance of the RPISGFA is observed. The RPISGFA has three resonating frequencies in both the iterations. The antenna in both the iterations has very low input return loss from $19 \mathrm{GHz}$ to $30 \mathrm{GHz}$. The radiation patterns have two main lobes and many side lobes. The VSWR is very high until $11 \mathrm{GHz}$ and $7 \mathrm{GHz}$ for the first iteration and the second iteration respectively. The RPISGFA can be used for most of the applications that use the frequency range of 3 to $30 \mathrm{GHz}$.

\subsection{Simulations and Results of the RPISGFA in a concrete slab}

The antenna was kept $12 \mathrm{~cm}$ inside a concrete slab, excited by a plane wave source 10 $\mathrm{cm}$ above the surface of the concrete slab in Ansoft HFSS v11. The reflections of the surface of the concrete slab were plotted as the input return loss as a function of frequency for a concrete slab having $0.2 \%$ and $12 \%$ moisture content. The electric fields on the surface of the antenna for a concrete slab having $0.2 \%$ and $12 \%$ moisture content were also plotted. In this case, all the switches were simulated as perfect electric conductors.

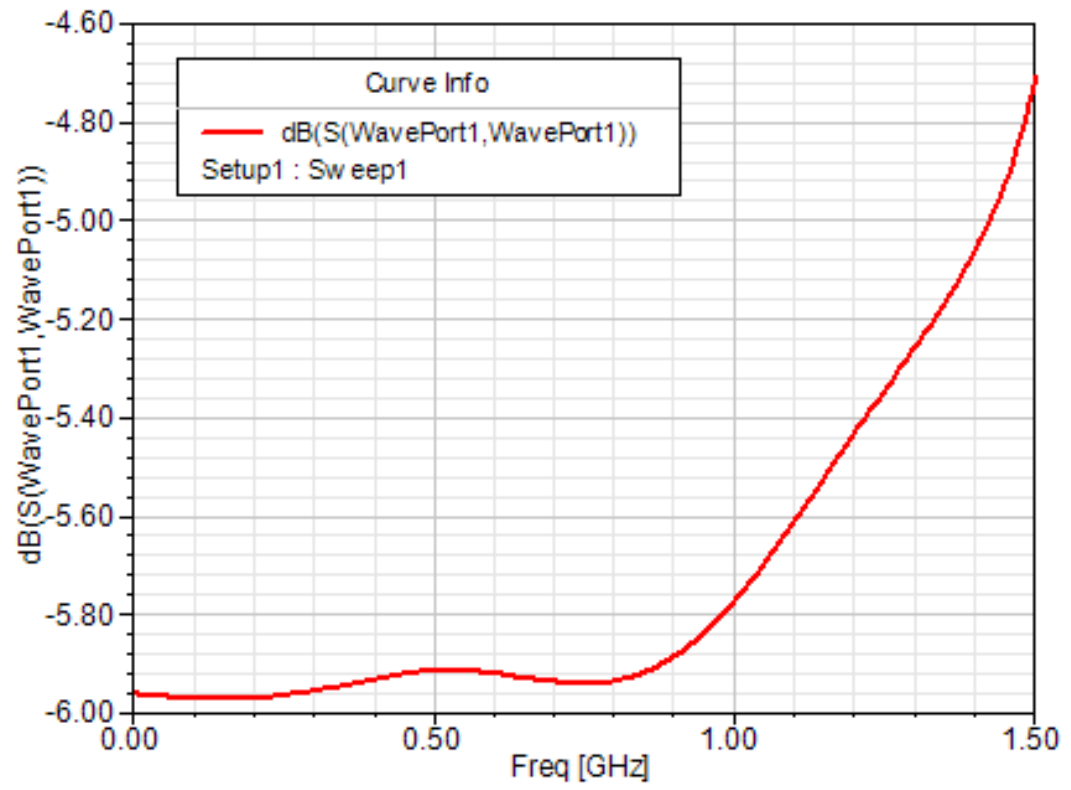

Fig. 4.8 Input Return Loss $\left(\mathrm{S}_{11}\right)$ for a concrete slab (0.2\% moisture content), having the RPISGFA $12 \mathrm{~cm}$ inside 


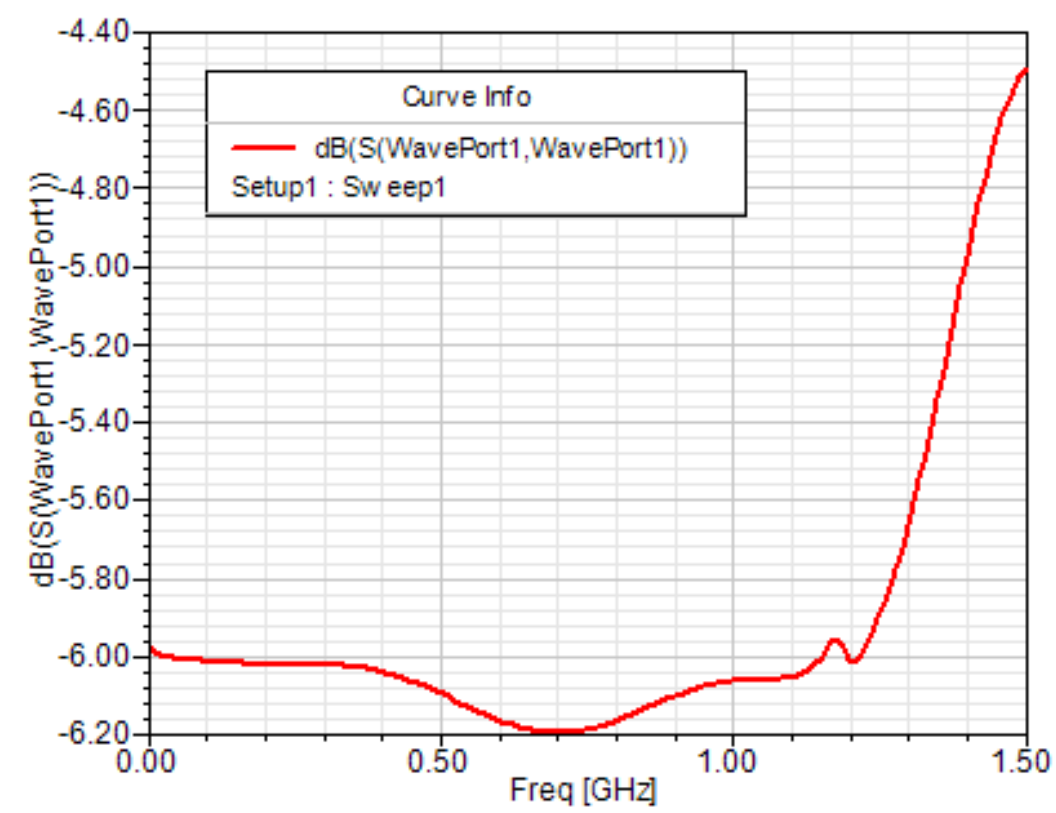

Fig. 4.9 Input Return Loss $\left(\mathrm{S}_{11}\right)$ for a concrete slab (12\% moisture content), having the RPISGFA $12 \mathrm{cms}$ inside it
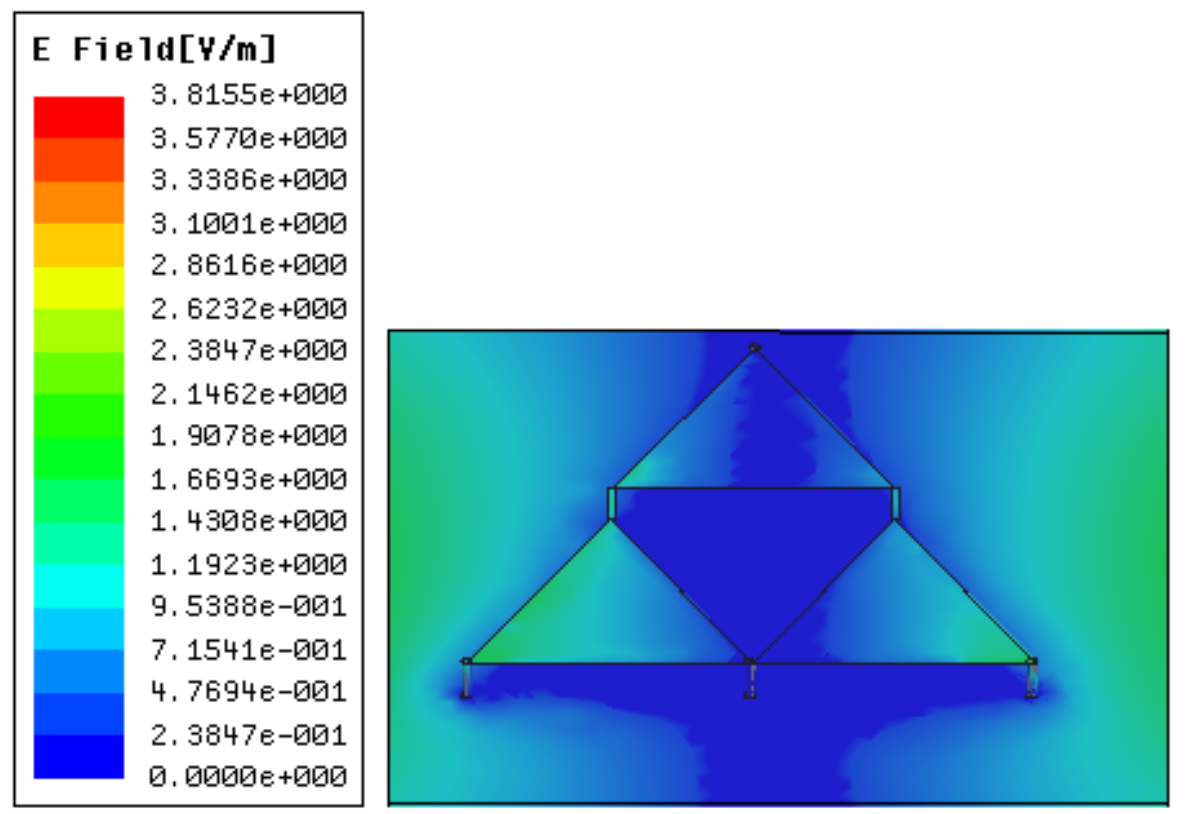

Fig. 4.10 Electric field on the surface of the RPISGFA buried inside a concrete slab having $0.2 \%$ moisture content 


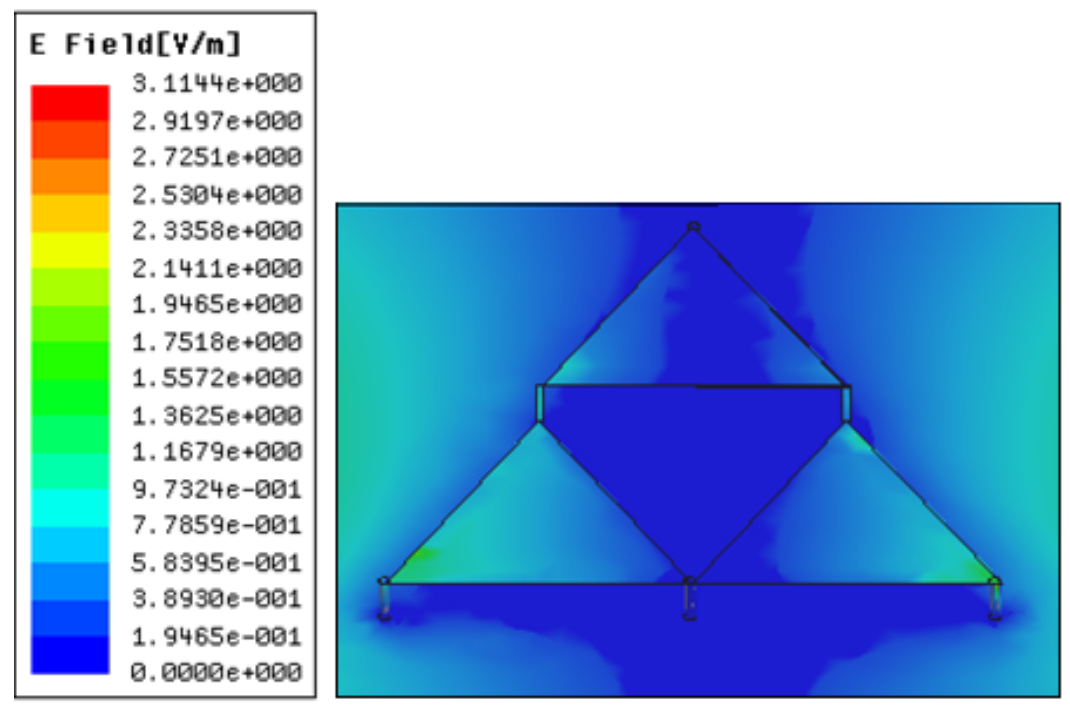

Fig. 4.11 Electric field on the surface of the RPISGFA buried inside a concrete slab having $12 \%$ mo isture content

\subsection{Additional Simulations and Results of the RPISGFA in a concrete slab}

The antenna was also kept $10 \mathrm{~cm}$ inside a concrete slab, excited by a plane wave source $10 \mathrm{~cm}$ above the surface of the concrete slab in Ansoft HFSS v11. The reflections of the surface of the concrete slab were plotted as the input return loss as a function of frequency for a concrete slab having $0.2 \%$ and $12 \%$ moisture content. The electric fields on the surface of the antenna for a concrete slab having $0.2 \%$ and $12 \%$ moisture content were also plotted.

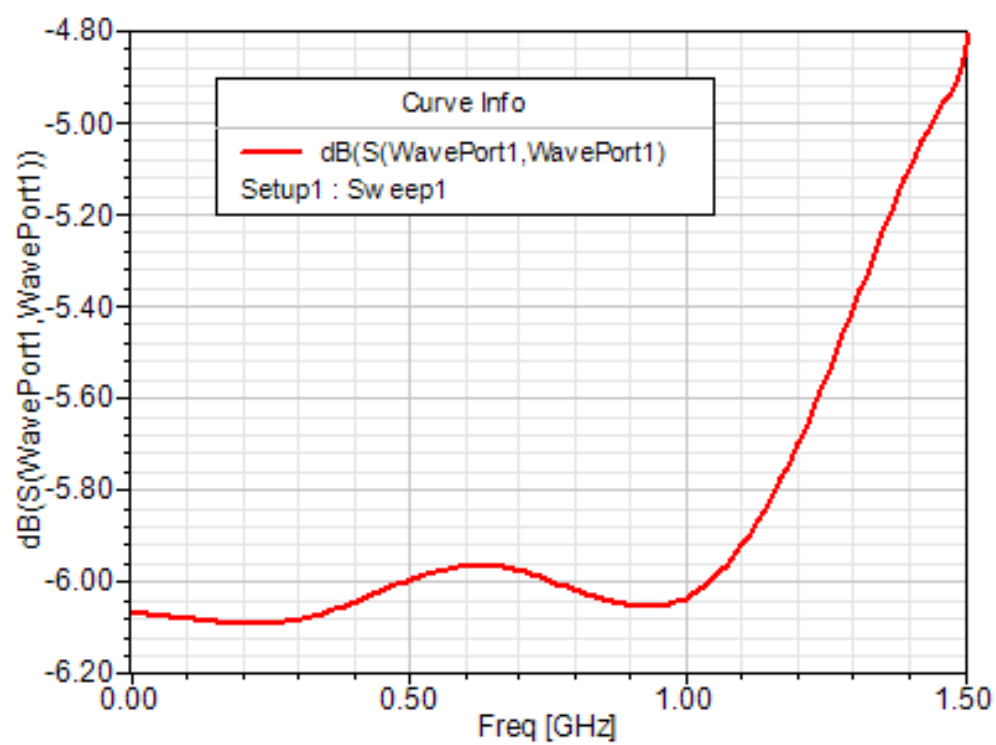

Fig. 4.12 Input Return Loss $\left(\mathrm{S}_{11}\right)$ for a concrete slab ( $0.2 \%$ moisture content), having the RPISGFA $10 \mathrm{~cm}$ inside it 


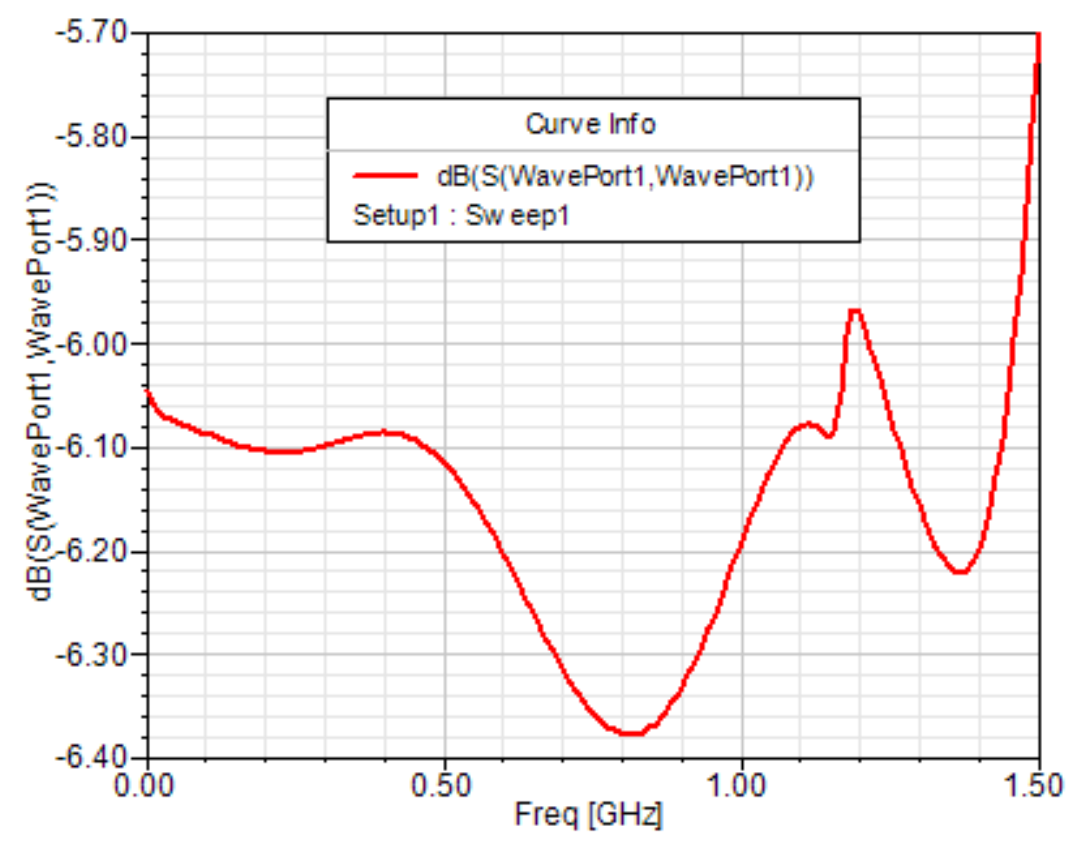

Fig. 4.13 Input Return Loss $\left(S_{11}\right)$ for a concrete slab (12\% moisture content), having the RPISGFA $10 \mathrm{~cm}$ inside it
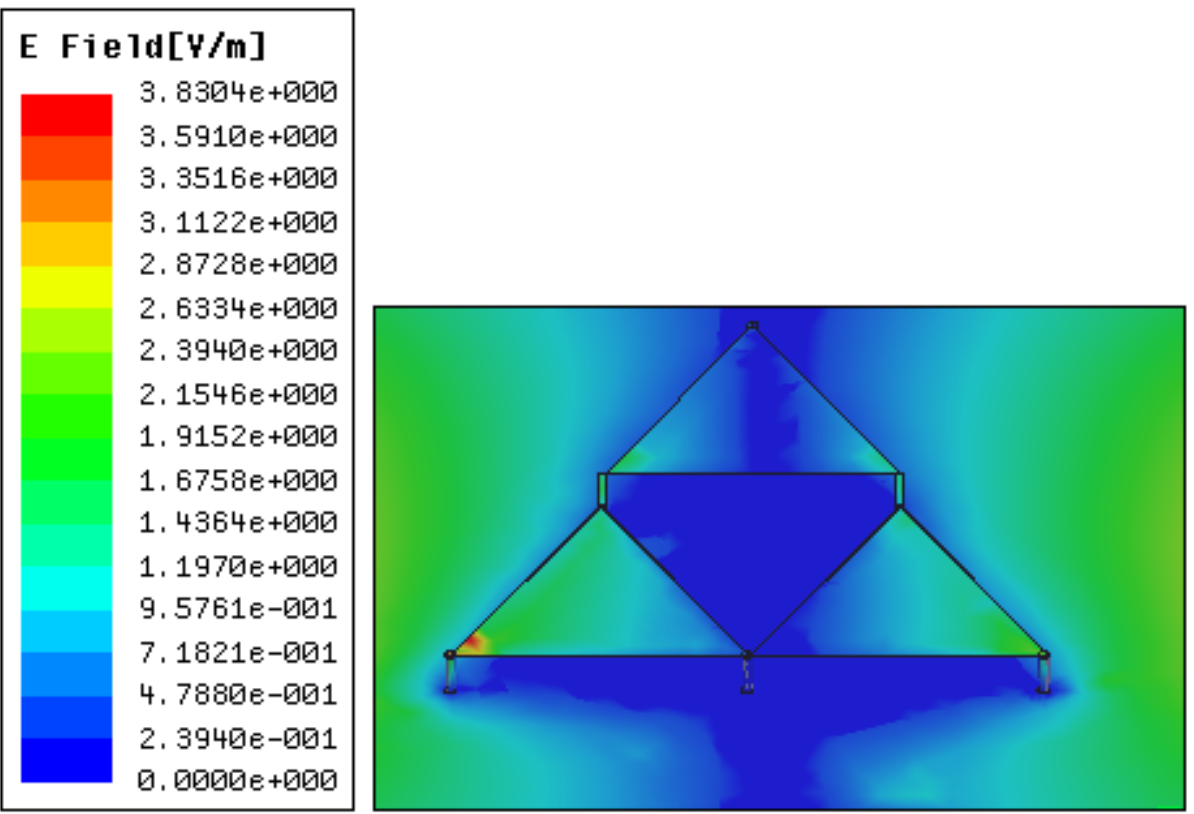

Fig. 4.14 Electric field on the surface of the RPISGFA buried inside a concrete slab having $0.2 \%$ moisture content 


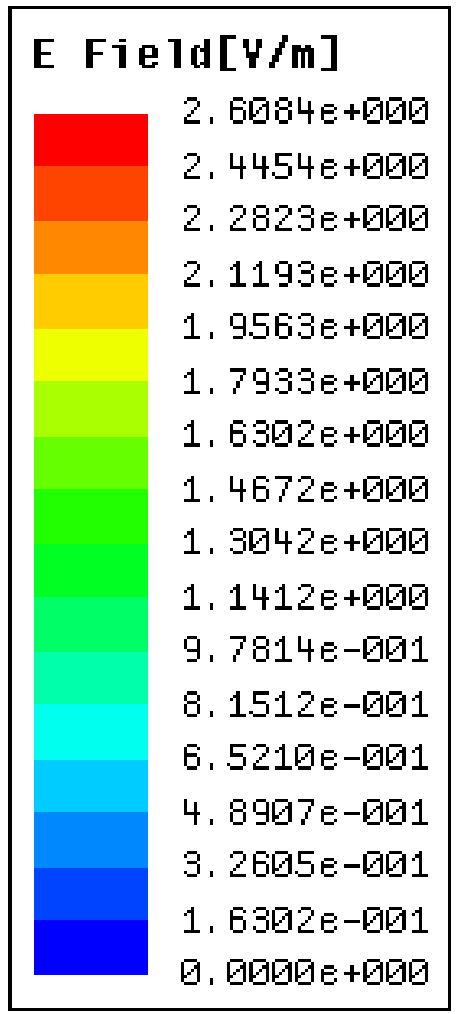

Fig. 4.15 Electric field on the surface of the RPISGFA buried inside a concrete slab having $12 \%$ moisture content

\subsection{Data Analysis}

The data of reflections from the concrete slab in figures 4.8, 4.9, 4.12 and 4.13 show that the best frequency range to penetrate the concrete surface would be from $700 \mathrm{MHz}$ to 900 MHz. The electric fields on the surface of the RPISGFA are high enough to power a sensor such as the temperature sensor or the humidity sensor inside a concrete slab.

\subsection{Simulations and Results of the RPIFA}

The RPIFA described in section 2.9.2 was also kept $12 \mathrm{~cm}$ inside a concrete slab, excited by a plane wave source $10 \mathrm{~cm}$ above the surface of the concrete slab, in Ansoft HFSS v11. The electric fields on the surface of the antenna for a concrete slab having $0.2 \%$ and $12 \%$ moisture content were also plotted. 


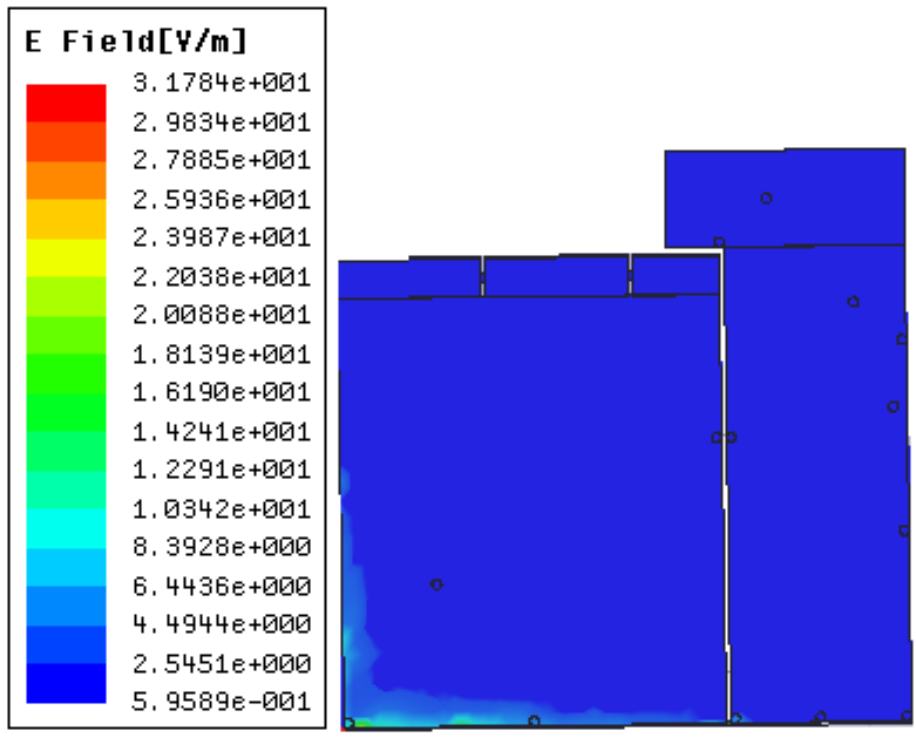

Fig. 4.16 Electric field on the surface of the RPIFA buried inside a concrete slab having 0.2 $\%$ moisture content

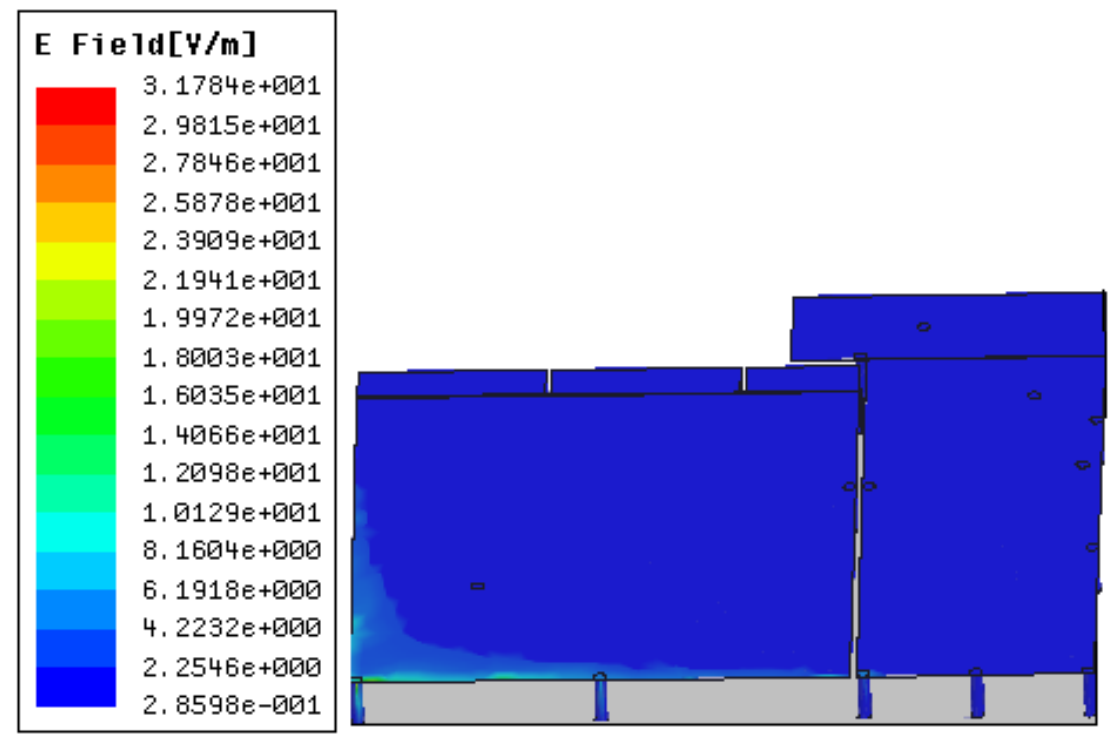

Fig. 4.17 Electric field on the switches of the RPIFA buried inside a concrete slab having 0.2 $\%$ moisture content 


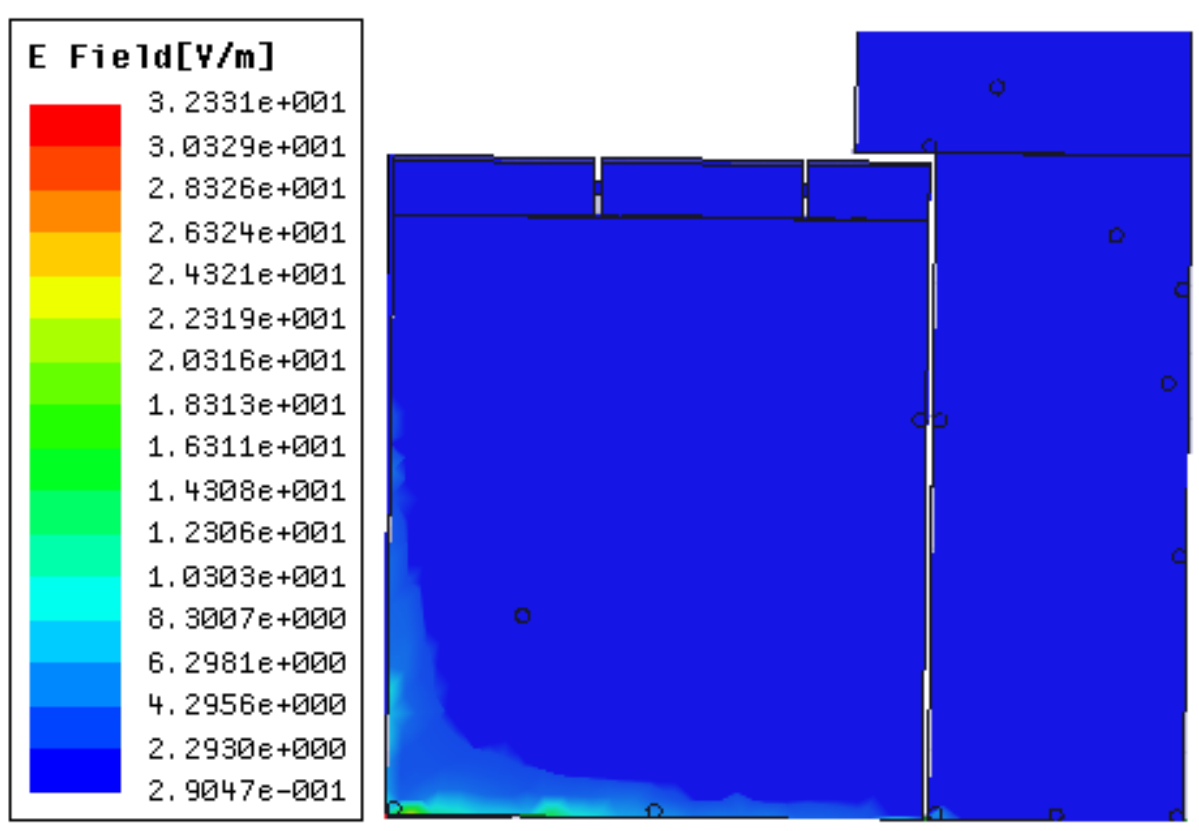

Fig. 4.18 Electric field on the surface of the RPIFA buried inside a concrete slab having 12 $\%$ moisture content
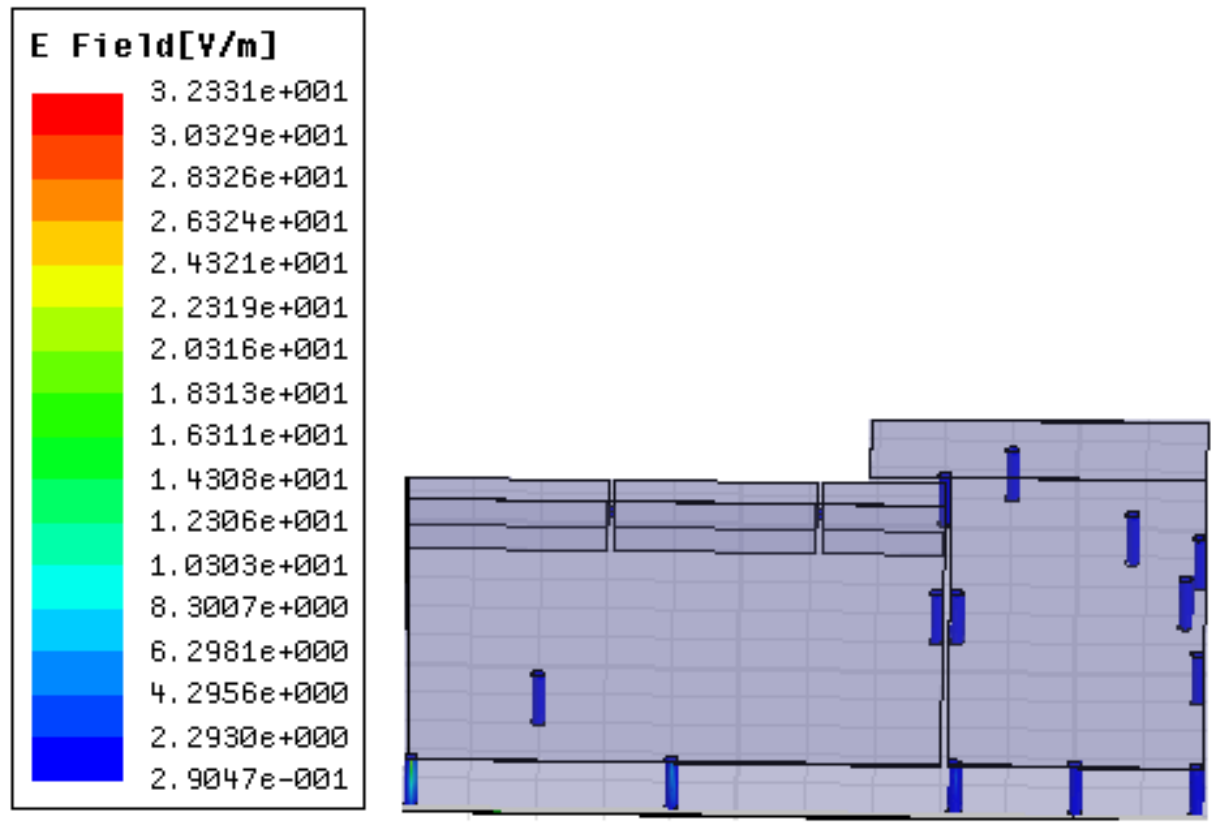

Fig. 4.19 Electric field on the switches of the RPIFA buried inside a concrete slab having 12 $\%$ moisture content 


\subsection{Summary}

The RPISGFA has very low input return losses and can be used to enable many applications which require an antenna with good performance. The above results of the RPIFA and the RPISGFA in a concrete slab confirm that reconfigurable antennas are very good to enable wireless powering. High electric fields were detected on the surface of the antennas, and these fields can be used to power sensors wirelessly. 


\section{Chapter 5}

\section{Rectangular Reconfigurable Antenna (RRA) with Ultra Wideband Tuning Ability}

\subsection{Introduction}

Reconfigurable antennas have been designed with the objective that an antenna should have multiple resonating frequencies, and should be able to modify its radiation pattern at will. Multiple designs of reconfigurable antennas [1] [2] have never been able to reveal the electric field equations for the antenna. Each iteration of a reconfigurable antenna will have a different resonating frequency and a different radiation pattern. An antenna has to be designed with the objective that it should exhibit broadband behaviour, and has to be compact.

Reconfigurable antennas can change their radiation patterns, as well as use the same amount of current supply to reach out to a farther distance. This research will advance the existing designs of reconfigurable antennas, as well as design a new reconfigurable antenna to enhance the performance of wireless devices and enable wireless powering. Instead of increasing the input excitation current or voltage supply to the antenna, the number of radiating surfaces can be increased to form a dynamic reconfigurable antenna which can change its size or radiation pattern depending on what the application requires. A reconfigurable antenna can work best at more than three frequencies, if designed optimally. Reconfigurable antennas are cost effective to manufacture [1], very easy to fabricate, have higher bandwidth, and favourable electrical performance. Reconfigurable antennas have multiple applications such as Bluetooth, Satellite Digital Multimedia Broadcasting and Wireless Local Area Network (WLAN).

A Rectangular Reconfigurable Antenna (RRA) is presented in this chapter. The antenna system consists of 8 rectangular radiating patches. The power radiated equation, as well as the equivalent circuit for the antenna is presented in this chapter. The electric field equations for 
this antenna are also presented. A detailed investigation of this antenna, as well as the simulation results showing the s- parameters and the radiation characteristics, are presented and discussed. Finally, experimental results from the network analyser are also presented.

\subsection{RRA}

The Rectangular Reconfigurable Antenna (RRA) [4] shown in fig. 5.1 consists of nine radiating elements and eight switches. Simulations were conducted with an FR4 epoxy, as well as an RT Durroid 5880 substrate and a ground plane. The first rectangle connects to the first ring of rectangles through four switches, and the first ring of rectangles connects to the second

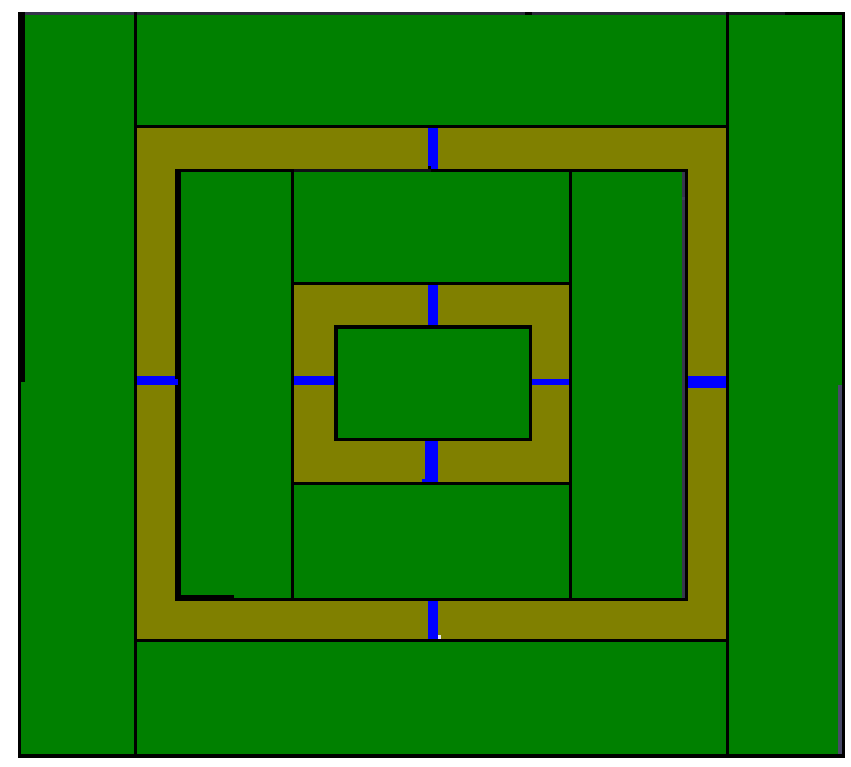

Fig. 5.1 RRA

ring of rectangles through four other switches. The Rectangular Reconfigurable Antenna [4] consists of three iterations. When the first rectangle is radiating, it is considered to be the first iteration; when the first set of four switches is on, it is considered to be the second iteration; and when the second set of four switches is on, it is considered to be the third iteration.

\subsection{Design of the RRA}

In order to understand how the RRA was designed, it is necessary to understand a PCB and the design of a basic rectangular patch antenna. The design of the RRA and its equivalent circuit is also presented in this section. 


\subsubsection{Printed Circuit Boards}

An antenna is made out of a double-sided Printed Circuit Board (PCB). Double-sided PCBs as shown in fig. 5.2, contain metal on the top and at the bottom. The middle layer is a substrate such as the FR4 epoxy or the RT Durroid 5880. Once the design of the antenna is decided on, the top metal layer of the PCB that has to be removed is taken off by a process called etching. A drilling machine such as the S65 Potomac can also be used to remove the metal layer. Generally, copper is used for the top and the bottom layers. A capacitor is made of two metals with a substrate in between. Since the PCBs replicate a capacitor, each PCB board comes with the specifications of capacitance losses, thickness of the copper sheets and the substrate.

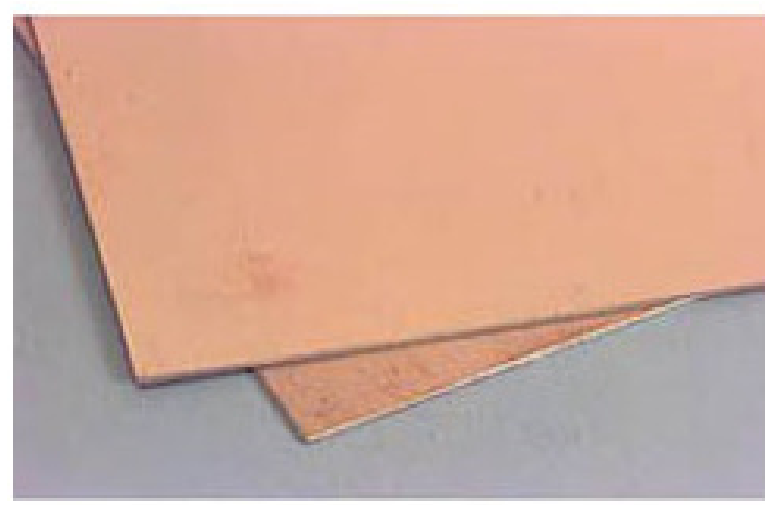

Fig. 5.2 PCB

\subsubsection{Antenna Design}

As shown in fig. 5.3, an antenna is designed on the bases of its width and the height from the ground plane to the patch.

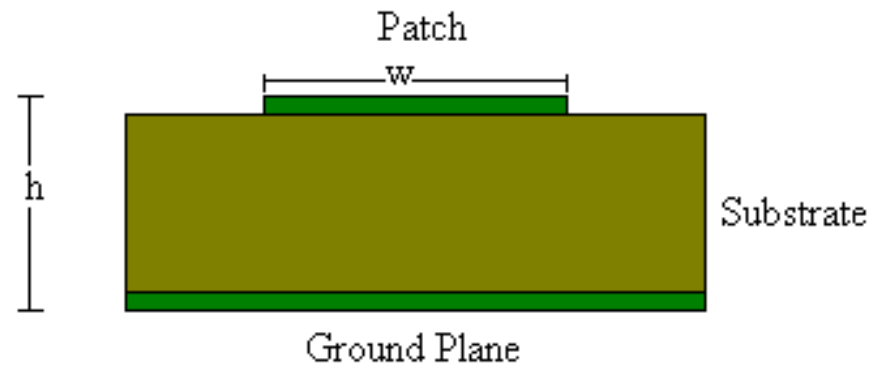

Fig. 5.3 A Typical Antenna Design 
The following formula is used to find out whether an antenna would work in a frequency range (GHz or MHz)

$$
\lambda=\frac{\mathrm{c}}{\mathrm{f}}
$$

where $\mathrm{c}$ is the velocity of light, $\mathrm{f}$ is the frequency and $\lambda$ is the wavelength.

So if we want to find out if an antenna would work in a frequency range, then let us assume the maximum frequency is $15 \mathrm{GHz}$. From the above formula, we have

$$
\begin{gathered}
\lambda=\frac{3 \times 10^{10}}{15 \times 10^{9}} \\
\lambda=2 \mathrm{~cm}=20 \mathrm{~mm}
\end{gathered}
$$

So now we know that if the maximum dimension (from one end of the antenna to the other end) exceeds $20 \mathrm{~mm}$, then the antenna would not work. The maximum dimension of the antenna could be stretched to about 25 or $28 \mathrm{~mm}$.

\subsubsection{Cavity Model}

Microstrip antennas [13] behave like dielectric load cavities and exhibit higher order resonances. The fields between the patch and the ground plane can be found out by treating the region within the dielectric substrate as a cavity bounded by electric conductors (above and below it), and by magnetic walls. A rectangular patch antenna has four Transverse Modes, $\mathrm{TM}^{\mathrm{x}}{ }_{010}, \mathrm{TM}^{\mathrm{x}}{ }_{001}, \mathrm{TM}^{\mathrm{x}}{ }_{020}, \mathrm{TM}^{\mathrm{x}}{ }_{002}$. In the $\mathrm{TM}$ modes (Transverse Magnetic), there is no magnetic field in the direction of propagation.

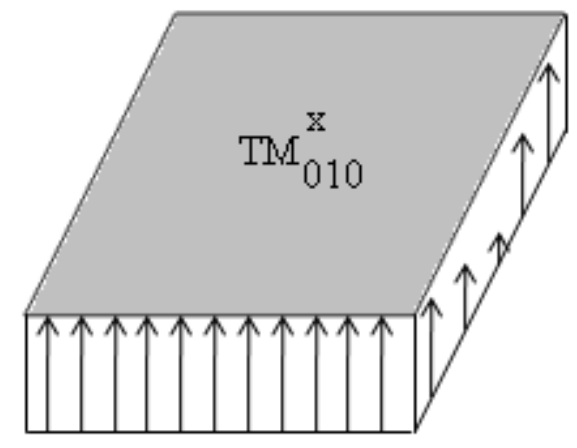

Fig. 5.4 $\mathrm{TM}^{\mathrm{x}}{ }_{010}$ of a rectangular patch antenna 


\subsection{Field Configurations (Modes) -TM ${ }^{x}$}

The vector potential $A_{x}$ satisfies the homogeneous wave equation of

$$
\nabla^{2} A_{x}+k^{2} A_{x}=0
$$

$A_{x}$ is given by

$$
A_{x}=\left[A_{1} \cos \left(k_{x} x\right)+B_{1} \sin \left(k_{x} x\right)\right]\left[A_{2} \cos \left(k_{y} y\right)+B_{2} \sin \left(k_{y} y\right)\right]\left[A_{3} \cos \left(k_{z} z\right)+B_{3} \sin \left(k_{z} z\right)\right]
$$

$\mathrm{k}_{\mathrm{x}}, \mathrm{k}_{\mathrm{y}}, \mathrm{k}_{\mathrm{z}}$ are wavenumbers along the $\mathrm{x}, \mathrm{y}$ and $\mathrm{z}$ directions.

The electric and magnetic fields within the cavity are related to the vector potential $A_{x}$ by

$$
\begin{array}{lc}
\mathrm{E}_{\mathrm{x}}=-\mathrm{j} \frac{1}{\omega \mu \varepsilon}\left(\frac{\partial^{2}}{\partial \mathrm{x}^{2}}+\mathrm{k}^{2}\right) \mathrm{A}_{\mathrm{x}} & \mathrm{H}_{\mathrm{x}}=0 \\
\mathrm{E}_{\mathrm{y}}=-\mathrm{j} \frac{1}{\omega \mu \varepsilon} \frac{\partial^{2} \mathrm{~A}_{\mathrm{x}}}{\partial \mathrm{x} \partial \mathrm{y}} & \mathrm{H}_{\mathrm{y}}=\frac{1}{\mu} \frac{\partial \mathrm{A}_{\mathrm{x}}}{\partial \mathrm{z}} \\
\mathrm{E}_{\mathrm{z}}=-\mathrm{j} \frac{1}{\omega \mu \varepsilon} \frac{\partial^{2} \mathrm{~A}_{\mathrm{x}}}{\partial \mathrm{x} \partial \mathrm{z}} & \mathrm{H}_{\mathrm{z}}=\frac{1}{\mu} \frac{\partial \mathrm{A}_{\mathrm{x}}}{\partial \mathrm{y}}
\end{array}
$$

subject to the boundary conditions of

$$
\begin{aligned}
& \mathrm{E}_{\mathrm{y}}\left(\mathrm{x}^{\mid}=0,0 \leq \mathrm{y}^{\prime} \leq \mathrm{L}, 0 \leq \mathrm{z}^{\prime} \leq \mathrm{W}\right)=\mathrm{E}_{\mathrm{y}}\left(\mathrm{x}^{\prime}=\mathrm{h}, 0 \leq \mathrm{y}^{\mid} \leq \mathrm{L}, 0 \leq \mathrm{z}^{\prime} \leq \mathrm{W}\right)=0 \\
& \mathrm{H}_{\mathrm{y}}\left(0 \leq \mathrm{x}^{\mid} \leq \mathrm{h}, 0 \leq \mathrm{y}^{\prime} \leq \mathrm{L}, \mathrm{z}^{\mid}=0\right)=\mathrm{H}_{\mathrm{y}}\left(0 \leq \mathrm{x}^{\mid} \leq \mathrm{h}, 0 \leq \mathrm{y}^{\prime} \leq \mathrm{L}, \mathrm{z}^{\prime}=W\right)=0 \\
& \mathrm{H}_{\mathrm{z}}\left(0 \leq \mathrm{x}^{\mid} \leq \mathrm{h}, \mathrm{y}^{\mid}=0,0 \leq \mathrm{z}^{\prime} \leq \mathrm{W}\right)=\mathrm{H}_{\mathrm{y}}\left(0 \leq \mathrm{x}^{\mid} \leq \mathrm{h}, \mathrm{y}^{\mid}=\mathrm{L}, 0 \leq \mathrm{z}^{\mid} \leq \mathrm{W}\right)=0
\end{aligned}
$$

The primed coordinates $\mathrm{x}^{\prime}, \mathrm{y}^{\prime}, \mathrm{z}^{\prime}$ are used to represent the fields within the cavity. When the above boundary conditions are applied, it can be shown that the wavenumbers $\mathrm{k}_{\mathrm{x}}, \mathrm{k}_{\mathrm{y}}, \mathrm{k}_{\mathrm{z}}$ are 
given by

$$
\left.\begin{array}{l}
\mathrm{k}_{\mathrm{x}}=\left(\frac{\mathrm{m} \pi}{\mathrm{h}}\right), \mathrm{m}=0,1,2, \ldots . . \\
\mathrm{k}_{\mathrm{y}}=\left(\frac{\mathrm{n} \pi}{\mathrm{L}}\right), \mathrm{n}=0,1,2, \ldots . . \\
\mathrm{k}_{\mathrm{z}}=\left(\frac{\mathrm{p} \pi}{\mathrm{W}}\right), \mathrm{p}=0,1,2, \ldots . .
\end{array}\right\} \mathrm{m}=\mathrm{n}=\mathrm{p} \neq 0
$$

The vector potential $A_{x}$ within the cavity is

$$
A_{x}=A_{m n p} \cos \left(k_{x} x^{\prime}\right) \cos \left(k_{y} y^{\prime}\right) \cos \left(k_{z} z^{\prime}\right)
$$

Where $A_{m n p}$ represents the amplitude coefficient of each mnp mode.

Hence, the wavenumbers $\mathrm{k}_{\mathrm{x}}, \mathrm{k}_{\mathrm{y}}, \mathrm{k}_{\mathrm{z}}$ are subject to the constraint equation

$$
\mathrm{k}_{\mathrm{x}}^{2}+\mathrm{k}_{\mathrm{y}}^{2}+\mathrm{k}_{\mathrm{z}}^{2}=\left(\frac{\mathrm{m} \pi}{\mathrm{h}}\right)^{2}+\left(\frac{\mathrm{n} \pi}{\mathrm{L}}\right)^{2}+\left(\frac{\mathrm{p} \pi}{\mathrm{W}}\right)^{2}=\mathrm{k}_{\mathrm{r}}{ }^{2}=\omega_{\mathrm{r}}{ }^{2} \mu \varepsilon
$$

The resonant frequencies of the cavity are given by

$$
\left(f_{r}\right)_{m n p}=\frac{1}{2 \pi \sqrt{\mu \varepsilon}} \sqrt{\left(\frac{m \pi}{h}\right)^{2}+\left(\frac{n \pi}{L}\right)^{2}+\left(\frac{p \pi}{W}\right)^{2}}
$$

The electric and magnetic fields within the cavity, because of a rectangular patch antenna are given by

$$
\begin{gathered}
E_{x}=-j \frac{\left(k^{2}-k_{x}^{2}\right)}{\omega \mu \varepsilon} A_{m n p} \cos \left(k_{x} x^{\prime}\right) \cos \left(k_{y} y^{\prime}\right) \cos \left(k_{z} z^{\prime}\right) \\
E_{y}=-j \frac{k_{x} k_{y}}{\omega \mu \varepsilon} A_{m n p} \sin \left(k_{x} x^{\prime}\right) \sin \left(k_{y} y^{\prime}\right) \cos \left(k_{z} z^{\prime}\right) \\
E_{z}=-j \frac{k_{x} k_{z}}{\omega \mu \varepsilon} A_{m n p} \sin \left(k_{x} x^{\prime}\right) \cos \left(k_{y} y^{\prime}\right) \sin \left(k_{z} z^{\prime}\right) \\
H_{x}=0
\end{gathered}
$$




$$
\begin{gathered}
\mathrm{H}_{\mathrm{y}}=-\mathrm{j} \frac{\mathrm{k}_{\mathrm{z}}}{\mu} \mathrm{A}_{\mathrm{mnp}} \cos \left(\mathrm{k}_{\mathrm{x}} \mathrm{x}^{\prime}\right) \cos \left(\mathrm{k}_{\mathrm{y}} \mathrm{y}^{\prime}\right) \sin \left(\mathrm{k}_{\mathrm{z}} \mathrm{z}^{\prime}\right) \\
\mathrm{H}_{\mathrm{z}}=\frac{\mathrm{k}_{\mathrm{y}}}{\mu} \mathrm{A}_{\mathrm{mnp}} \cos \left(\mathrm{k}_{\mathrm{x}} \mathrm{x}^{\prime}\right) \sin \left(\mathrm{k}_{\mathrm{y}} \mathrm{y}^{\prime}\right) \cos \left(\mathrm{k}_{\mathrm{z}} \mathrm{z}^{\prime}\right)
\end{gathered}
$$

The electric and magnetic fields within the cavity of the RRA are given by

$$
\begin{gathered}
\mathrm{E}_{\mathrm{x}}=-\mathrm{j} \frac{\mathrm{n}\left(\mathrm{k}^{2}-\mathrm{k}_{\mathrm{x}}^{2}\right)}{\omega \mu \varepsilon} \mathrm{A}_{\mathrm{mnp}} \cos \left(\mathrm{k}_{\mathrm{x}} \mathrm{x}^{\prime}\right) \cos \left(\mathrm{k}_{\mathrm{y}} \mathrm{y}^{\prime}\right) \cos \left(\mathrm{k}_{\mathrm{z}} \mathrm{z}^{\prime}\right) \\
\mathrm{E}_{\mathrm{y}}=-\mathrm{j} \frac{\mathrm{n} \mathrm{k}_{\mathrm{x}} \mathrm{k}_{\mathrm{y}}}{\omega \mu \varepsilon} \mathrm{A}_{\mathrm{mnp}} \sin \left(\mathrm{k}_{\mathrm{x}} \mathrm{x}^{\prime}\right) \sin \left(\mathrm{k}_{\mathrm{y}} \mathrm{y}^{\prime}\right) \cos \left(\mathrm{k}_{\mathrm{z}} \mathrm{z}^{\prime}\right) \\
\mathrm{E}_{\mathrm{z}}=-\mathrm{j} \frac{\mathrm{n} \mathrm{k}_{\mathrm{x}} \mathrm{k}_{\mathrm{z}}}{\omega \mu \varepsilon} \mathrm{A}_{\mathrm{mnp}} \sin \left(\mathrm{k}_{\mathrm{x}} \mathrm{x}^{\prime}\right) \cos \left(\mathrm{k}_{\mathrm{y}} \mathrm{y}^{\prime}\right) \sin \left(\mathrm{k}_{\mathrm{z}} \mathrm{z}^{\mid}\right) \\
\mathrm{H}_{\mathrm{y}}=-\mathrm{j} \frac{\mathrm{n} \mathrm{k}_{\mathrm{z}}}{\mu} \mathrm{A}_{\mathrm{mnp}} \cos \left(\mathrm{k}_{\mathrm{x}} \mathrm{x}^{\prime}\right) \cos \left(\mathrm{k}_{\mathrm{y}} \mathrm{y}^{\prime}\right) \sin \left(\mathrm{k}_{\mathrm{z}} \mathrm{z}^{\prime}\right) \\
\mathrm{H}_{\mathrm{z}}=\frac{\mathrm{nk} \mathrm{k}_{\mathrm{y}}}{\mu} \mathrm{A}_{\mathrm{mnp}} \cos \left(\mathrm{k}_{\mathrm{x}} \mathrm{x}^{\prime}\right) \sin \left(\mathrm{k}_{\mathrm{y}} \mathrm{y}^{\prime}\right) \cos \left(\mathrm{k}_{\mathrm{z}} \mathrm{z}^{\prime}\right)
\end{gathered}
$$

Where $\mathrm{n}$ denotes the number of rectangles that are radiating, and its range is given by $0<n<$ 9.

\subsection{Fields Radiated (Radiating Slots) $-T M_{010}{ }^{x}$}

The far-zone electric fields radiated by each rectangular patch can be written as

$$
\begin{gathered}
\mathrm{E}_{\mathrm{r}} \simeq \mathrm{E}_{\theta} \simeq 0 \\
\mathrm{E}_{\varnothing}=+\mathrm{j} \frac{\mathrm{k}_{0} \mathrm{hWE}_{0} \mathrm{e}^{-\mathrm{jk_{0 }} \mathrm{r}}}{2 \pi \mathrm{r}}\left\{\sin \theta \frac{\sin (\mathrm{X})}{\mathrm{X}} \frac{\sin (\mathrm{Z})}{\mathrm{Z}}\right\}
\end{gathered}
$$


Where

$$
\begin{gathered}
\mathrm{X}=\frac{\mathrm{k}_{0} \mathrm{~h}}{2} \sin \theta \cos \emptyset \\
\mathrm{Z}=\frac{\mathrm{k}_{0} \mathrm{~W}}{2} \cos \theta
\end{gathered}
$$

For very small heights, $\mathrm{E}_{\varnothing}$ reduces to

$$
E_{\varnothing}=+j \frac{V_{0} e^{-j k_{0} r}}{\pi r}\left\{\sin \theta \frac{\sin \left(\frac{k_{0} W}{2} \cos \theta\right)}{\cos \theta}\right\}
$$

Where $\mathrm{V}_{0}=\mathrm{hE}_{0}$

\subsubsection{Directivity}

Directivity of an antenna is given by the formula

$$
\mathrm{D}_{0}=\frac{\mathrm{U}_{\max }}{\mathrm{U}_{0}}=\frac{4 \pi \mathrm{U}}{\mathrm{P}_{\mathrm{rad}}}
$$

Using the electric field equation for very small heights in section 5.3.2.1.2, the maximum radiation intensity and radiated power for a rectangular patch antenna can be written as

$$
\begin{gathered}
U_{\text {max }}=\frac{\left|V_{0}\right|^{2}}{2 \eta_{0} \pi^{2}}\left(\frac{\pi W}{\lambda_{0}}\right)^{2} \\
P_{\text {rad }}=\frac{\left|V_{0}\right|^{2}}{2 \pi \eta_{0}} \int_{0}^{\pi}\left[\frac{\sin \left(\frac{\mathrm{k}_{0} W}{2} \cos \theta\right)}{\cos \theta}\right]^{2} \sin ^{3} \theta
\end{gathered}
$$

Thus, the radiated power of the RRA can be written as

$$
P_{\text {rad }}=\frac{\left|V_{0}\right|^{2}}{2 \pi \eta_{0}} \int_{0}^{\pi}\left[\frac{\sin \left(\frac{\mathrm{k}_{0}\left(\sum_{\mathrm{n}=1}^{9} \mathrm{~W}_{\mathrm{n}}\right)}{2} \cos \theta\right)}{\cos \theta}\right]^{2} \sin ^{3} \theta
$$


Where $\mathrm{W}$ denotes the width of the rectangular patch and $\mathrm{n}$ denotes the number of rectangles that are radiating.

The directivity of a single patch is

$$
\mathrm{D}_{0}=\left(\frac{2 \pi \mathrm{W}}{\lambda_{0}}\right)^{2} \frac{1}{\mathrm{I}_{1}}
$$

Thus, the directivity of the RRA is

$$
\mathrm{D}_{0}=\left(\frac{2 \pi\left(\sum_{\mathrm{n}=1}^{9} \mathrm{~W}_{\mathrm{n}}\right)}{\lambda_{0}}\right)^{2} \frac{1}{\mathrm{I}_{1}}
$$

Where $\mathrm{I}_{1}$ for a single slot is given by

$$
\begin{gathered}
I_{1}=\int_{0}^{\pi[}\left[\frac{\sin \left(\frac{\mathrm{k}_{0} \mathrm{~W}}{2} \cos \theta\right)}{\cos \theta}\right]^{2} \sin ^{3} \theta \\
=-2+\cos (\mathrm{X})+\mathrm{XS} \text { i }(\mathrm{X})+\frac{\sin (\mathrm{X})}{\mathrm{X}} \\
\mathrm{X}=\mathrm{k}_{0} \mathrm{~W}
\end{gathered}
$$

$\mathrm{I}_{1}$ for the RRA is given by

$$
I_{1}=\int_{0}^{\pi}\left[\frac{\sin \left(\frac{k_{0}\left(\sum_{n=1}^{9} W_{n}\right)}{2} \cos \theta\right)}{\cos \theta}\right]^{2} \sin ^{3} \theta
$$

\subsubsection{Transmission Line Model}

A microstrip antenna behaves like a homogeneous line of one dielectric [13] (only the substrate), and the effective dielectric constant of the antenna approaches the value of the dielectric constant of the substrate. For low frequencies, the effective dielectric of a rectangular patch antenna is constant. At intermediate frequencies, its values initially increase and 
approach the dielectric constant of the substrate. The effective dielectric constant of a rectangular patch antenna is given by

$$
\varepsilon_{\mathrm{reff}}=\frac{\varepsilon_{\mathrm{r}}+1}{2}+\frac{\varepsilon_{\mathrm{r}}-1}{2}\left[1+12 \frac{\mathrm{h}}{\mathrm{w}}\right]^{-1 / 2}
$$

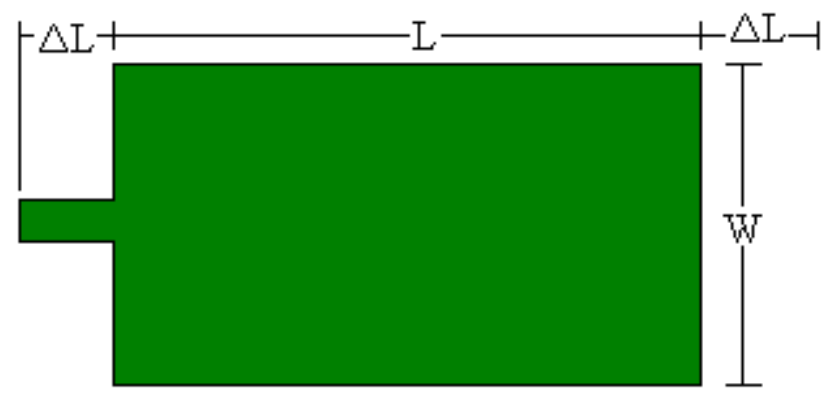

Fig. 5.5 Effective and physical lengths of a rectangular patch antenna [13]

The effective length of the patch antenna is given by

$$
\Delta \mathrm{L}=0.412 \mathrm{~h} \frac{\left(\varepsilon_{\text {reff }}+0.3\right)\left(\frac{\mathrm{W}}{\mathrm{h}}+0.264\right)}{\left(\varepsilon_{\text {reff }}-0.258\right)\left(\frac{\mathrm{W}}{\mathrm{h}}+0.8\right)}
$$

Since the length of the patch is extended by $\Delta \mathrm{L}$ on both sides, the effective length would thus become

$$
\mathrm{L}_{\mathrm{eff}}=\mathrm{L}+2 \Delta \mathrm{L}
$$

A rectangular patch antenna has all its electric field lines directed into the substrate. Thus the rectangular patch antenna does not have magnetic field in the direction of propagation (TM transverse mode). To determine the dominant mode with the lowest resonance, the resonant frequencies need to be examined. For all microstrip antennas $\mathrm{h} \ll\langle\mathrm{L}$ and $\mathrm{h} \gg \mathrm{W}$. For the dominant $\mathrm{TM}_{010}$ mode where $\mathrm{L}>\mathrm{W}>\mathrm{h}$ (as shown in fig. 5.4), the resonating frequency (Hz) of the rectangular patch antenna is a function of the length given by

$$
\left(f_{r}\right)_{010}=\frac{1}{2 \mathrm{~L} \sqrt{\varepsilon_{\mathrm{r}}} \sqrt{\mu_{\mathrm{o}} \varepsilon_{0}}}=\frac{\mathrm{v}_{0}}{2 \mathrm{~L} \sqrt{\varepsilon_{\mathrm{r}}}}
$$


Where $\mathrm{v}_{0}$ is the free space velocity of light.

For an efficient radiator, a practical width [66] that leads to a good radiation pattern is

$$
\mathrm{w}=\frac{1}{2 \mathrm{f}_{\mathrm{r}} \sqrt{\mu_{0} \varepsilon_{0}}} \sqrt{\frac{2}{\varepsilon_{\mathrm{r}}+1}}=\frac{\mathrm{v}_{0}}{2 \mathrm{f}_{\mathrm{r}}} \sqrt{\frac{2}{\varepsilon_{\mathrm{r}}+1}}
$$

where $v_{0}$ is the free-space velocity of light and $f_{r}$ is the resonating frequency.

The length of the patch L can be determined by

$$
\mathrm{L}=\frac{1}{2 \mathrm{f}_{\mathrm{r}} \sqrt{\varepsilon_{\mathrm{reff}}} \sqrt{\mu_{0} \varepsilon_{0}}}-2 \Delta \mathrm{L}
$$

Since the length and the width of a rectangular patch antenna are inversely proportional to the resonating frequency, if the length and the width are varied, then the resonating frequency would also change. This idea of varying the length and the width to obtain multiple resonating frequencies was used to create the RRA. The centre rectangular patch of the RRA can be designed for a frequency, and an incremental length can be used to create rings of rectangles. The RRA has five rectangles which have different lengths and widths, thus giving it five different resonating frequencies.

\subsection{Conductance}

Each radiating rectangular patch is represented by a parallel equivalent admittance $\mathrm{Y}$ (with conductance $\mathrm{G}$ and susceptance B), as shown in fig. 5.6.

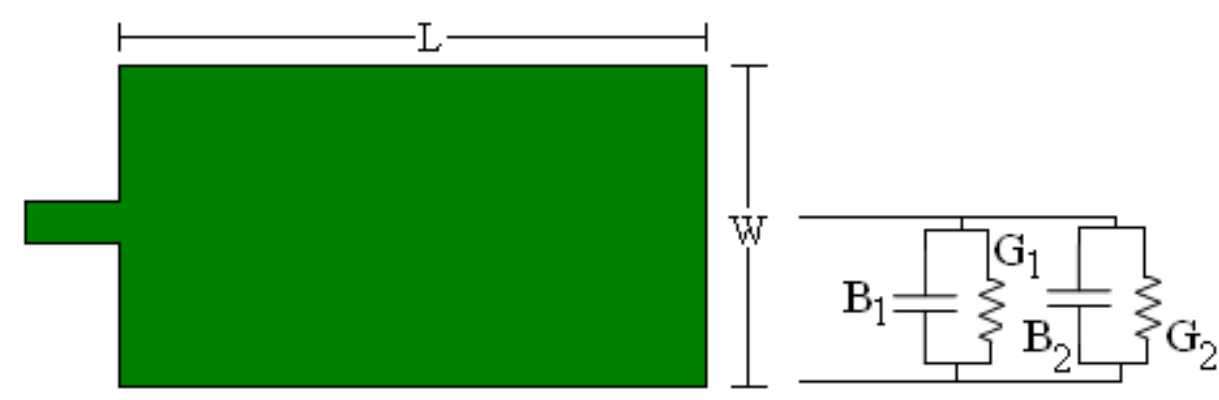

Fig. 5.6 Rectangular Patch with its equivalent circuit transmission model 
Since each radiating rectangular patch is represented by a parallel equivalent admittance, the RRA can be represented by a series of parallel equivalent admittances, as shown in fig. 5.7. The slots which represent the rectangular patches are numbered.

The equivalent admittance [13] of slot \# 1, shown in fig. 5.6, is given by

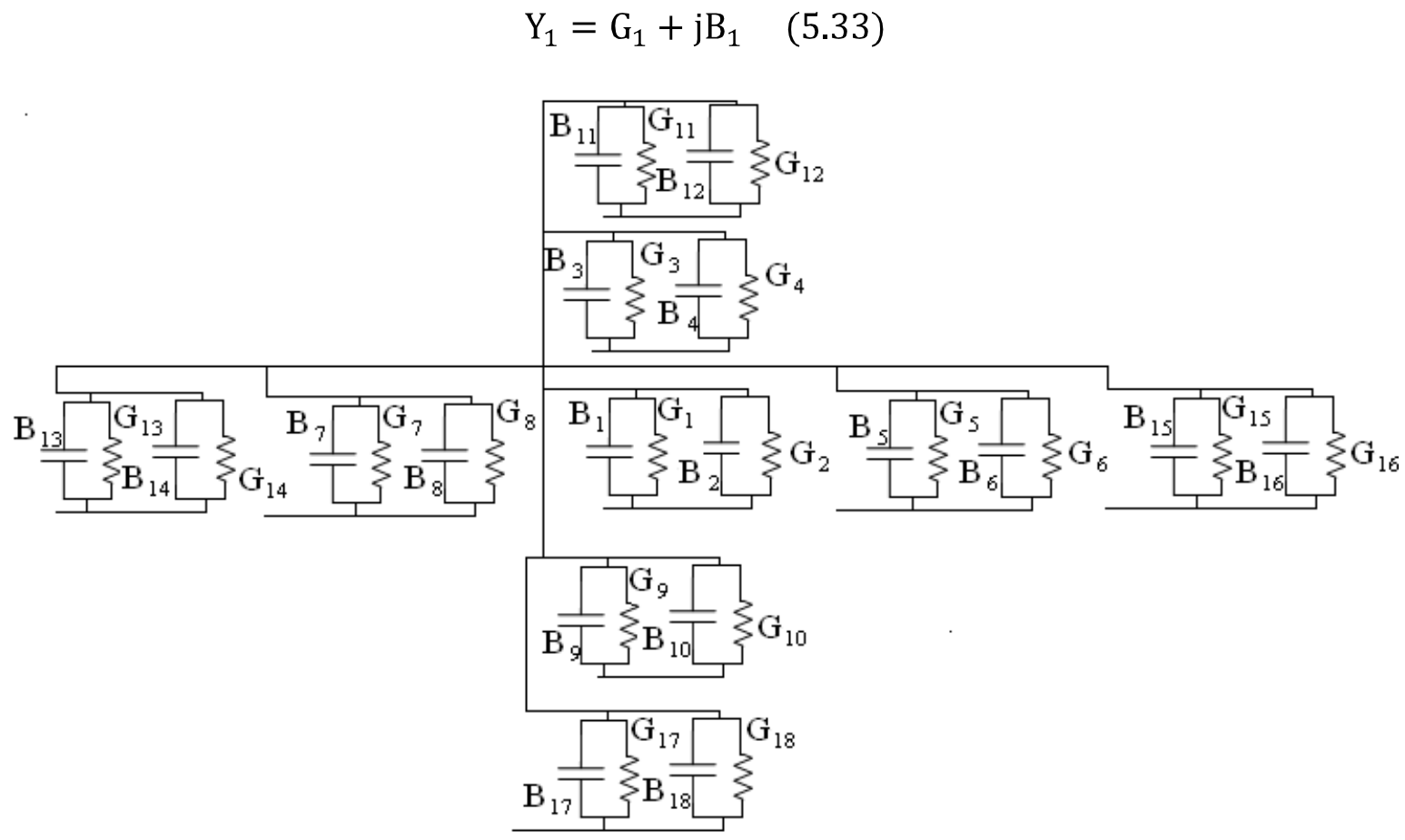

Fig. 5.7 Equivalent circuit transmission model of the RRA

For a slot of finite width $\mathrm{W}$

$$
\begin{aligned}
\mathrm{G}_{1}=\frac{\mathrm{W}}{120 \lambda_{0}}\left[1-\frac{1}{24}\left(\mathrm{k}_{0} \mathrm{~h}\right)^{2}\right] & \frac{\mathrm{h}}{\lambda_{0}}<\frac{1}{10}, \mathrm{k}_{0}=\omega_{0}{ }^{2} \mu \varepsilon \\
\mathrm{B}_{1}=\frac{\mathrm{W}}{120 \lambda_{0}}\left[1-0.636 \ln \left(\mathrm{k}_{0} \mathrm{~h}\right)\right] & \frac{\mathrm{h}}{\lambda_{0}}<\frac{1}{10}, \mathrm{k}=\omega_{0}{ }^{2} \mu \varepsilon
\end{aligned}
$$

Where $\mathrm{h}$ is the height of the antenna and $\lambda_{0}$ is the wavelength.

Since slot \# 2 is identical to slot \# 1, the equivalent admittances, conductances and susceptances are equal to each other. 
Thus,

$$
Y_{2}=Y_{1}, G_{2}=G_{1}, B_{2}=B_{1}
$$

The conductance of the first slot is given by

$$
\mathrm{G}_{1}=\frac{2 \mathrm{P}_{\mathrm{rad}}}{\left|\mathrm{V}_{0}\right|^{2}}
$$

Using the power radiated equation for a single rectangular patch, the conductance can be written as

$$
\mathrm{G}_{1}=\frac{\mathrm{I}_{1}}{120 \pi^{2}}
$$

Asymptotic values of the above two equations are

$$
G_{1}=\left\{\begin{array}{l}
\frac{1}{90}\left(\frac{W}{\lambda_{0}}\right)^{2} \mathrm{~W} \ll \lambda_{0} \\
\frac{1}{120}\left(\frac{\mathrm{W}}{\lambda_{0}}\right)^{2} \mathrm{~W} \gg \lambda_{0}
\end{array}\right.
$$

In order to derive the conductance and the susceptance of the RRA, the equivalent circuit and the design of the RRA have to be closely examined. The rings of rectangular radiators are attached to each other, as shown in fig. 5.1. The outer and inner rings of rectangular radiators are connected to each other through four switches, and the inner ring of radiators is connected to the central rectangular patch through four other switches.

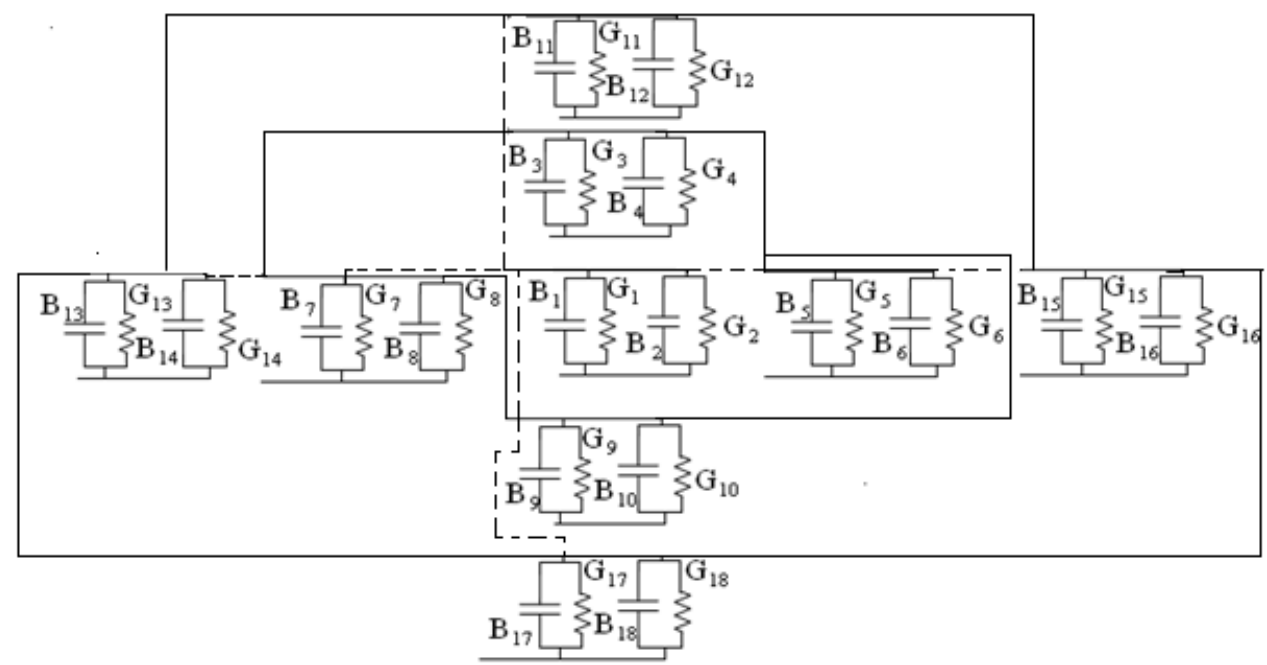

Fig. 5.8 Analysis of the Equivalent circuit transmission model of the RRA 
The connections between the equivalent circuits of the rectangles that are attached to each other are shown by solid lines, and the connections between the equivalent circuits of the rectangles that are connected to each other through switches are shown by dotted lines in fig. 5.8. The equivalent admittance of the RRA is a sum of all individual admittances and is given by

$$
\begin{array}{r}
Y_{1}=G_{1}+j B_{1} \\
Y_{e q}=\sum_{n=1}^{9} Y_{n}
\end{array}
$$

Thus the equivalent conductance and the equivalent susceptance are

$$
\begin{aligned}
& G_{e q}=\sum_{n=1}^{9} G_{n} \\
& B_{e q}=\sum_{n=1}^{9} B_{n}
\end{aligned}
$$

\subsection{Simulation of the RRA}

The Rectangular Reconfigurable antenna was excited with a coaxial probe and a slot, using an FR4 epoxy and an RT Durroid 5880 substrate, in Ansoft HFSS v11. The center rectangle was designed for a frequency of $10 \mathrm{GHz}$. This antenna was also implemented on a $\mathrm{PCB}$, and instead of switches, a copper surface of dimensions $2 \mathrm{~mm} \times 2 \mathrm{~mm}$ was used to connect the radiating parts in simulations, as well as in the PCB implementation. The center rectangle was first constructed with $2 \mathrm{~mm} \times 2 \mathrm{~mm}$ switches, and was surrounded by the first ring of rectangles. The second ring of rectangles was constructed in the same way. Initially, the antenna was designed for a maximum frequency of $10 \mathrm{GHz}$, but it was simulated and tested up to $15 \mathrm{GHz}$. Since the maximum frequency was thought to be $10 \mathrm{GHz}$, the maximum dimension 
can be calculated as

$$
\lambda=\frac{\mathrm{c}}{\mathrm{f}}
$$

Where $\mathrm{c}$ is the velocity of light and $\mathrm{f}$ is the maximum frequency. Substituting the values of $\mathrm{c}$ and $f$ we get

Finally we get

$$
\lambda=\frac{3 \times 10^{10}}{10^{10}}
$$

$$
\lambda=3 \mathrm{~cm} \text { or } 30 \mathrm{~mm}
$$

The maximum length of the substrate could not be more than $30 \mathrm{~mm}$, but in this case a substrate of length $33.06 \mathrm{~mm}$ and breadth $35.86 \mathrm{~mm}$ was used to simulate the antenna up to 15 $\mathrm{GHz}$ when it was excited using the coaxial probe feed. This decision to simulate and test the antenna up to $15 \mathrm{GHz}$ was taken, as there could be coupling between the radiating parts that could alter the results. These coupling effects could be reduced by introducing filters at the end points of the switches. The width of the centre rectangle is $\mathrm{w} 1=9.06 \mathrm{~mm}$ and the length is $11=11.86 \mathrm{~mm}$. The rectangles opposite to each other have the same dimensions in the first and the second rings of rectangles. The second ring of rectangles consists of four rectangles with dimensions: $12=13.06 \mathrm{~mm}$ and $\mathrm{w} 2=5 \mathrm{~mm} ; 13=5 \mathrm{~mm}$ and $\mathrm{w} 3=25.9 \mathrm{~mm}$. The dimensions of the third ring of rectangles are: $14=27.06 \mathrm{~mm}$ and $\mathrm{w} 4=3 \mathrm{~mm} ; 15=3 \mathrm{~mm}$ and $\mathrm{w} 5=35.89 \mathrm{~mm}$. The thickness of the radiating part and the ground plane is $0.017 \mathrm{~mm}$. This antenna would have five resonating frequencies in the third iteration, as this antenna has five dimensions of rectangles, and each particular dimension would produce one resonating frequency.

\subsubsection{Simulation of the RRA with a coaxial probe feed}

The radius of the inner cylinder of the coaxial probe was $1 \mathrm{~mm}$, and the height was taken to be $4 \mathrm{~mm}$. The input return losses, VSWR and the radiation pattern, were compared when the antenna was simulated using an FR4 epoxy and an RT Durroid 5880 substrate. 


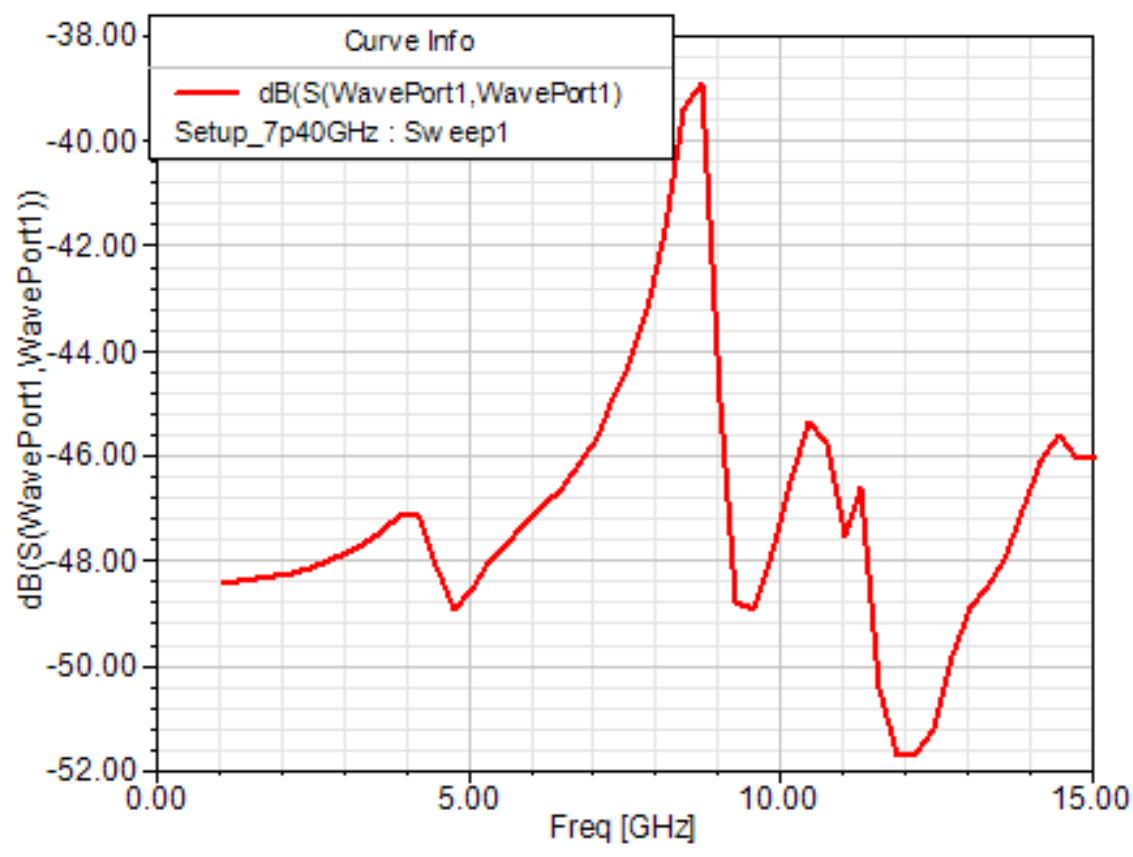

Fig. 5.9 Input Return Losses when the antenna was simulated using an RT Durroid 5880 substrate for the third iteration

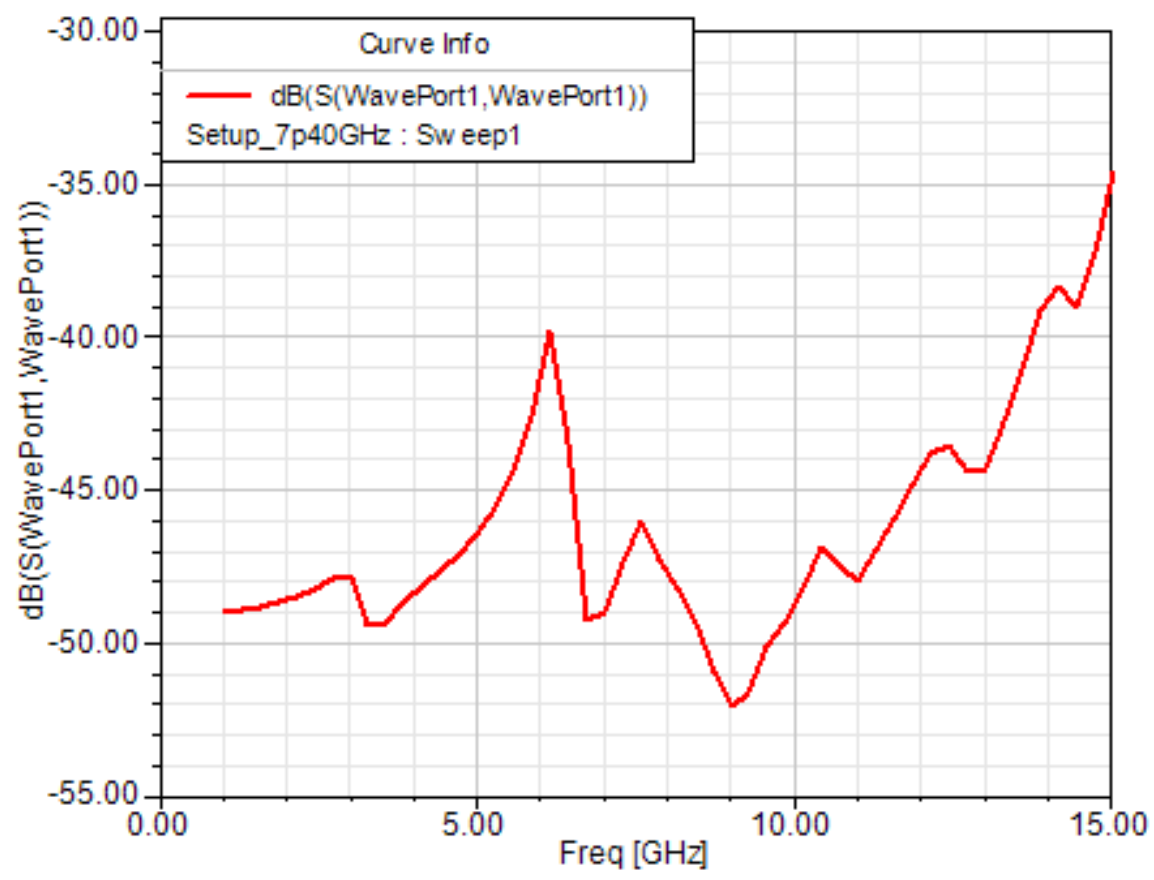

Fig. 5.10 Input Return Losses when the antenna was simulated using an FR4 epoxy substrate for the third iteration 


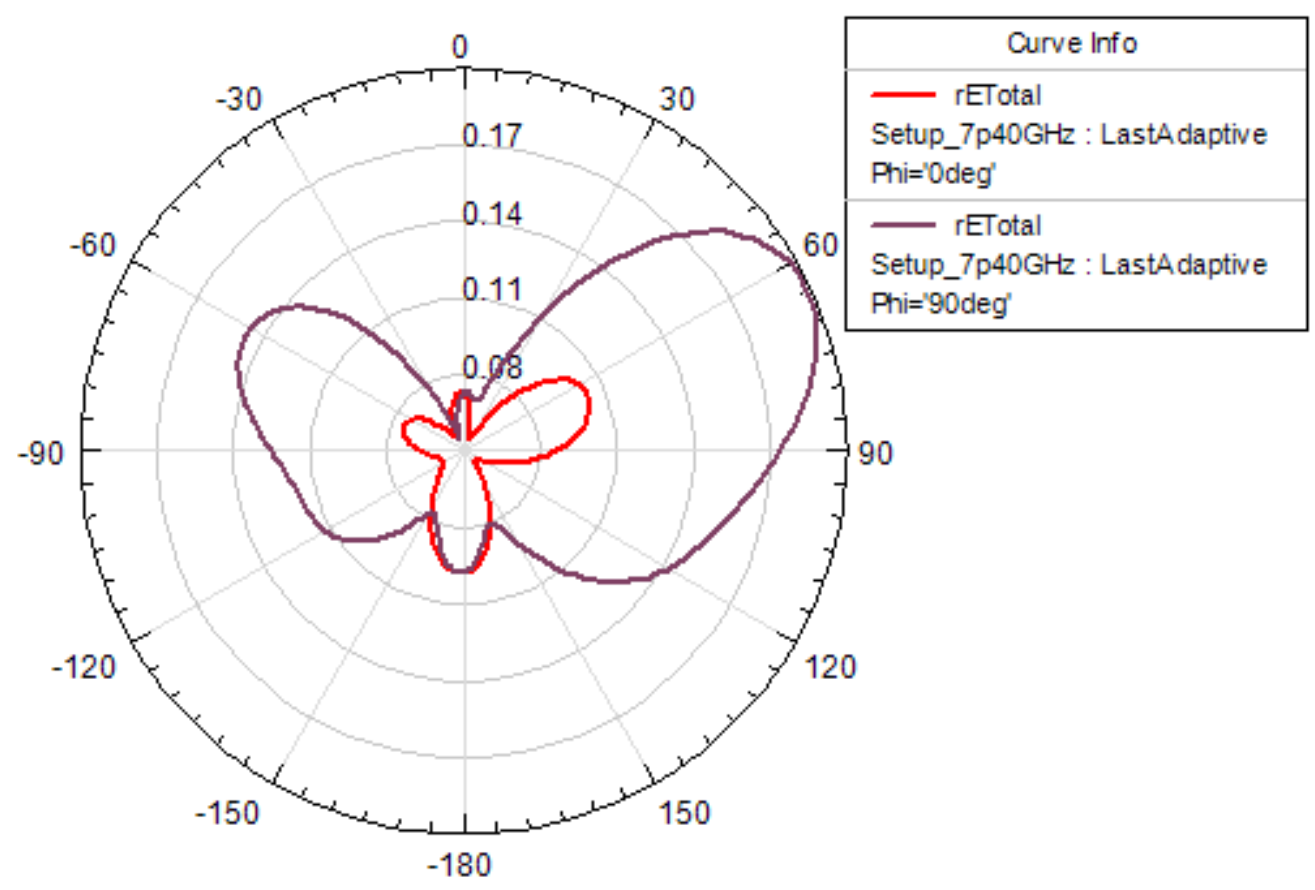

Fig. 5.11 Radiation Pattern when the antenna was simulated using an RT Durroid 5880 substrate for the third iteration

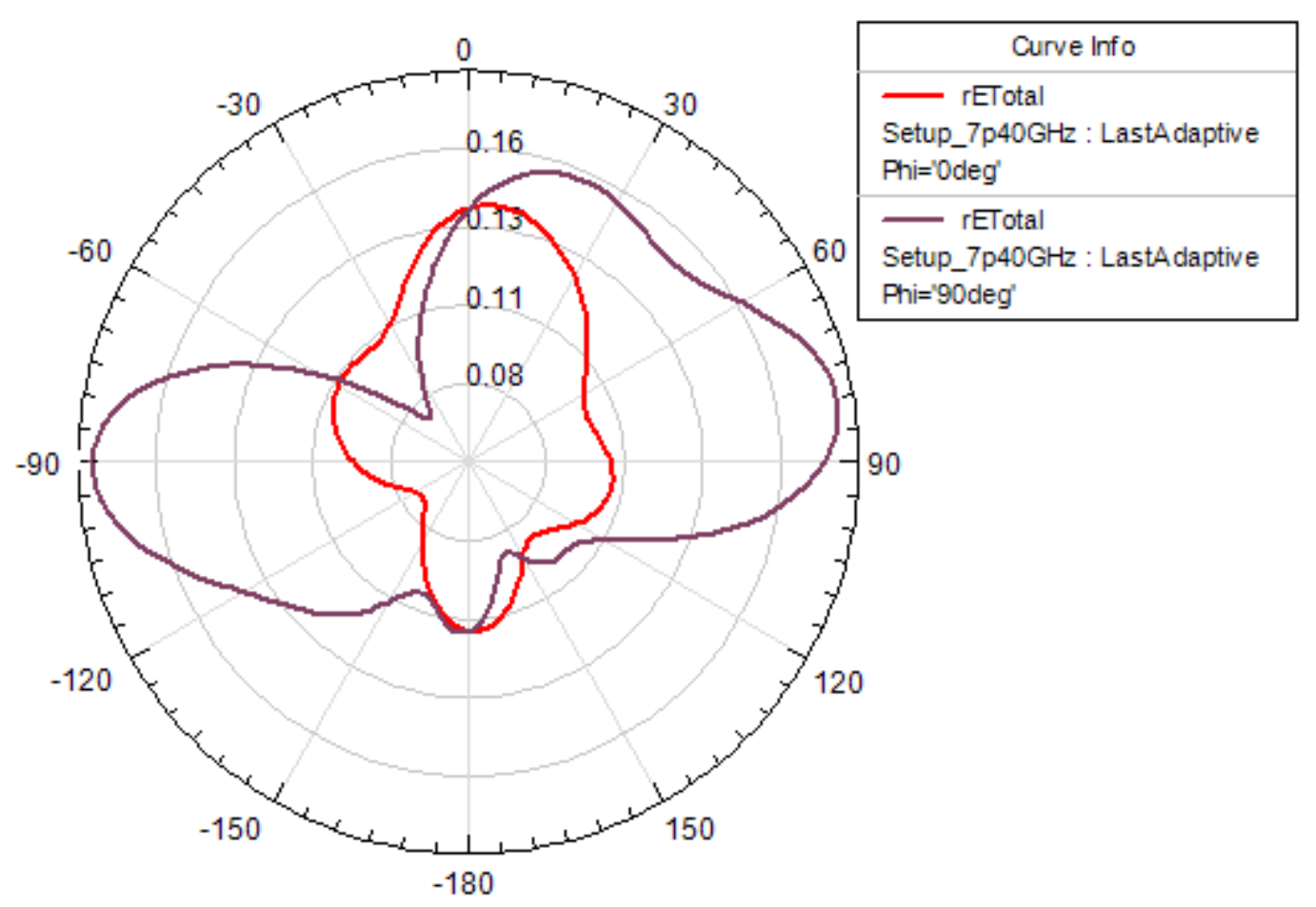

Fig. 5.12 Radiation Pattern when the antenna was simulated using an FR4 epoxy substrate for the third iteration 


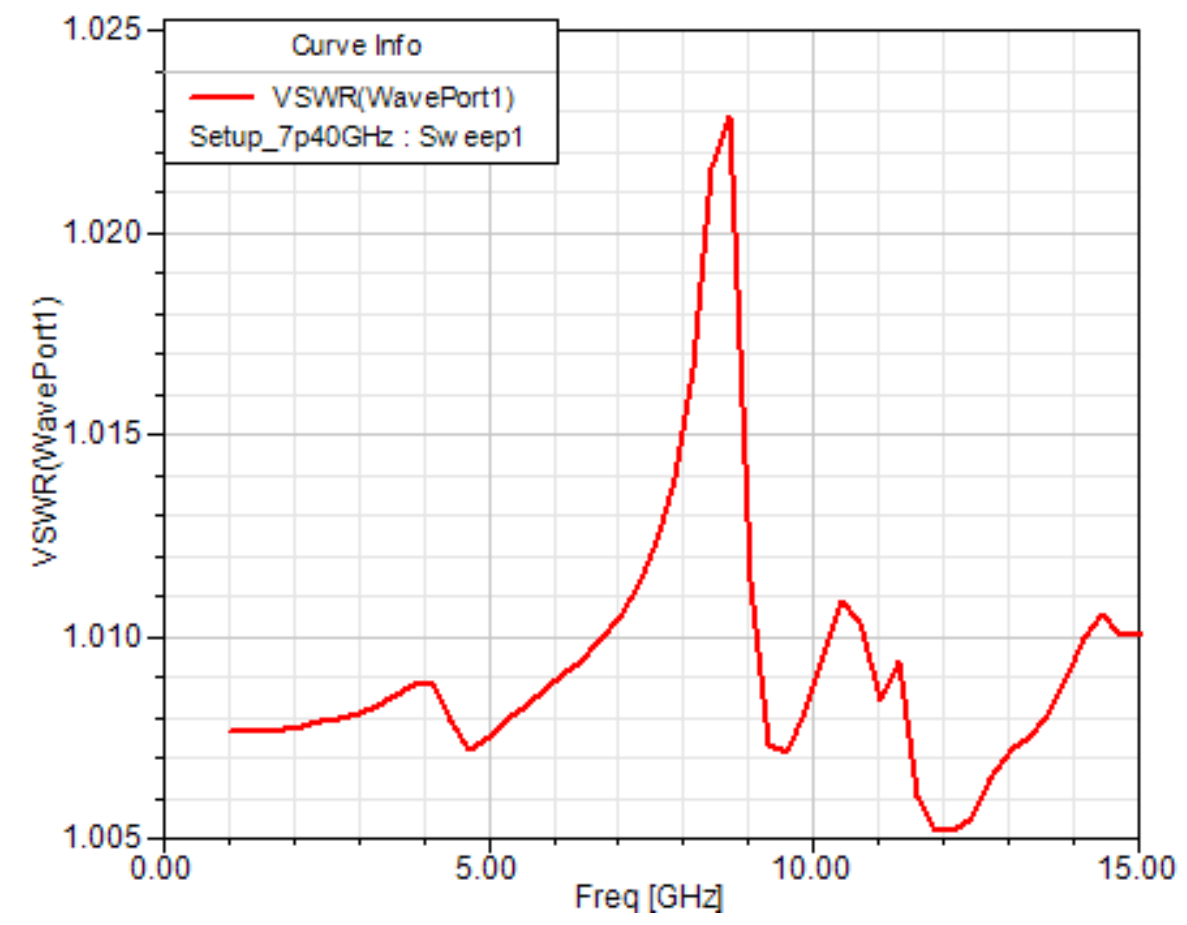

Fig. 5.13 VSWR when the antenna was simulated using an RT Durroid 5880 substrate for the third iteration

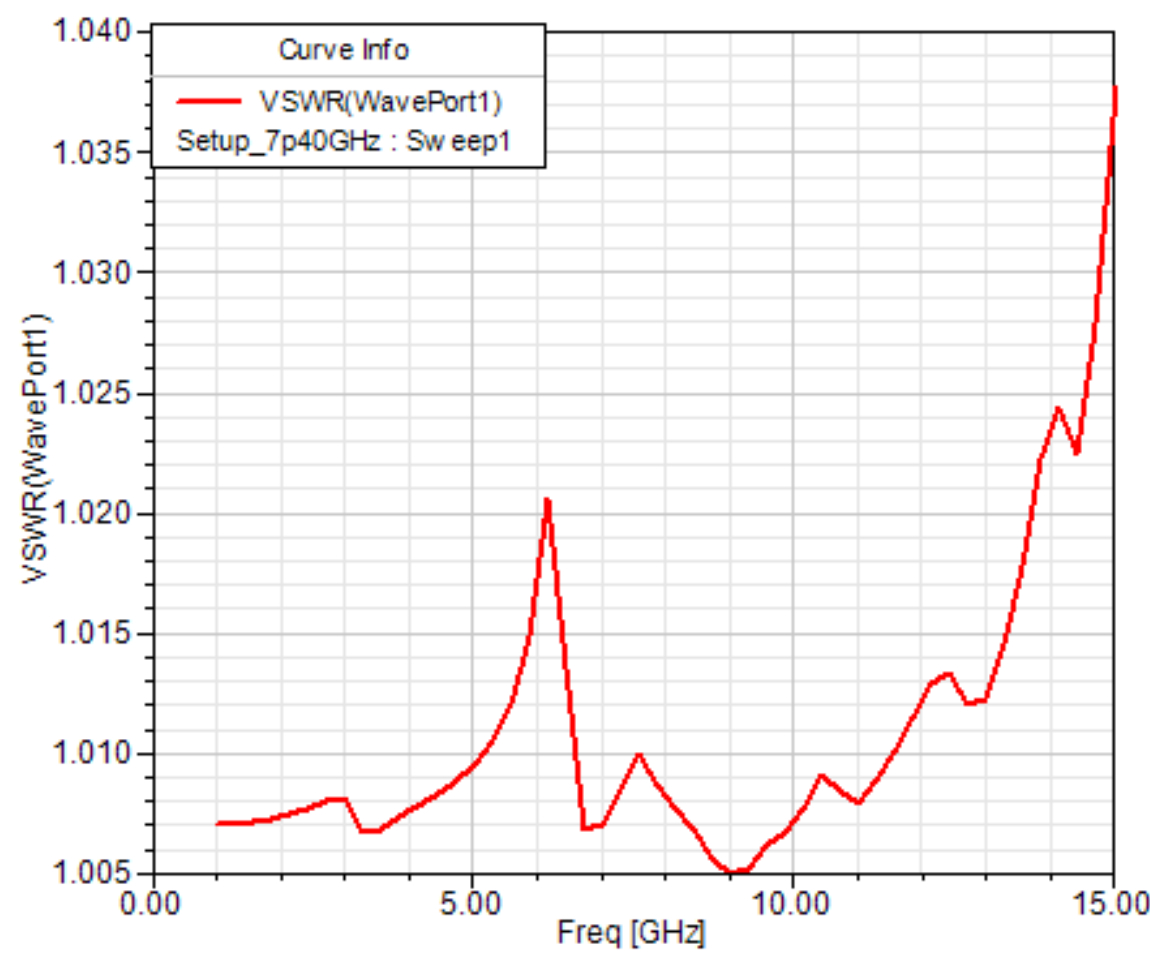

Fig. 5.14 VSWR when the antenna was simulated using an FR4 epoxy substrate for the third iteration 


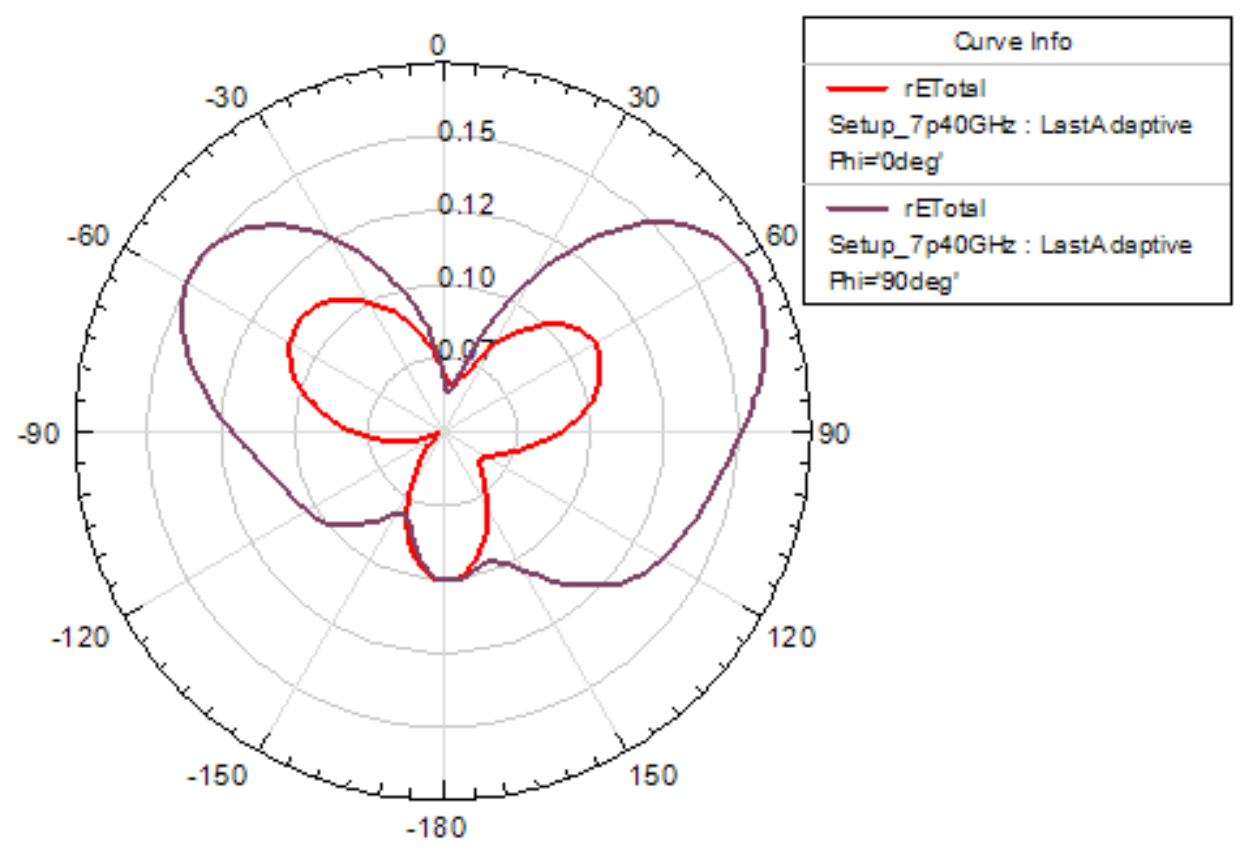

Fig. 5.15 Radiation Pattern when the antenna was simulated using an RT Durroid 5880 substrate for the second iteration

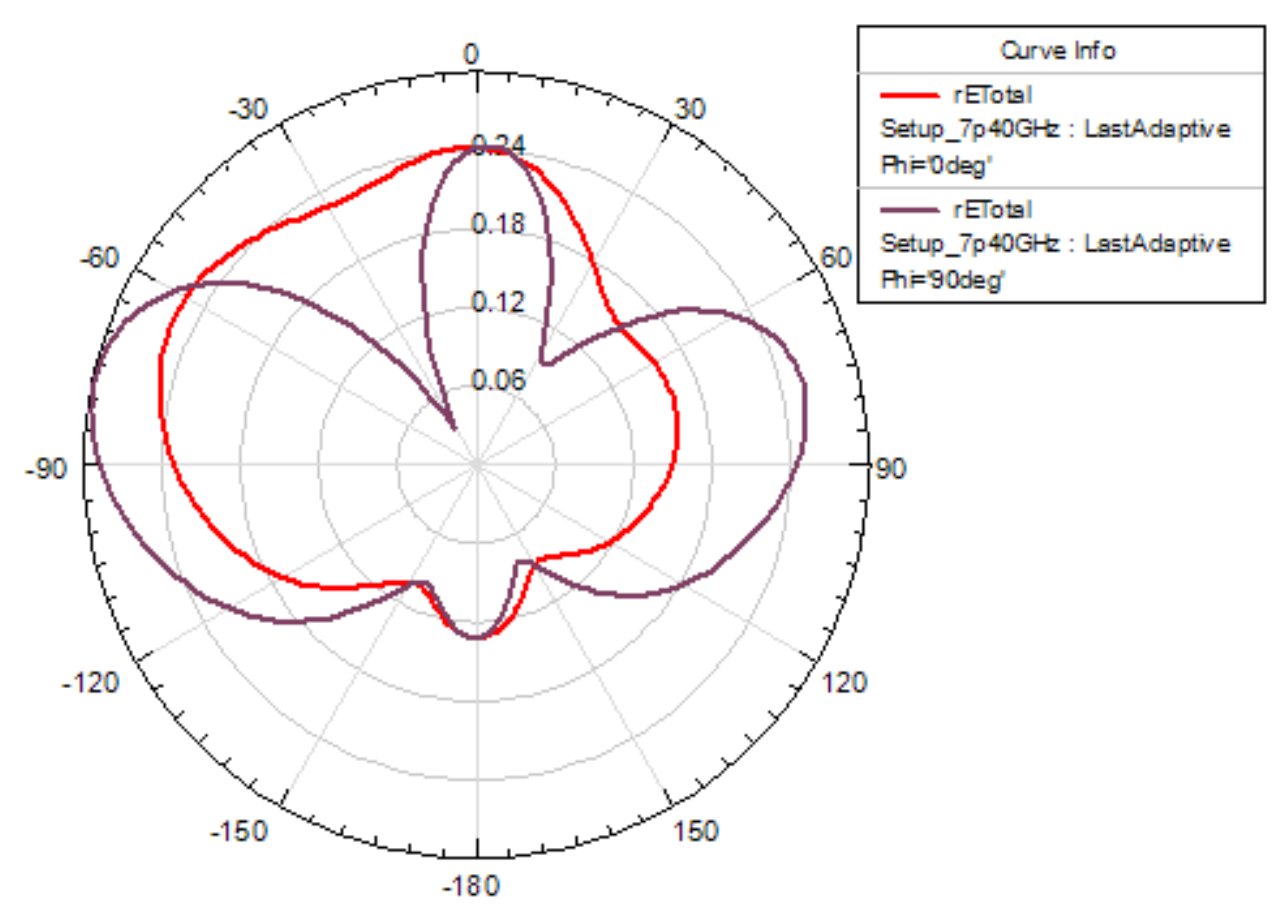

Fig. 5.16 Radiation Pattern when the antenna was simulated using an FR4 epoxy substrate for the second iteration 


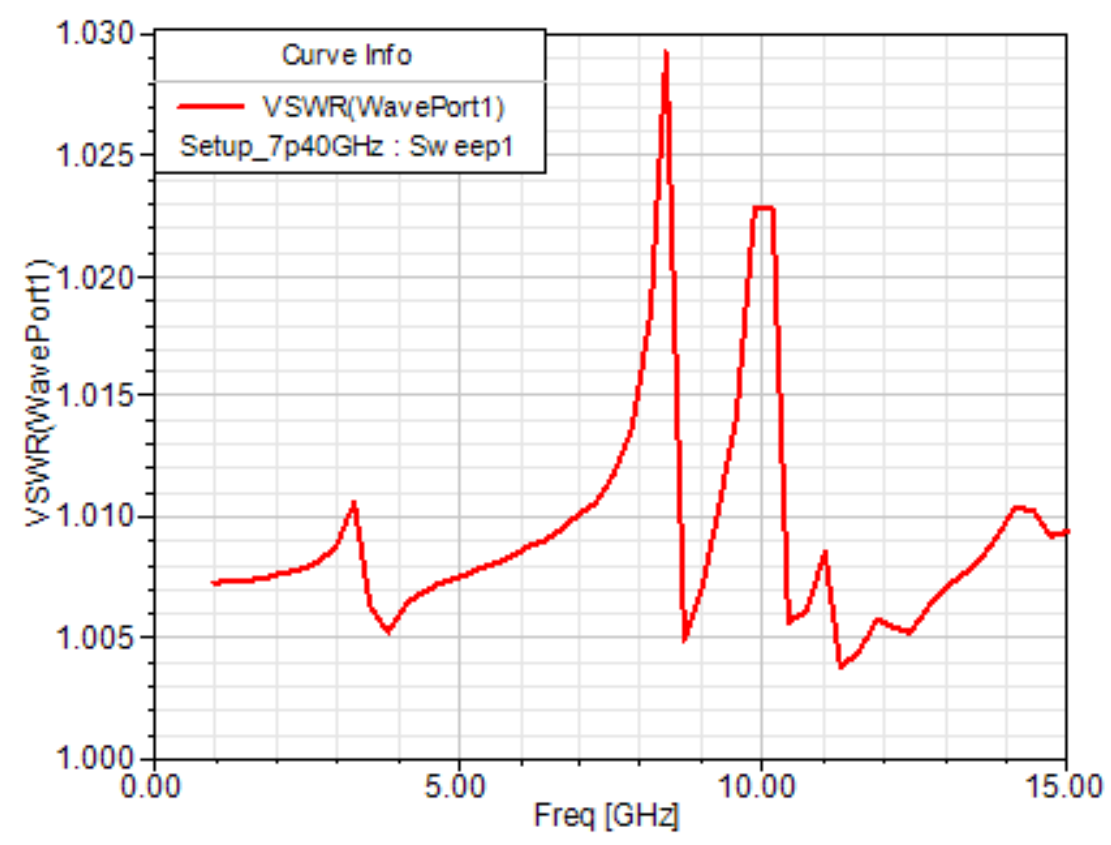

Fig. 5.17 VSWR when the antenna was simulated using an RT Durroid 5880 substrate for the second iteration

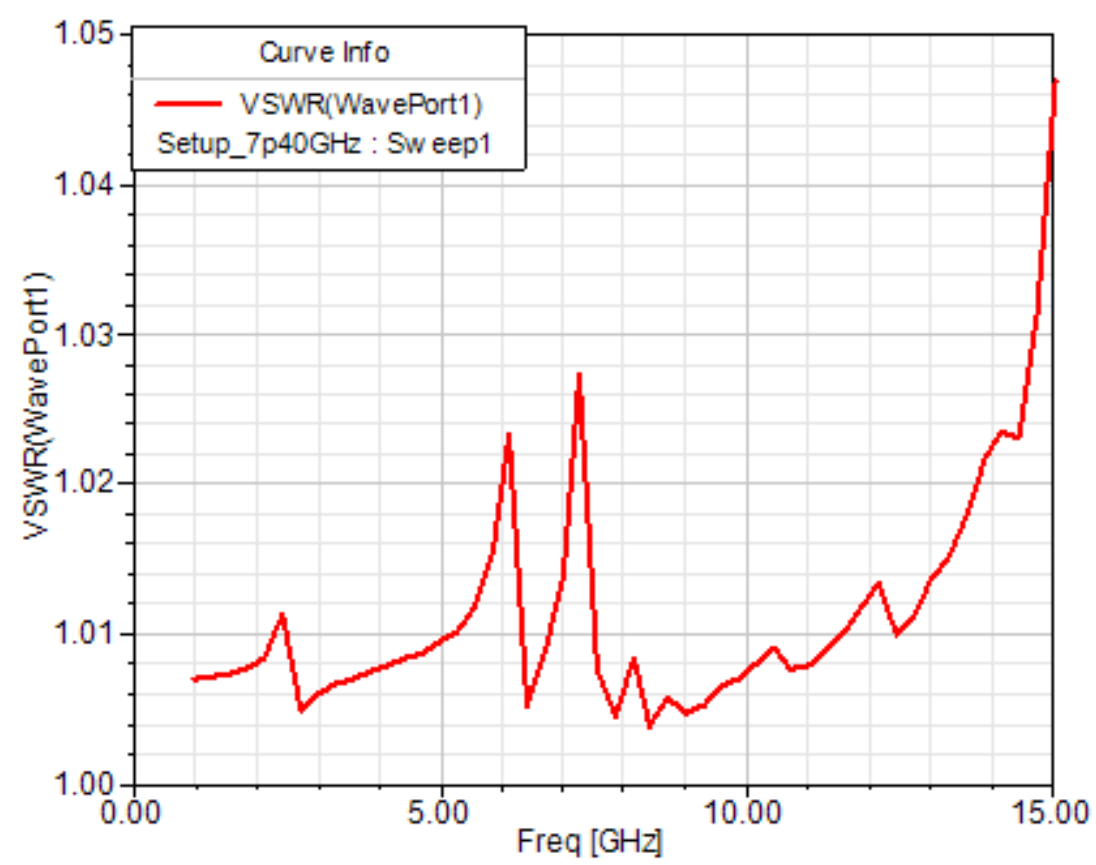

Fig. 5.18 VSWR when the antenna was simulated using an FR4 epoxy substrate for the second iteration 


\subsubsection{Data Analysis}

The RT Durroid 5880 material is considered to be a better substrate than the FR4 epoxy material. PCBs with an RT Durroid 5880 substrate have lesser capacitance losses, and are thus costlier than PCBs with an FR4 epoxy substrate. When the RRA was excited using a coaxial probe, it was found that it was in resonance throughout the entire frequency range from $1 \mathrm{GHz}$ to $15 \mathrm{GHz}$. The radiation characteristics showed that the antenna has a bidirectional radiation pattern for the second and the third iterations. The VSWR is also below 2 for the entire frequency range. When the results of the RRA are compared to the results of the RSGA, RPIFA and the RPISGFA, a major improvement in the performance is achieved.

\subsubsection{Simulation of the RRA with a slot feed}

In the third iteration, a slot of dimensions $4 \mathrm{~mm} \times 2 \mathrm{~mm}$ was attached to the outer ring of rectangles, and a face of the slot was excited as a waveport. In the second iteration, the thickness of the outer ring of rectangles was made zero, and a slot (of dimensions $8 \mathrm{~mm} \times 2 \mathrm{~mm}$ ) whose face was excited as a waveport, was attached to the inner ring of rectangles. The input return losses, VSWR and the radiation pattern, were compared when the antenna was simulated using an FR4 epoxy and an RT Durroid 5880 substrate for the second and the third iterations.

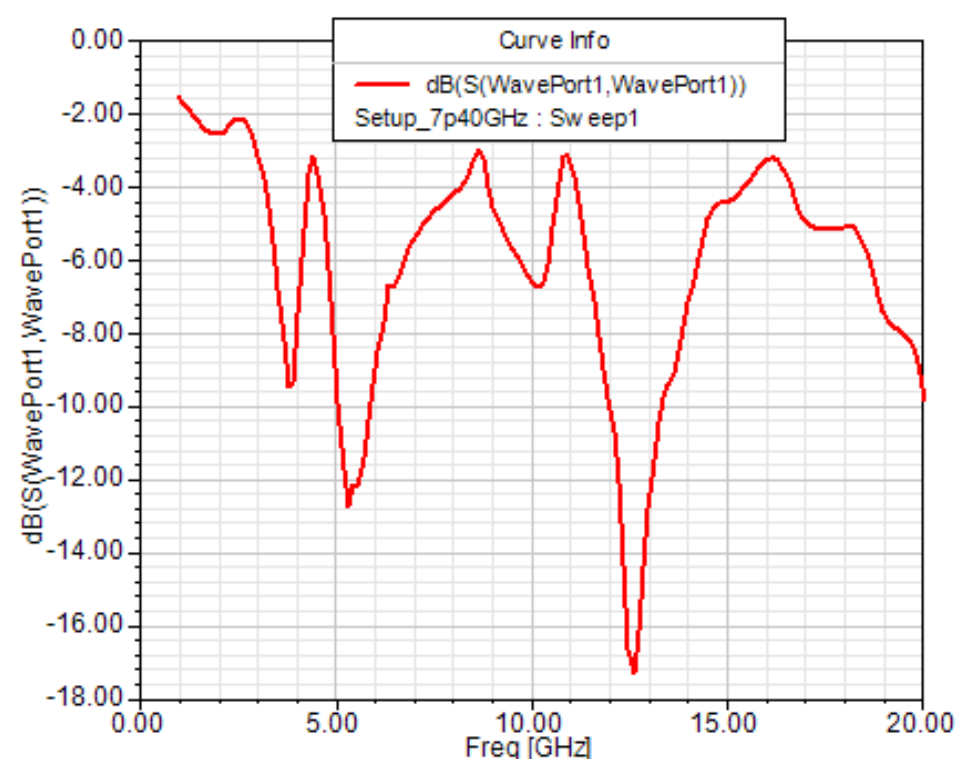

Fig. 5.19 Input Return Losses when the antenna was simulated using an RT Durroid 5880 substrate for the third iteration 


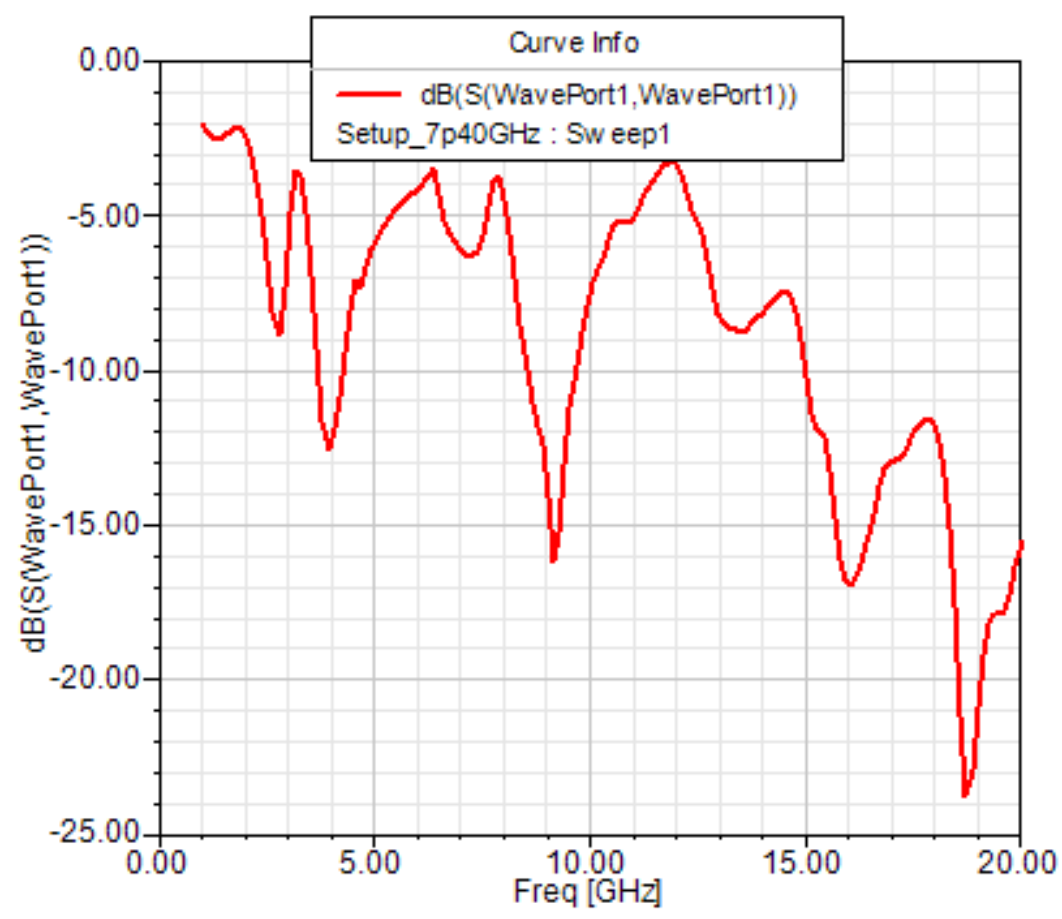

Fig. 5.20 Input Return Losses when the antenna was simulated using an FR4 epoxy substrate for the third iteration

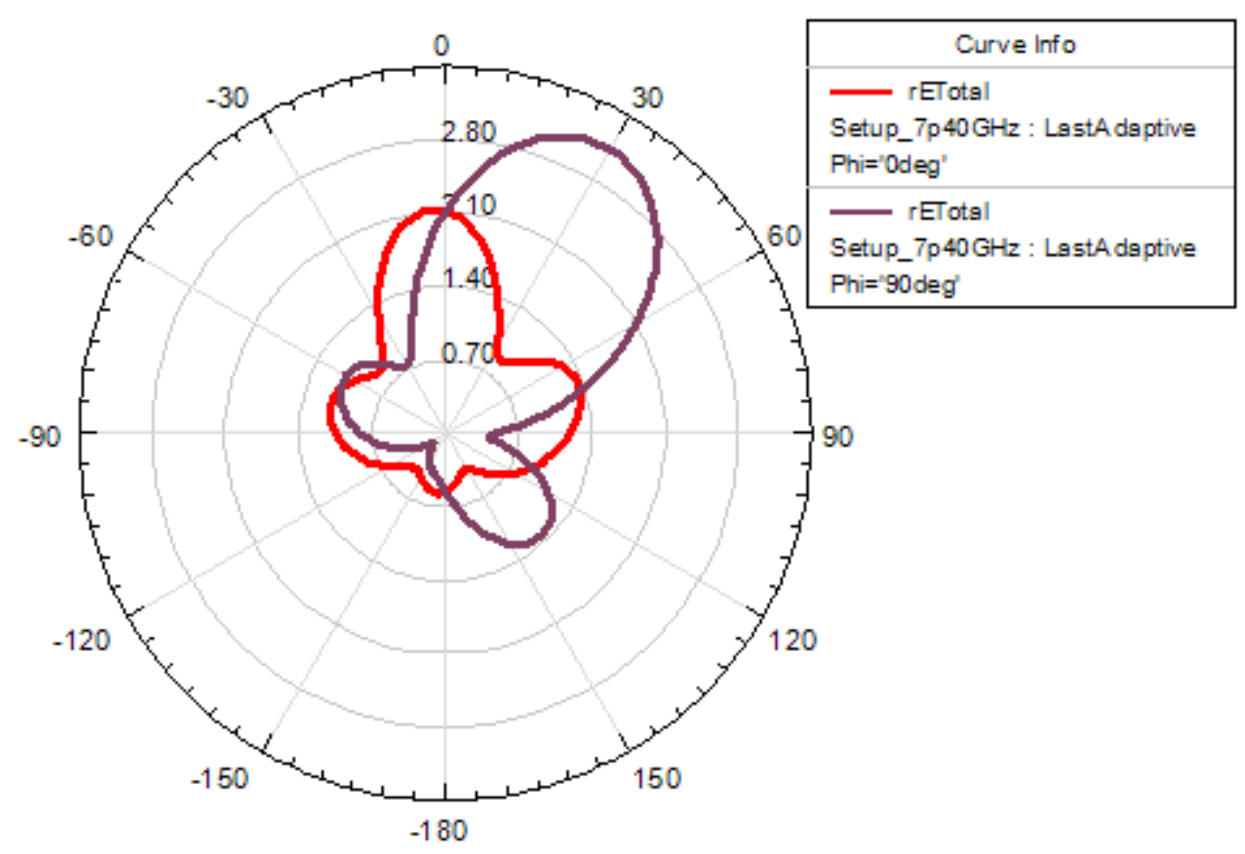

Fig. 5.21 Radiation Pattern when the antenna was simulated using an RT Durroid 5880 substrate for the third iteration 


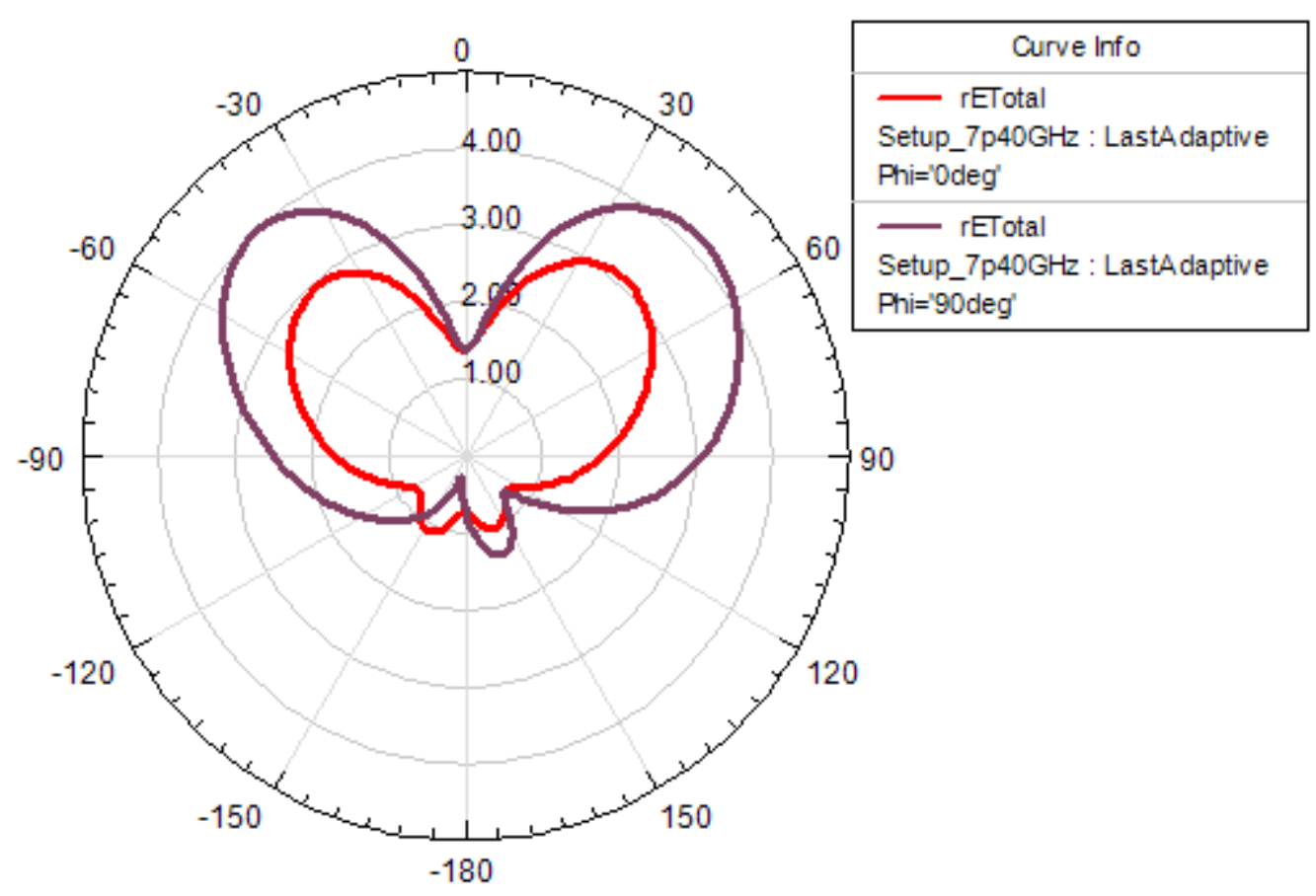

Fig. 5.22 Radiation Pattern when the antenna was simulated using an FR4 epoxy substrate for the third iteration

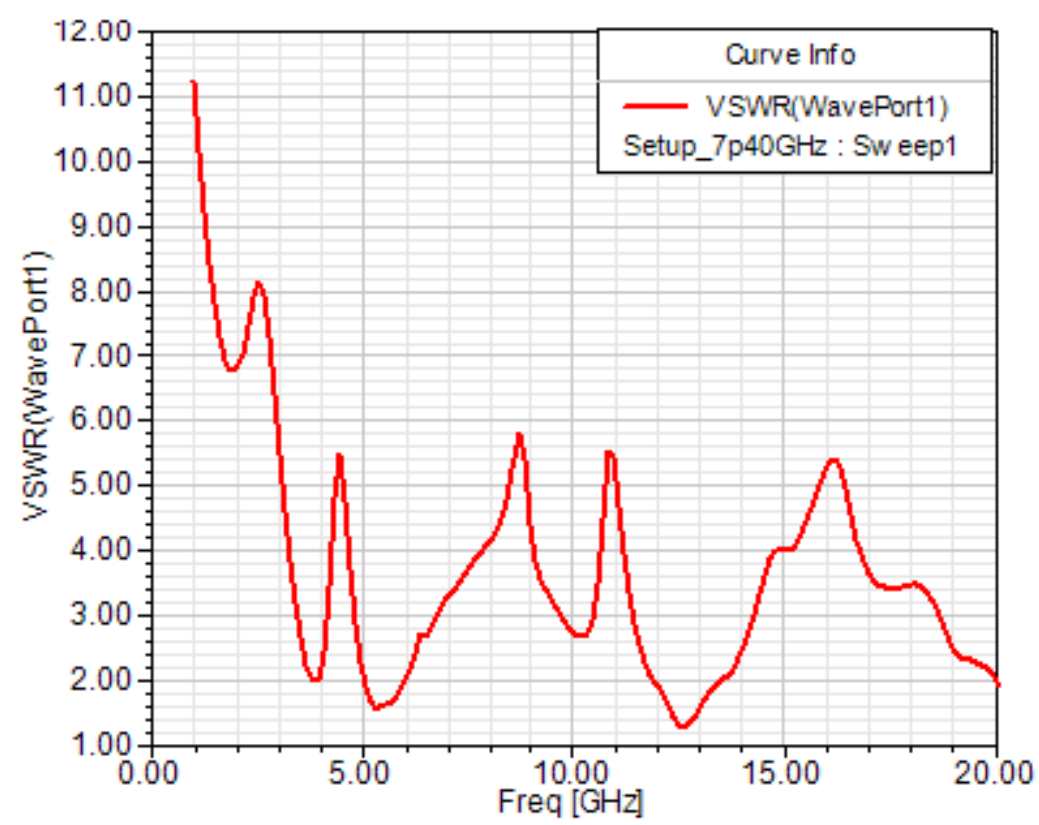

Fig. 5.23 VSWR when the antenna was simulated using an RT Durroid 5880 substrate for the third iteration 


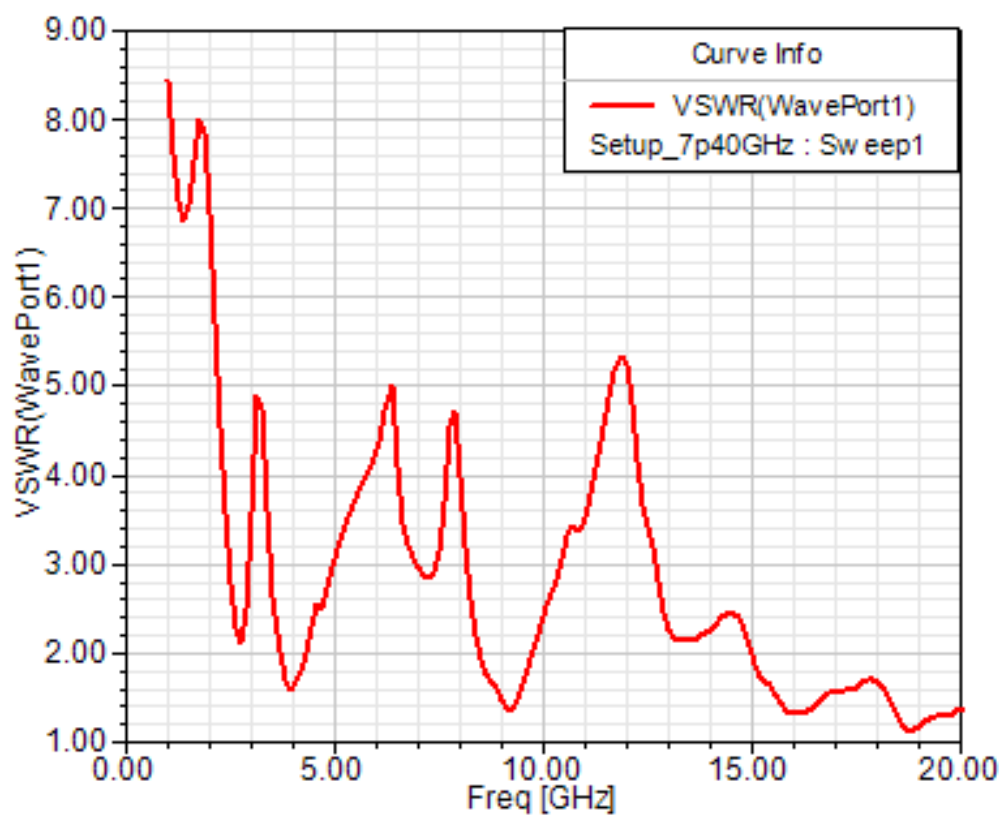

Fig. 5.24 VSWR when the antenna was simulated using an FR4 epoxy substrate for the third iteration

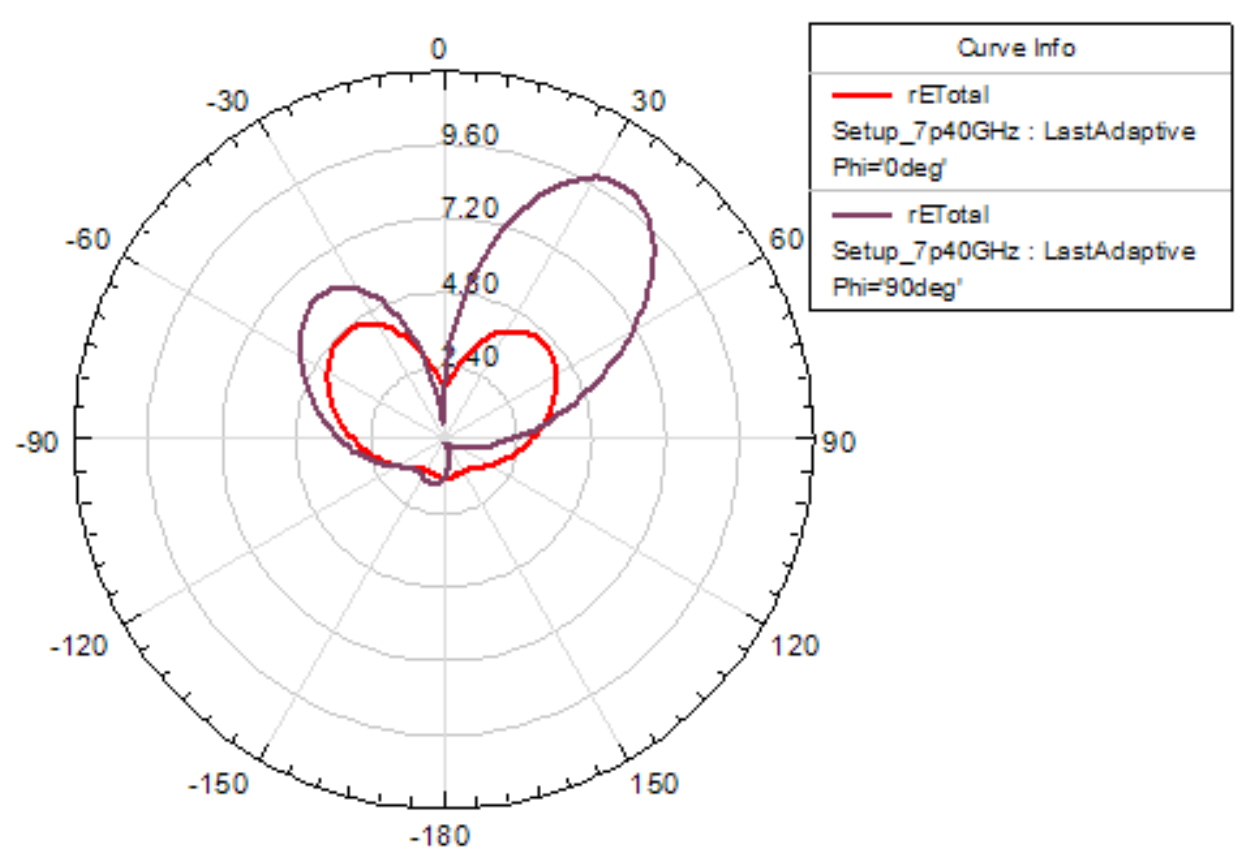

Fig. 5.25 Radiation Pattern when the antenna was simulated using an RT Durroid 5880 substrate for the second iteration 


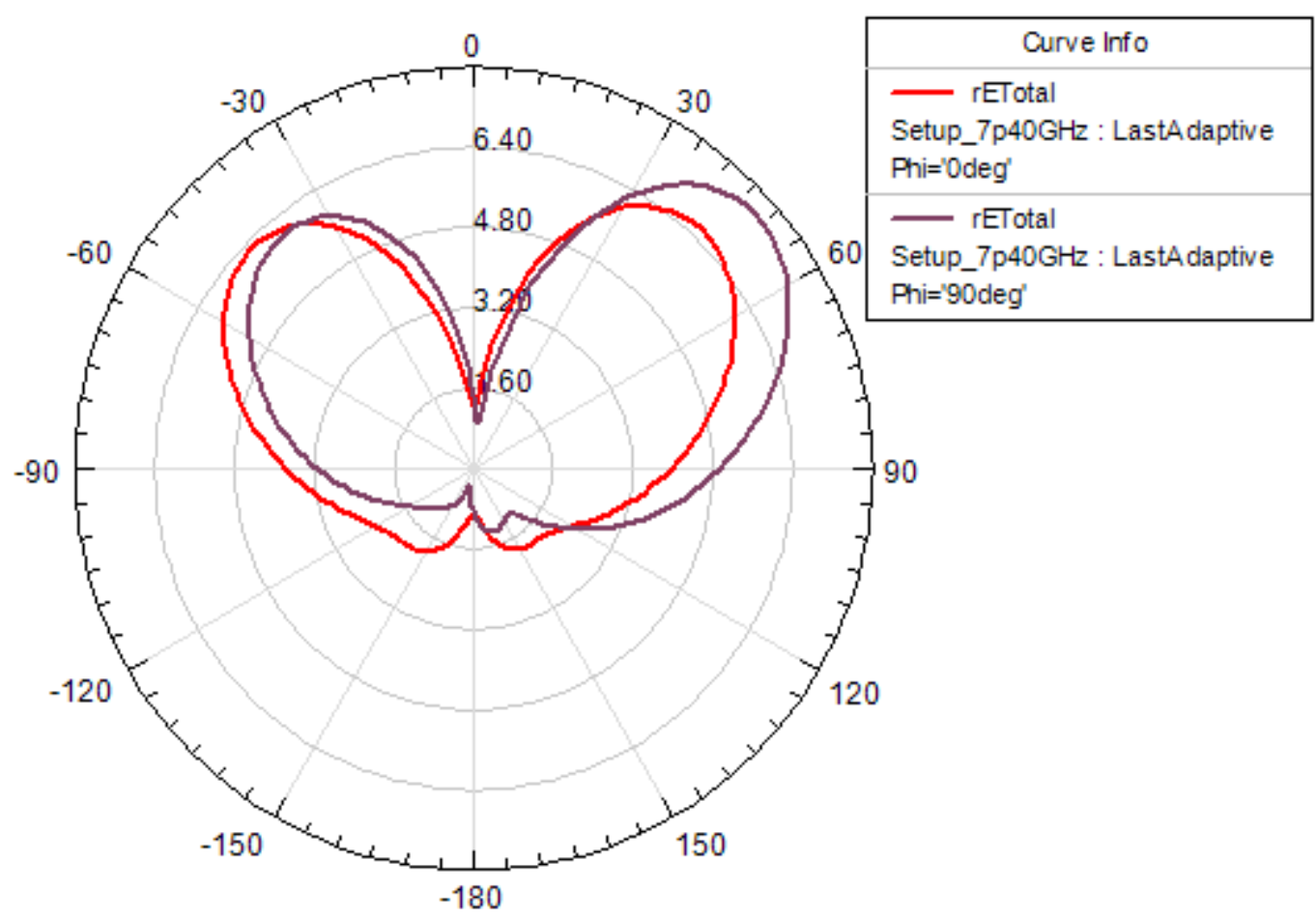

Fig. 5.26 Radiation Pattern when the antenna was simulated using an FR4 epoxy substrate for the second iteration

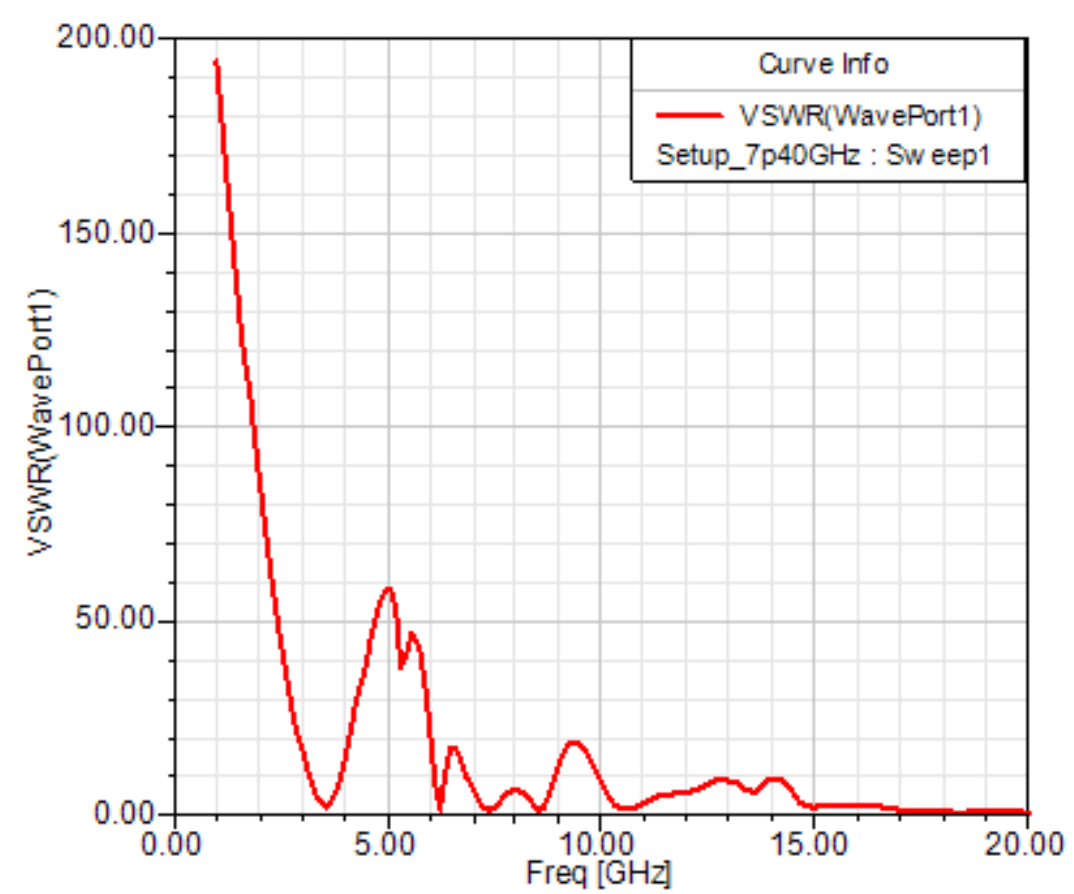

Fig. 5.27 VSWR when the antenna was simulated using an RT Durroid 5880 substrate for the second iteration 


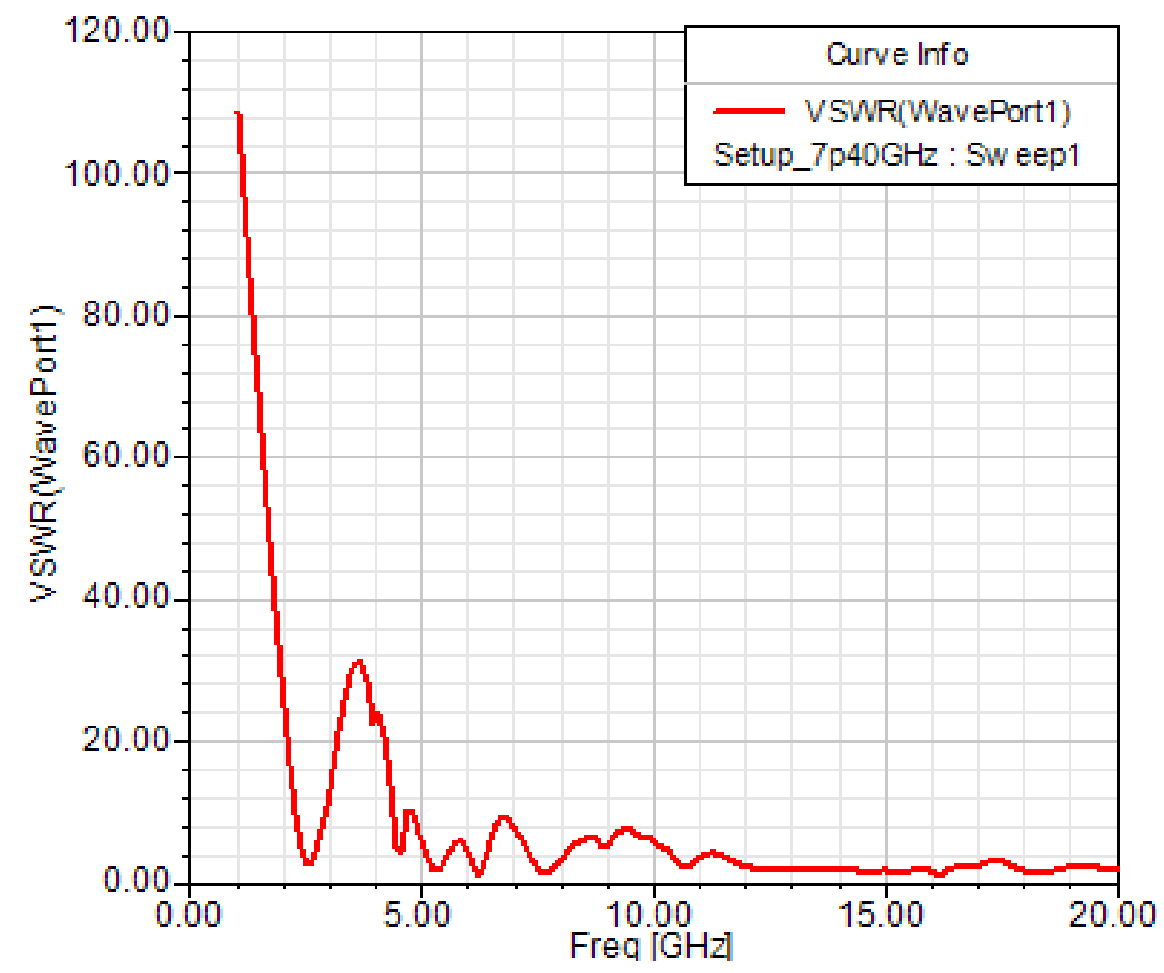

Fig. 5.28 VSWR when the antenna was simulated using an FR4 epoxy substrate for the second iteration

\subsubsection{Data Analysis}

The input return losses reveal two resonating frequencies when the antenna was simulated using an RT Durroid 5880 substrate, and four resonating frequencies when the antenna was simulated using an FR4 epoxy substrate. The radiation pattern characteristics show that the antenna has a bidirectional radiation pattern for the second and the third iterations. The value of VSWR falls below 2 when the antenna resonates.

\subsection{Lab Results}

Since simulation results varied depending on the method of excitation and substrate used, it was necessary to confirm that this antenna gives five resonating frequencies. This antenna was implemented on a PCB (FR4 epoxy substrate), and tested from $1 \mathrm{GHz}$ to $20 \mathrm{GHz}$ using an Agilent 8720 ES network analyzer, with a $3.5 \mathrm{~mm}$ test port cable with an SMA conductor. The input return losses and the VSWR plots (for the third iteration) from the network analyzer are presented and analyzed. 


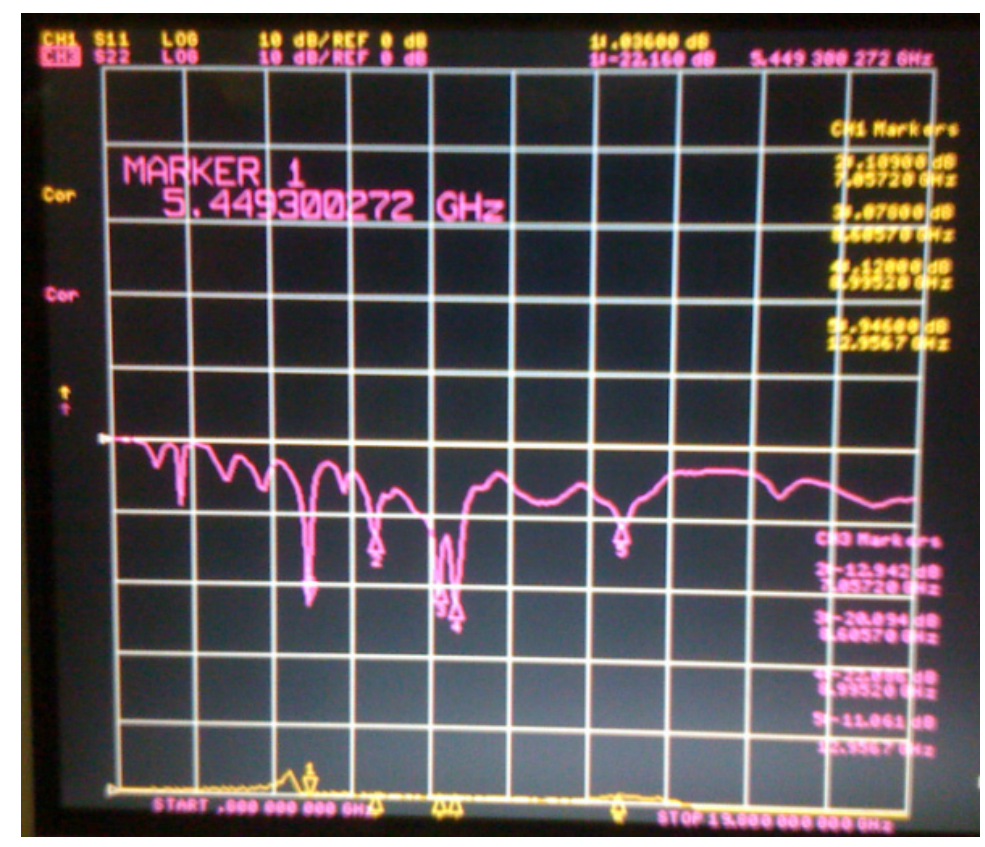

Fig. 5.29 Input Return Losses from the Network Analyser-1

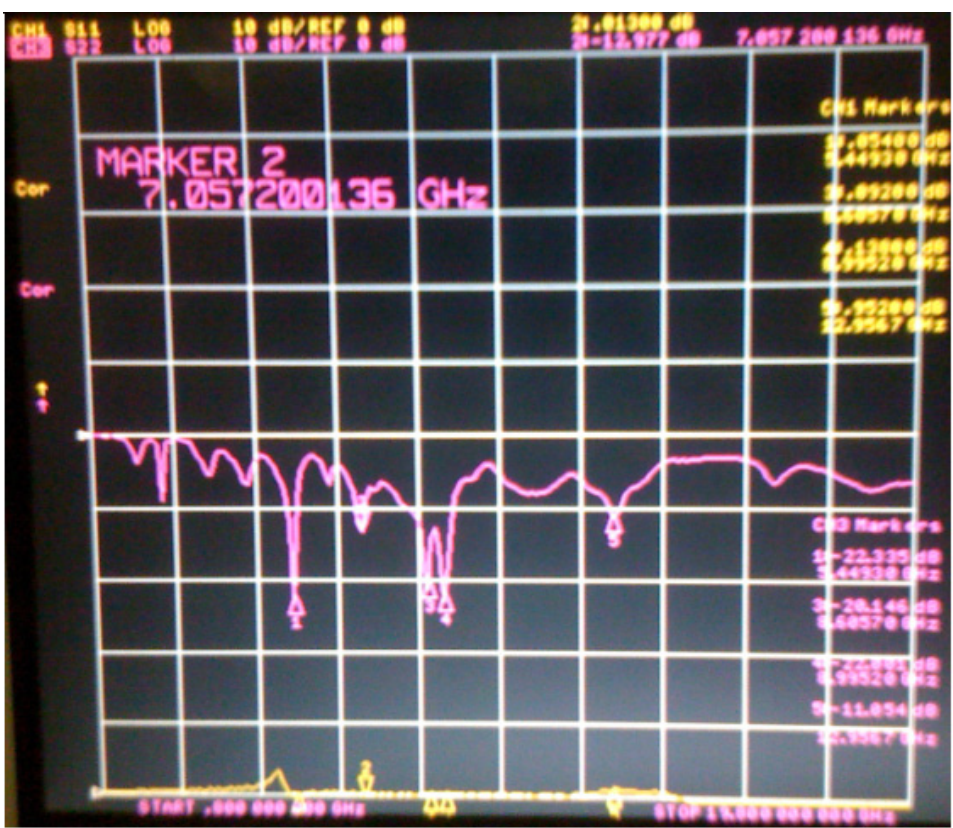

Fig. 5.30 Input Return Losses from the Network Analyser-2 


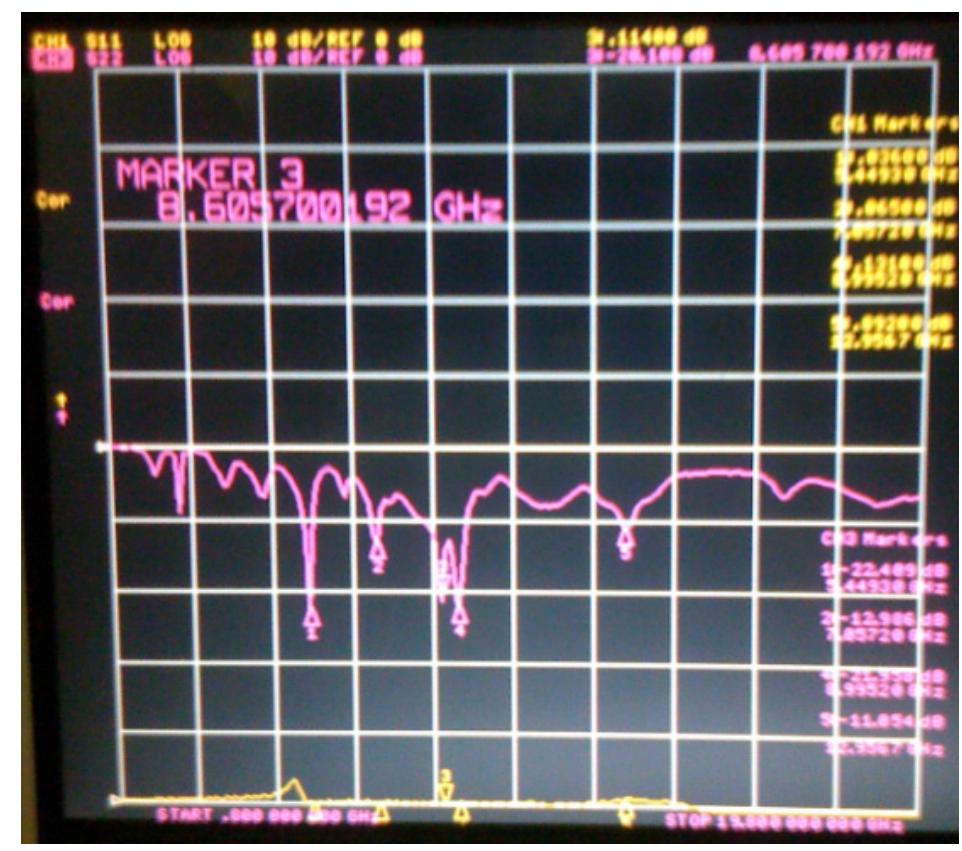

Fig. 5.31 Input Return Losses from the Network Analyser-3

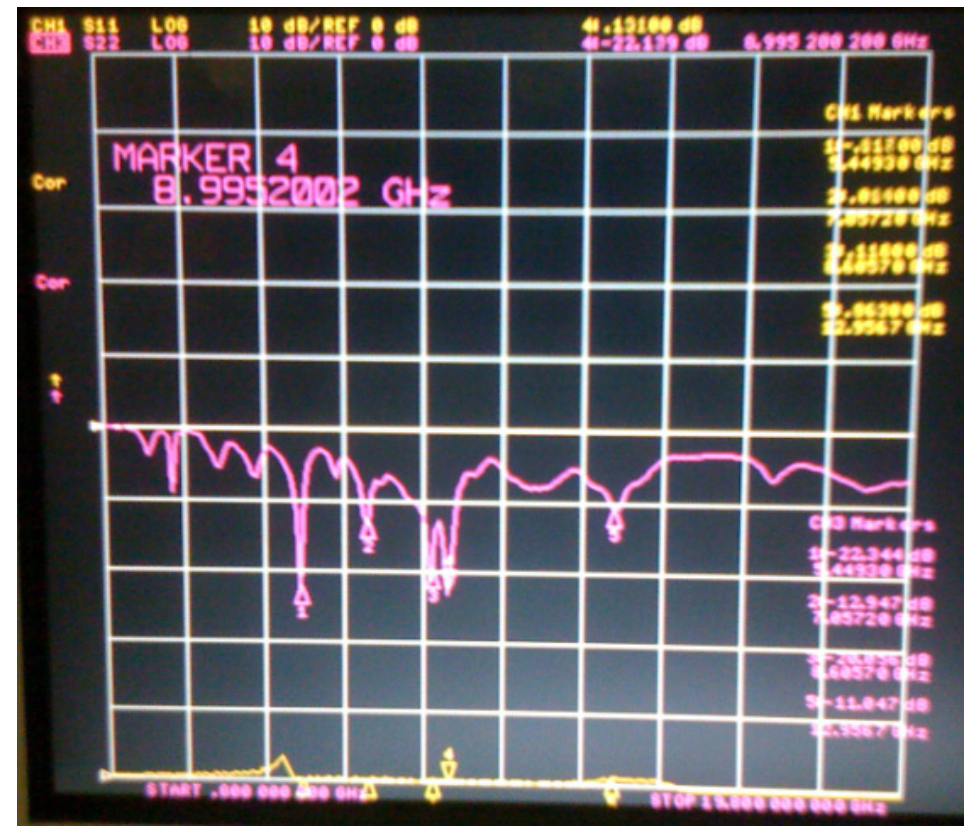

Fig. 5.32 Input Return Losses from the Network Analyser-4 


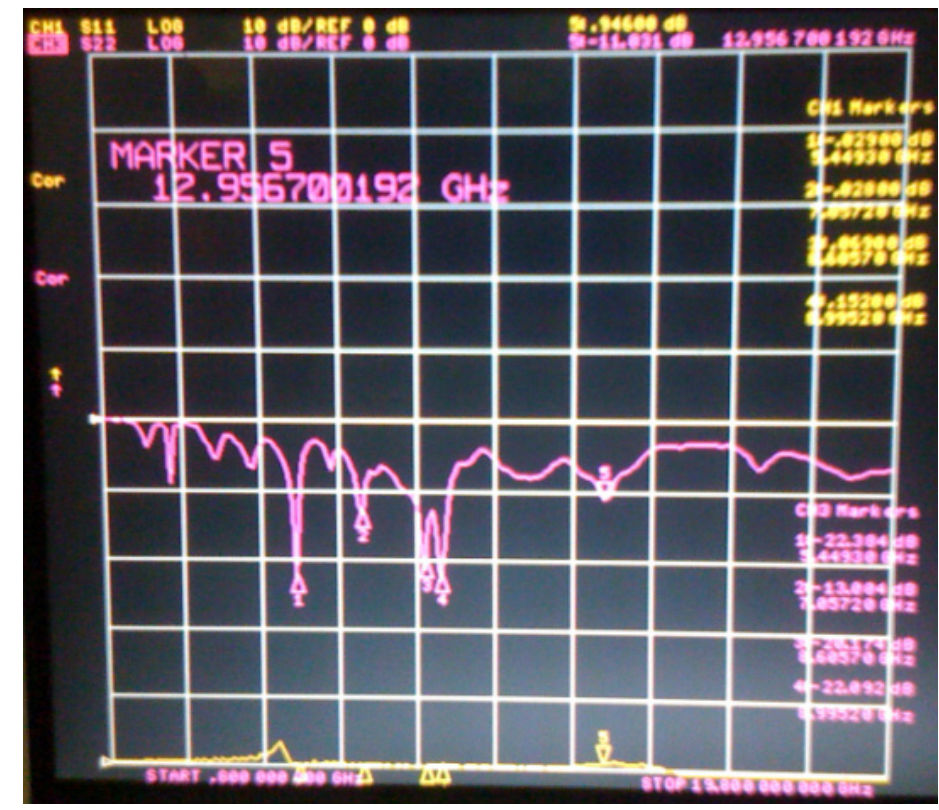

Fig. 5.33 Input Return Losses from the Network Analyser-5

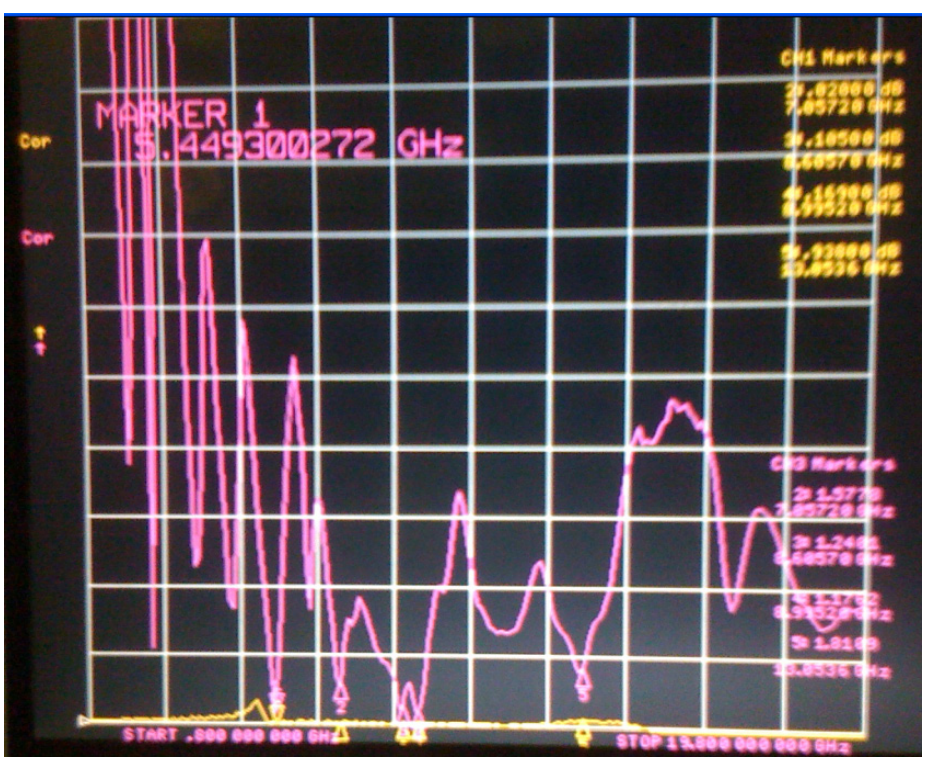

Fig. 5.34 VSWR from the Network Analyser 


\subsection{Summary}

The Rectangular Reconfigurable antenna can be analysed mathematically, and has multiple resonating frequencies. The design of the Rectangular Reconfigurable antenna is very simple, and more rectangular rings can be added to create more iterations. This antenna can be designed for the $\mathrm{MHz}$ and $\mathrm{GHz}$ frequency ranges. The antenna was found to be an ultrawideband antenna from our simulations, when it was excited using a coaxial probe feed. Analysing the results from the network analyser, it was found that this antenna definitely has 5 resonating frequencies, 3 of which are below $-20 \mathrm{~dB}$, and 2 of which are below $-10 \mathrm{~dB}$. This antenna can facilitate wireless powering, as it can be designed for any frequency range; and the eight switches can be used as eight separate feeder points to take power that is incident on the antenna. This antenna is small enough to be used in wireless applications, and the performance of these wireless applications can be enhanced by using this antenna. The fact that 5 resonating frequencies have been obtained is a significant contribution to the field of antenna research. 


\section{Chapter 6 \\ Conclusions and Future Work}

This dissertation explores the idea of enabling wireless powering in a concrete slab through enhanced reconfigurable antennas designs. The following are the major contributions made by this dissertation:

1. Simulations of a concrete slab in chapters 2 and 3 show that wireless powering in a concrete slab is possible, and can be done efficiently. The penetration of the surface of a concrete slab by a plane wave has not been done previously. Investigation of the penetration of the surface of a concrete slab, and the electric fields on the surface of the reconfigurable antennas inside the concrete slab, have shown that sensors can be powered wirelessly inside a concrete medium.

2. A novel planar inverted fractal antenna design has been shown in chapter 3 . The results from the RPISGFA have shown that the antenna has four resonating frequencies, which is a huge improvement when compared to the RSGA and the RPIFA.

3. Since the RPISGFA was made of two copper sheets above and below each other, a simpler antenna design with better performance was needed. The RRA has a simple design, and is a traditional microstrip antenna. Previous reconfigurable antenna designs have not been able to reveal the electric field, as well as the power radiated and directivity equations. Extensive mathematical analysis of the RRA is presented in this dissertation. Simulation results of the RRA with different substrates when it was excited using the coaxial probe and the slot are also presented.

4. The RRA was built on a double-sided PCB and tested using a network analyser to show that the lab results are matching the mathematical analysis. The results from the network analyser show much better performance than the RPISGFA, the RSGA and the RPIFA. 
5. Even if the RRA is not made reconfigurable with switches, the $2 \mathrm{~mm} \times 2 \mathrm{~mm}$ incremental lengths can still be used to convert the reconfigurable antenna into an ordinary antenna.

\subsection{Future Work}

The major focus of this dissertation involves four research areas:

1. Studying the existing reconfigurable antenna designs and identifying their short comings

2. Investigating the performance of reconfigurable antennas inside the concrete slabs

3. Creating a new reconfigurable planar inverted fractal antenna design

4. Creating a new reconfigurable microstrip antenna design and testing it using a network analyser

The investigation of reconfigurable antennas inside a concrete slab has been limited to simulation results. The new reconfigurable antennas presented in this dissertation, such as the RRA, and the RPISGFA, have to be fabricated and placed inside a concrete slab, and lab results have to be obtained. If an antenna is radiating more than $400 \mathrm{~mW}$ of power when it is close to the human brain, then it could cause brain cancer. Therefore, such experiments of reconfigurable antennas inside a concrete slab should be carried out in an anechoic chamber for safety purposes.

The RPISGFA and the RRA have to be fabricated and tested in an anechoic chamber. So far, only the input return losses and the radiation patterns of reconfigurable antennas have been tested in anechoic chambers. The RPISGFA and the RRA should be made to transmit a signal to another antenna, and their performance should be compared to an ordinary antenna. Reconfigurable antennas consist of multiple antennas that are turned on or off using switches. The coupling effects between the multiple radiating patches needs to be studied. Filters can be used to reduce these coupling effects. Effective filters need to be designed for the RPISGFA and the RRA if coupling exists between the radiating patches.Instead of switches, the RPISGFA and the RRA have to be simulated with diodes. If a positive external voltage is 
applied to diodes, then, at a certain voltage the diode is turned on. Similarly, at a certain negative potential the diode is turned off. These effects are popularly known as the forward bias and the reverse bias effects. These effects of a diode to external voltage allow them to be used as switches for reconfigurable antennas. The performance of the RPISGFA and RRA has to be tested using diodes in an anechoic chamber.

Micro Electro Mechanical Systems (MEMS) switches are also used in reconfigurable antennas. MEMS switches are fabricated using materials such as sapphire, quartz and barium chloride. Some MEMS switches also consist of cantilever beams. A voltage potential called the actuation voltage is used to turn the MEMS switch on. The actuation voltage depends on the design of MEMS switches, and it ranges from 2V to 90V. MEMS switches provide ultra-low losses, high isolation, and high linearity of relays. MEMS switches are usually used in military applications. The novel reconfigurable antennas shown in this dissertation, such as the RPISGFA and the RRA, have to be fabricated with MEMS switches. The MEMS switch fabricated RPISGFA and the RRA should be made to transmit a signal to another receiving antenna, and their performance should be compared to an ordinary antenna.

Multiple-Input and Multiple-Output (MIMO) systems consist of multiple antennas at both the transmitter and receiver to improve communication performance. So far, MIMO systems have only been developed with array antennas. MIMO systems need to be developed with reconfigurable antennas. The coupling effects of reconfigurable antennas in a MIMO environment need to be studied. 


\section{BIBLIOGRAPHY}

[1] Loizeau, S, Sibille, "A.Reconfigurable PIFA Antenna with Ultrawideband Tuning”, Antennas and propagation, 2007, EuCAP 11-16 Nov. 2007, pp 1-6, ISBN: 978-086341-842-6.

[2] N. Kingsley, D. E. Anagnostou, M. Tentzeris, and J. Papapolymerou, "Novel Design for MEMS Reconfigurable Sierpinski Antenna on a Flexible, Organic Substrate Without DC Bias Lines", IEEE/ASME Journal of MEMS.

[3] Punjala Shishir,"Wireless Powering of Sensors inside Concrete",International Conference on Advanced Technologies for Communication,Hanoi 2008.

[4] Punjala Shishir, Kia Makki "Rectangular Reconfigurable Antenna (RRA) with Ultra Wideband Tuning Ability", EUCAP 2009.

[5] Punjala Shishir,Kia Makki,"Wireless Powering of Sensors inside Concrete using a Reconfigurable Sierpinski Gasket Antenna”, EUCAP 2009.

[6] Punjala Shishir,Kia Makki, "Wireless Powering of Sensors inside Concrete using a Reconfigurable PIFA antenna", ANTEM/URSI 2009.

[7] L Sandrolini, U Reggiani and A Ogunsola,"Modeling the electrical properties of concrete for shielding effectiveness prediction", Journal of Physics and Applied Sciences, 2007.

[8] Shantanu A. Bhalerao, Abhishek V. Chaudhary, Raghavendra B. Deshmukh, Rajendra M. Patrikar, "Powering Wireless Sensor Nodes using Ambient RF Energy", 2006 IEEE International Conference on Systems, Man, and Cybernetics October 8-11, 2006, Taipei, Taiwan.

[9] Miaoudakis, A. Zacharopoulos, V. Stratakis, D. Antonidakis, E., "Self-powering wireless sensors in typical building environment", Globecom Workshops, 2007 IEEE, 26-30 Nov. 2007, page(s): 1-8, ISBN: 978-1-4244-2024-7.

[10] Soutsos M, Bungey J, Millard S, Shaw M and Patterson A,2001 NDT\&E Int.34419-25 ISSN 0963-8695.

[11] Shaari A, Millard S G and Bungey J H 2004 NDT\&E Int. 37237-242.

[12] Constantine A. Balanis, "Advanced Engineering Electromagnetics”, second edition, Wiley Publications, 1997. 
[13] Constantine A. Balanis, “Antenna Theory, Analysis and Design”, Wiley Publications, Third Edition, 1997.

[14] Fawwaz T. Ulaby, "Electromagnetics for Engineers", Pearson Education, 2005.

[15] Abdias Magalhäes Gomes, Juliana Oliveira Costa, Horácio Albertini, José Eduardo Aguiar, "Permeability of Concrete: A Study Intended for the "in situ" Valuation Using Portable Instruments and Traditional Techniques", Non-Destructive Testing in Civil Engineering, International Symposium (NDT-CE 2003).

[16] Kazimierz Siwiak and Yasaman Bahreini, "Radiowave Propagation and Antennas for Personal Communication, Third Edition, Artech House, 2007.

[17] Dennis Roddy, “Satellite Communications”, Fourth Edition, McGraw-Hill, 2007.

[18] Juan M. Rius, Jose Maria Gonzalez Arbesú, Jordi, Romeu, Eugenia Cabot, Michael Mattes, Juan R. Mosig, Rafael Gómez, Amelia Rubio, Mario Fernadez Pantoja, "Exploring the limits of Fractal Electrodynamics for the future Telecommunication Technologies", July 31 2003, IST-2001-33055

[19] Cetiner, B.A. Jofre, L. Chang, C.H. Qian, J.Y. Bachman, M. Li, G.P. De Flaviis, F. "Integrated MEM antenna system for wireless communications ", Dept. of Electr. \& Comput. Eng., California Univ., Irvine, CA, Microwave Symposium Digest, 2002 IEEE MTT-S International.

[20] Cetiner, B.A. Jafarkhani.H, Jiang-Yuan Qian, Hui Jae Yoo Grau, A. De Flaviis, F. "Multifunctional reconfigurable MEMS integrated antennas for adaptive MIMO systems", Center of Space Sci., Morehead State Univ., NC, USA, Communications Magazine, IEEE, 2004.

[21] Feldner,L.M.Nordquist, C.D. Christodoulou, C.G. "RF MEMS reconfigurable triangular patch antenna", Antennas and Propagation Society International Symposium, 2005 IEEE.

[22] B. N. Kim, S. O. Park, Y. S. Yoon, J. K. Oh, K. J. Lee, and G. Y. Koo, "Hexaband Planar Inverted-F Antenna With Novel Feed Structure for Wireless Terminals", IEEE Antennas and Wireless Propagation Letters, Vol. 6, pp. 66-69, 2007

[23] H. P.Chang et al.,"Low Cost RF MEMS Switches Fabricated on Microwave Laminate PCBs,” IEEE Elect. Device Lett., vol. 24, no.4, Apr. 2003, pp. 227-29.

[24] W. Arrighetti, P. De Cupis, G. Gerosa, "Circuit Models for the Sierpinski Gasket Antenna", physics/0510069, Computational Physics , Electronic Engineering Department, "La Sapienza" University of Rome, Italy, WMB0022-E . 
[25] C.C Liu, A.Hessel and J.Shmoys, "Performance of Probe-Fed Rectangular Microstrip Patch Element Phased Arrays", IEEE Trans. Antennas Propagat., Vol. AP-36, No. 11, pp. 1501-1509, November 1988.

[26] Sava V. Savov and Matti H. A. J. Herben, "Modal Transmission-Line Modeling of Propagation of Plane Radio waves through Multilayer Periodic Building Structures", IEEE Transactions on Antennas and Propagation, Vol. 51, No. 9, September 2003.

WilliamLee,"Mobile Cellular Telecommunications Analog and Digital Systems",Second Edition,McGraw-Hill International Editions, 1995.

[28] M.Kulkarni, "Microwave and Radar Engineering", Third Edition, Umesh Publications, 2003.

[29] S. Meninger, J.O. Mur-Miranda, R. Amirtharajah, A. Chandrakasan, J.H. Lang, "Vibration-to-electric energy conversion", IEEE Trans. VLSI Systems, " pp. 64-76, Vol. 9, Issue 1, Feb 2001.

[30] Seshagiri Krishnamoorthy, "Interference measurements and throughput analysis for 2.4 GHz wireless devices in hospital environments", MS Thesis, Dept. Elect. Eng., Virginia polytechnic and State Univ., Blacksburg, Virginia, 2003.

[31] Adam M. Alevy, "In-Building propagation measurements at 2.4 Ghz", in Wireless Symposium Feb. 2002.

[32] I.F. Akyildiz, Weilian Su, Y. Sankarasubramaniam, E. Cayirci, "A Survey on Sensor Networks", in IEEE Communications Magazine, pp.102-114, Vol. 40, Issue 8, Aug, 2002.

[33] J.A. Hagerty, et al, "Recycling ambient microwave energy with broad-band rectenna arrays", IEEE Trans. Microwave theory and techniques, pp.1014-1024, vol. 52, issue 3, Mar. 2004.

[34] F. Kocer, P.M. Walsh, M.P. Flynn, "An RF powered, wireless temperature sensor in quarter micron CMOS", in Proc. ofISCAS'04, pp. IV-876-879, Vol. 4, May 2004

[35] R.L. Li, G. DeJean, E. Tsai, E. Tentzeris, J. Laskar, "Novel; small folded shorted patch antennas", in IEEE Int. Symp. Antennas and Propagation Society, pp. 26-29, vol. 4, 1621 Jun 2002.

[36] D. E. Anagnostou et al., "Design, fabrication, and measurements of an RF-MEMS-based self-similar reconfigurable antenna," IEEE Trans. Antennas Propag., vol. 54, no. 2, pt. 1, pp. 422-432, Feb. 2006. 
[37] D.Werner and S.Ganguly, “An overview of fractal antenna engineering research,"IEEE AntennasPropag. Mag., vol. 45, no. 1, pp. 38-57, Feb. 2003.

[38] J. Anguera, C. Puente, C. Borja, and J. Soler, "Fractal-shaped antennas: A review," in Wiley Encyclopedia of RF and Microwave Engineering, vol. 2, K. Chang, Ed. New York: Wiley, 2005, pp. 1620-1635.

[39] C. Puente, J. Romeu, R. Pous, X. Garcia, and F. Benitez, "Fractal multiband antenna based on the Sierpinski gasket," Electron. Lett., vol. 32, no. 1, pp. 1-2, Jan. 1996.

[40] D. E. Anagnostou, G. Zheng, L. Feldner,M. T. Chryssomallis, J. C. Lyke,J. Papapolymerou, and C. G. Christodoulou, "Silicon-etched re-configurable self-similar antenna with RF-MEMS switches," in Proc. Antennas and Propagation Society Symp., vol. 2, Jun. 20-25, 2004, pp. 1804-1807.

[41] D.Culler, D. Estrin, M. Srivastava, "Overview of Sensor Networks", IEEE Computer Magazine, pp. 41-49, August 2004.

[42] Martinez, K.;Hart,J.K.;Ong, R.,"Environmental Sensor Networks”,IEEE Computer Magazine, Vol. 37, Issue 8, Page(s):50 - 56, Aug. 2004.

[43] Andreas Willing, Kirsten Matheus and Adam Woliszd, "Wireless Technology in Industrial Networks,"Proceedings of IEEE, vol. 93, no. 6, pp. 1130-1151, June 2005.

[44] Humirel HM1500LF humidity sensor datasheet available online at http://humirel.com/

[45] IEEE Standard Definitions of Terms of Antennas,IEEEStd 145-1993,SH16279,March 28,1993 .

[46] IEEE Standard Definitions of Terms for Radio Wave Propagation, IEEE Std 2111990, SH13904,1990.

[47] I. Lier and K. R. Jakobsen, "Rectangular Microstrip Patch Antenna with Infinite and Finite Ground-Plane Dimensions”, IEEE Trans. Antennas Propagat., Vol. AP-31, No. 6, pp. 978-984, Nov 1983.

[48] R. W. Lee, K. F. Lee and J. Bonbinchak, "Characteristics of a Two-Layer Electromagnetically Coupled Rectangular Patch Antenna," Electronic Letters, Vol.23, pp. 1070-1072, September 1987.

[49] G. Gronau and I. Wolff, "Aperture-Coupling of a Rectangular Microstrip Resonator", Electronic Letters, Vol. 22, pp. 554-556, May 1986. 
[50] J. R. Mosig and F. E. Gardiol, "General Integral Equation Formulation for Microstrip Antennas and Scatterers,” Proc. Inst. Elect. Eng., pt. H, Vol. 132, pp. 424-432, 1985.

[51] C.C.Liu, A. Hessel and J. Shmoys, "Performance of Probe-Fed Rectangular Microstrip Patch Element Phased Arrays," IEEE Trans. Antennas Propagat., Vol. AP-36, No. 11, pp. 1501-1509, Nov 1988.

[52] Philip Felber "Fractal Antennas" A Literature Study, Illinois Institute of Technology, Dec 2000.

[53] K.D Prasad “Antenna \& Wave Propagation” Third Edition, 1995.

[54] F.R. Terman,” Electronic Radio and Engineering”. MacGraw-Hill.

[55] P.Lorrain, D. R. Corson, and F. Lorrain. "Electromagnetic fields and waves: including electric circuits”. Freeman, New York, 1988.

[56] Pozar, David M. "Microwave Engineering", Third Edition (Intl. Ed.); John Wiley \& Sons, 2005, Inc.; pp 170-174. ISBN 0-471-44878-8.

[57] J Choma \& WK Chen. "Feedback networks: theory and circuit applications". Singapore: World Scientific. Chapter 3, p. 225 ff, 2007. ISBN 981-02-2770-1.

[58] Hutchinson,Chuck, “The ARRL Handbook for Radio Amateurs 2001"Newington,CT: ARRL — the national association for Amateur Radio.pp. g. 20.2. ISBN 0-87259-186-7.

[60] Elkamchouchi. H, Nasr. M.A, "3D-Fractal Rectangular Koch Dipole and Hilbert Dipole Antennas", Microwave and Millimeter Wave Technology, 2007. ICMMT '07, 18-21 April 2007, Page(s):1-4, Digital Object Identifier 10.1109/ICMMT.2007.381294.

[61] Douglas H. Werner and Raj Mittra,"Frontiers in Electromagnetics," New York,IEEE Press, 2000.

[62] John Gianvittorio,"Fractals, MEMS, and FSS Electromagnetic Device: Miniaturization and Multiple Resonances," PhD dissertation, University of California, Los Angeles, 2003.

[63] J. M. Gonzalez, S. Blanch, J. Romeu, "The Hilbert Curve as a Small Self- Resonant Monopole From a Practical Point of View", Microwave Optical Technology Letters., vol. 39, no. 1, October 2001.

[64] Iulian Rosu, "PIFA - Planar Inverted F Antenna", YO3DAC / VA3IUL, RF Technical Articles. 
[65] Leland et al. "On the self-similar nature of Ethernet traffic", IEEE/ACM Transactions on Networking, Volume 2, Issue 1, February 1994.

[66] I.J. Bahl amd P. Bhartia, "Microstrip Antennas”, Artech House, Dedham, MA, 1980.

[67] G. M. Rebeiz, "RF MEMS theory, design, and technology," Hoboken, NJ: John Wiley \& Sons, 2003.

[68] B.A. Cetiner, H. Jafarkhani, J.Y. Qian, H.J. Yoo, A. Grau, and F. DeFlaviis, "Multifunctional reconfigurable MEMS integrated antennas for adaptive MIMO systems," IEEE Commun. Mag., vol.42, no.12, pp.62-70, 2004.

[69] B.A. Cetiner,J.Y.Qian,G.P.Li and F.De Flaviis, "A reconfigurable spiral antenna for adaptive MIMO systems" EURASIP Journal on Wireless Commun. and Networking, pp 382-389, 2005.

[70] Jennifer T. Bernhard"Reconfigurable Antennas" Synthesis Lecture on Antennas, Morgan and Caypool Publishers, 2007 
February 10, 1985

July 2006

December 2007
Born, Hyderabad, Andhra Pradesh India

B.E Electronics and Communications Engineering Osmania University

Hyderabad, Andhra Pradesh, India.

M.S Telecommunications and Networking

Florida International University

Miami, FL, USA.

\section{PUBLICATIONS AND PRESENTATIONS}

Punjala Shishir,"Wireless Powering of Sensors inside Concrete", International Conference on Advanced Technologies for Communication, Hanoi 2008.

Punjala Shishir, Kia Makki "Rectangular Reconfigurable Antenna (RRA) with Ultra Wideband Tuning Ability", EUCAP 2009.

Punjala Shishir,Kia Makki,"Wireless Powering of Sensors inside Concrete using a Reconfigurable Sierpinski Gasket Antenna”, EUCAP 2009.

Punjala Shishir,Kia Makki, "Wireless Powering of Sensors inside Concrete using a Reconfigurable PIFA antenna", ANTEM/URSI 2009. 